Article

\title{
New Evidence of the Marginal Predictive Content of Small and Large Jumps in the Cross-Section
}

\author{
Bo Yu, Bruce Mizrach and Norman R. Swanson * \\ Department of Economics, School of Arts and Sciences, Rutgers University, 75 Hamilton Street, \\ New Brunswick, NJ 08901, USA; byu@econ.rutgers.edu (B.Y.); mizrach@econ.rutgers.edu (B.M.) \\ * Correspondence: nswanson@economics.rutgers.edu
}

Received: 19 February 2020; Accepted: 2 May 2020; Published: 19 May 2020

\begin{abstract}
We investigate the marginal predictive content of small versus large jump variation, when forecasting one-week-ahead cross-sectional equity returns, building on Bollerslev et al. (2020). We find that sorting on signed small jump variation leads to greater value-weighted return differentials between stocks in our highest- and lowest-quintile portfolios (i.e., high-low spreads) than when either signed total jump or signed large jump variation is sorted on. It is shown that the benefit of signed small jump variation investing is driven by stock selection within an industry, rather than industry bets. Investors prefer stocks with a high probability of having positive jumps, but they also tend to overweight safer industries. Also, consistent with the findings in Scaillet et al. (2018), upside (downside) jump variation negatively (positively) predicts future returns. However, signed (large/small/total) jump variation has stronger predictive power than both upside and downside jump variation. One reason large and small (signed) jump variation have differing marginal predictive contents is that the predictive content of signed large jump variation is negligible when controlling for either signed total jump variation or realized skewness. By contrast, signed small jump variation has unique information for predicting future returns, even when controlling for these variables. By analyzing earnings announcement surprises, we find that large jumps are closely associated with "big" news. However, while such news-related information is embedded in large jump variation, the information is generally short-lived, and dissipates too quickly to provide marginal predictive content for subsequent weekly returns. Finally, we find that small jumps are more likely to be diversified away than large jumps and tend to be more closely associated with idiosyncratic risks. This indicates that small jumps are more likely to be driven by liquidity conditions and trading activity.
\end{abstract}

Keywords: forecasting; integrated volatility; high-frequency data; jumps; realized skewness; cross-sectional stock returns; signed jump variation

\section{Introduction}

Theoretical models of the risk-return relationship anticipate that volatility should be priced, and that investors should demand higher expected returns for more volatile assets. However, ex-ante risk measures are not directly observable, and must be estimated (see e.g., Rossi and Timmermann (2015)). Given the necessity of estimating volatility, various risk estimators have been used in the empirical literature studying the strength and sign of the risk-return relationship. Unfortunately, the evidence from the literature is mixed, in the sense that researchers have found both negative and positive relationships between return and volatility. One possible reason for these surprisingly contradictory findings is that the risk-return relationship is nonlinear. Examples of papers pursuing this hypothesis include Campbell and Vuolteenaho (2004), who incorporate different factor betas based on good and bad news about cash flows and discount rates; and Woodward and Anderson (2009) who find that bull and bear market betas differ substantially across 
most industries. This research has helped to spawn the "smart-beta" approach to factor investing. ${ }^{1}$ In related research, Feunou et al. (2013) model the effects of volatility in positive and negative return states separately. They define so-called disappointment aversion preferences, and show that investors should demand a higher return for downside variability. These authors find empirical support for their model in the U.S. and several foreign markets using a bi-normal GARCH process to estimate volatility.

In this paper, we focus on the importance of jumps in volatility for understanding the risk-return relationship. We do this by assessing the marginal predictive content of small versus large jump variation, when forecasting one-week-ahead cross-sectional equity returns. We also examine earnings announcements as well as carry out various Fama-MacBeth type regressions to uncover the linkages between (small and large) jumps and news. Finally, we examine the importance of control variates, including skewness and other firm characteristics, when undertaking to disentangle the relative importance of small, large, positive, and negative jumps for the dynamic evolution of firm specific returns. Much of the empirical research that explores the importance of jumps in this context focuses on the estimation of continuous and jump variation components using nonparametric realized measures constructed with high-frequency financial data. A key paper in this area is Bollerslev et al. (2020), who examine the relationship between signed jumps and future stock returns in the cross-section. They document that signed jump variation, which captures the asymmetric impact of upside and downside jump risk, is a good predictor of future returns, particularly for small and illiquid stocks. ${ }^{2}$ We add to this literature by decomposing jump variation into signed small and large components and evaluating the importance of these elements in the cross-section of stock returns. We use the cross-section of individual stocks because aggregate index returns may mask small jump effects on return predictability. Indeed, many studies document that aggregation may diversify away idiosyncratic small jumps in the cross-section (see e.g., Ait-Sahalia and Jacod (2012); Duong and Swanson (2015)).

The motivation for our paper can be traced back to Yan (2011) and Jiang and Yao (2013), who show that large, infrequent jumps are priced in the cross-section of returns. Feunou et al. (2018) take the decomposition used by these authors one step further, and model jumps in the realized semi-variances of market returns. They construct a new measure of the variance risk premium, and find a strong positive premium for downside risk. Fang et al. (2017) find a similar result for Chinese market returns. In a related line of research, various authors study the information content in upside and downside jump variation. For example, Guo et al. (2015) document that at the market level, a negative jump component in realized volatility predicts an increase in future equity premia. Bollerslev et al. (2015) identify both left and right jump tail risks under the risk-neutral measure. They find that the left jump tail risk is an appropriate proxy for market fear. Additionally, they find that including a variance risk premium together with jump tail risk measures as predictors significantly improves market return forecasts. Finally, they show that jump risk helps explain the high-low book-to-market and winners-versus-losers portfolio returns.

Building on the above literature, we decompose jump variation into four distinct components depending on both the direction (upward and downward) and magnitude (small and large) of the jumps. ${ }^{3}$ Specifically, we decompose individual stock jump (semi) variances into small and large components, using high-frequency intraday data. We then investigate the relationship

1 In 2017, Morningstar reported that this approach to investing has attracted over one trillion dollars in assets (see e.g., Jennifer Thompson, Financial Times, 27 December 2017).

2 In a related paper, Duong and Swanson (2015) construct both small and large jump measures based on some fixed truncation levels. They exploit the risk predictability of different jump measures using both index data and Dow 30 stocks and find that small jump variation has more volatility predictability than large jump variation.

3 The methods that we implement to separate jump variation use recent advances in financial econometrics due to Andersen et al. (2007, 2003), Jacod (2008), Mancini (2009), Barndorff-Nielsen et al. (2010), Todorov and Tauchen (2010), Ait-Sahalia and Jacod (2012), and Patton and Sheppard (2015). Most importantly, the reader is referred to Ait-Sahalia and Jacod (2012), who survey the methods used in this paper. 
between these various jump measures and future returns, using single- and double-sorted stock portfolios, and Fama-MacBeth cross-sectional regression analysis. The reason that we decompose jump semi-variances into small and large components is that this decomposition allows us to explore the possibility that they contain different information relevant to investing and return predictability. As Maheu and McCurdy (2004) note, large jumps may reflect important individual stock and market news announcements. Smaller jumps (or continuous variations) may result from liquidity and strategic trading.

Of note is that in our setup, the number of large jumps is always finite, regardless of jump activity. However, there may exist infinitely many small jumps. This framework is surveyed in Ait-Sahalia and Jacod (2012), where cutoff (truncation) methods used for decomposing total jump variation into large and small jump components are outlined. We follow their approach when constructing large and small jump variation measures. Summarizing, we construct jump variation measures by estimating the difference between quadratic variation and power variation, as well as by decomposing quadratic variation into jump and non-jump components using cutoffs. The cutoff (truncation) values that we use are related to those discussed in Li et al. (2017), and in Ait-Sahalia and Jacod (2012). However, we are not interested in jump detection (or jump testing), per-se, in discussed in Bollerslev et al. (2008), but are instead interested in the estimation and analysis of jump variation measures. For this reason, our methodology most closely mirrors that discussed in Ait-Sahalia and Jacod (2012). In contexts where jump testing is of interest, many approaches are available. For example, one may rely on the conservative approach discussed in Bollerslev et al. (2008), or the false discovery control and spurious jump detection approach discussed in Bajgrowicz et al. (2016) and Scaillet et al. (2018). For further discussion of testing, the reader is referred to Lee and Mykland (2008, 2012), Barras et al. (2010), Bajgrowicz and Scaillet (2012), Christensen et al. (2014), and the references cited therein. ${ }^{4}$

Our key findings can be summarized as follows. First, we find that both small and large upside (downside) jump variation negatively (positively) predicts subsequent weekly returns. However, portfolios sorted using signed total jump variation are associated with increased average returns and risk-adjusted alphas for high-low portfolios, relative to the cases where upside or downside jump variation is sorted on. This finding is in accord with the findings of Bollerslev et al. (2020).

Our second finding involves the case where jump variation is further decomposed into "small" and "large" components. In this case, sorting on signed small jump variation leads to value-weighted high-low portfolios with greater average returns and alphas than when either signed total jump or signed large jump variation is sorted on. Indeed, when the truncation parameter used to differentiate small from large jumps is based on a 5 standard-deviation (i.e., $\alpha=5$ ) cutoff, we find that average return spreads are $10 \%$ higher when signed small jump variation is sorted on rather than signed total jump variation. Moreover, these average return spreads are statistically significant in both cases. However, average return spreads are not significantly different from zero when signed large jump variation is sorted on. Indeed, including large jump variation is actually detrimental to predictive accuracy, as average returns and alphas for high-low portfolios actually decline relative to the case where total variation is instead used in our prediction experiments. These results suggest that there may be a "jump-threshold", beyond which large jump variation contains no marginal predictive ability,

4 As noted by the editor, an important issue in the context of the estimation of jump variation components is the assessment of the importance of the cutoff methods used in this paper when disentangling small and large jump variations. Our approach to this issue in the sequel is to assess the robustness of our empirical findings to the use of various different cutoffs. However, Monte Carlo simulation may shed further light on the issue. In undertaking Monte Carlo simulations, one must carefully simulate both infinite-activity and finite-activity jump processes, consider various portfolios of simulated assets, and calibrate DGPs using models fitted to panels of high-frequency asset prices and returns. Although this topic is important, it is beyond the scope of the current paper, and is left to future research. 
relative to that contained in small jump variation. ${ }^{5}$ In summary, we find that large jump variation has little to no marginal predictive content, beyond a certain threshold. ${ }^{6}$ Indeed, when the threshold is judiciously selected, one can actually improve predictive performance in our experiments, leading to increased high-low portfolio average returns and alphas, when sorting portfolios based on small jump variation rather than total jump variation.

To understand why small jumps matter in our analysis, note that Scaillet et al. (2018) document that jumps are frequent events and that jump dynamics are not consistent with the compound Poisson processes with constant intensity. In addition, many recent studies find that infinite-activity Levy jump specifications (e.g., Variance Gamma and Normal Inverse Gaussian return jumps) can improve the goodness of fit and option pricing performance of models (e.g., see Yang and Kanniainen (2016)). These results point to the possible importance of small jumps, which may have infinite numbers in a finite time interval. Though more frequent than large jumps, note that we estimate daily jump variation measures and aggregate them into weekly measures for forecasting future weekly returns. Thus, it is not individual small jumps that necessarily matter, but the overall contribution of small jumps to total variation, in the cross-section. Additionally, we show (see below discussion) that small jump variation is more closely associated with idiosyncratic risk than is large jump variation. If idiosyncratic volatility matters (see Ang et al. (2006, 2009)), so do small jumps, at least to some extent. Indeed, our results partially help explain the idiosyncratic volatility puzzle (investors prefer positive skewness/asymmetry, so they will accept lower returns for stocks with higher probabilities of having positive jumps).

Third, industry double-sorts indicate that the benefit of small signed jump variation investing is driven by stock selection within an industry, rather than industry bets. Investors prefer stocks with a high probability of having positive jumps, but they also tend to overweight safer industries.

Fourth, the reason small and large (signed) jump variation measures have differing marginal predictive content for returns is associated with the importance of realized skewness as a control variable in our experiments. Specifically, we find that in double-sorted portfolios, the content of signed large jump variation is negligible when controlling for either signed total jump variation or realized skewness. By contrast, signed small jump variation has unique information for predicting future returns, even when controlling for total jump variation or realized skewness. This finding is consistent with the results from a series of Fama-MacBeth regressions, in which we control for multiple firm characteristics and risk measures.

Finally, small and large jump variation measures are driven by different economic factors and contain different information for predicting future returns. For example, large jumps are closely associated with "big" news. In particular, large earning announcement surprises increase both the magnitude and occurrence of large jumps. While such news-related information is embedded in large jump variation, the information is generally short-lived, and dissipates too quickly to provide marginal predictive content for subsequent weekly returns. This is consistent with our finding that filtering out signed small jump variation (which we know to be useful) from signed total jump variation results

5 When equal-weighted portfolios are instead examined, sorting on total jump variation yields higher average returns and alphas than when sorting on small or large jump variation. However, deeper inspection of our tabulated results in this case reveals that average returns associated with large jump variation sorts are much smaller-around 1/2 of the magnitude of those associated with small and total jump variation sorts. Also, the magnitude of average returns associated with small jump variation sorts is much closer (within $10 \%$ ) to the average returns associated with total jump variation sorts, when our truncation parameter uses a 5 standard-deviation cutoff instead of a 4 standard-deviation cutoff. This suggests that the jump-threshold differs depending upon portfolio type, and indicates that our findings based on equal-weighted portfolios are largely in accord with the findings elucidated above.

6 This result is not in contradiction with the extant literature on the importance of large jumps. This is because all our conclusions are for the cross-section (our paper is the first one that investigates the return predictability of large/small jump variation measured in the cross-section). It is true that large jumps have been shown to have return/variance predictability for individual stocks/portfolios. But in the cross-section, we do not observe such return predictability. In this cross-section, this is simply because large jumps are rare, so that they provide little (or at least infrequent) information for future weekly returns, in the cross-section. 
in increased predictive ability, relative to the case where only signed total jump variation is used in return forecasting, particularly for big firms. Additionally, this finding is interesting, given that the comparison of aggregated and weighted jump variation measures indicates that small jump variation captures idiosyncratic risk. More specifically, we find that small jumps are more likely to be diversified away than large jumps, thus tend to be more closely associated with idiosyncratic risks, and are therefore more likely to be driven by liquidity conditions and trading activity. ${ }^{7}$

In closing, it is worth stressing that the magnitudes of price movements depend on the magnitude of news (if they are related to news), and on the underlying process describing price evolution, and thus movements may manifest in the form of large/small jumps and/or price drifts. Needless to say, the process associated with how firms and markets digest information is critical to understanding and quantifying what sorts of price movements ensue. For any given news, responses may differ in terms of upside or downside price drifts, or large/small jumps. In this sense, both small and large jumps may matter, as shown in our empirical results, in the cross-section. Also, note that idiosyncratic volatility, one of the most studied asset pricing anomalies, is one of the most successful predictors for stock returns in the cross-section (see Gu et al. (2019)). As we show that small jumps are more likely to be associated with idiosyncratic risk, and since signed small jump variation has a negative relationship with future stock returns, our results help explain the idiosyncratic volatility puzzle. This is because of agent's preference for lottery-like returns (i.e., investors will accept lower returns for stocks with high probabilities of having positive jumps).

The rest of this paper is organized as follows. In Section 2 we discuss the model setup and define the jump risk measures that we use. Section 3 contains a discussion of the data used in our empirical analysis, and highlights key summary statistics taken from our dataset. Section 4 presents our main empirical findings, including discussions of results based on single portfolio sorts, double-sorts, cumulative return and Sharpe ratio analysis, firm-level Fama-MacBeth regressions, and finally, jumps and news announcements. Section 5 concludes.

\section{Model Setup and Estimation Methodology}

Following Aït-Sahalia and Jacod (2012), assume that the log price, $X_{t}$, of a security follows an Itô semimartingale, formally defined as:

$$
X_{t}=X_{0}+\int_{0}^{t} b_{s} d s+\int_{0}^{t} \sigma_{s} d W_{s}+\int_{0}^{t} \int_{\{|x| \leq \gamma\}} x(\mu-v)(d s, d x)+\int_{0}^{t} \int_{\{|x| \geq \gamma\}} x \mu(d s, d x),
$$

where $b$ and $\sigma$ denote the drift and diffusive volatility processes, respectively; $W$ is a standard Brownian motion; $\mu$ is a random positive measure with its compensator $v$; and $\gamma$ is the (arbitrary) cutoff level (threshold) used to distinguish between small and large jumps. As pointed out in Ait-Sahalia and Jacod (2012), the continuous part of this model (i.e., the $\int_{0}^{t} \sigma_{s} d W_{s}$ term) captures the normal hedgeable risk of the asset. The large jumps part of the model (i.e., the $\int_{0}^{t} \int_{\{|x| \geq \gamma\}} x \mu(d s, d x)$ term) might capture big news-related events such as default risk, and the small jumps part of the model (i.e., the $\int_{0}^{t} \int_{\{|x| \leq \gamma\}} x(\mu-v)(d s, d x)$ term) might arise due to other information flows, as discussed below. For cases where jumps are summable (e.g., when jumps have finite activity, so that

7 This result is consistent with the finding of Amaya et al. (2015) that preference for positive asymmetry (skewness) may partially explain the idiosyncratic volatility puzzle, especially for small firms. 
$\sum_{s \leq t} \Delta X_{s}<\infty$, for all $t$ ), then the size of a jump at time $s$ is defined as $\Delta X_{s}=X_{s}-X_{s-} .{ }^{8}$ In this context, the "true" price of risk is often defined by the quadratic variation, $Q V_{t}$, of the process $X_{t}$. Specifically,

$$
Q V_{t}=\int_{0}^{t} \sigma_{s}^{2} d s+\sum_{s \leq t} \Delta X_{s}^{2}
$$

where the variation of the continuous component (i.e., the integrated volatility) is given by $I V_{t}=\int_{0}^{t} \sigma_{s}^{2} d s$, and the variation of the price jump component is given by $Q J_{t}=\sum_{s \leq t} \Delta X_{s}^{2}$.

In the sequel, intraday stock returns are assumed to be observed over equally spaced time intervals in a given day, where the sampling interval is denoted by $\Delta_{n}$, and the number of intraday observations is $n$. Thus, the intraday log-return over the $i$ th interval is defined as

$$
r_{i, t}=X_{i \Delta_{n}, t}-X_{(i-1) \Delta_{n}, t}
$$

It is well known that when the sampling interval goes to zero, the realized volatility, $R V_{t}$, which is calculated by summing up all successive intraday squared returns, converges to $Q V_{t}$, as $n \rightarrow \infty$, where

$$
R V_{t}=\sum_{i=1}^{n} r_{i, t}^{2} \rightarrow{ }_{u} Q V_{t}=I V_{t}+Q J_{t}
$$

where $\rightarrow{ }_{u}$ denotes convergence in probability, uniformly in time.

To separate jump variation from integrated volatility, Andersen et al. (2007) show that the jump and continuous components of realized variance can be constructed as:

$$
R V J_{t}=\max \left(R V_{t}-\widehat{I V}_{t}, 0\right)
$$

and

$$
R V C_{t}=R V_{t}-R V J_{t},
$$

respectively, where $\widehat{I V}_{t}$ is an estimator of $\int_{0}^{t} \sigma_{s}^{2} d s$. Following Barndorff-Nielsen and Shephard (2004) and Barndorff-Nielsen et al. (2006), we use tripower variation to estimate the integrated volatility. In particular, define

$$
\widehat{I V}_{t}=V_{\frac{2}{3}, \frac{2}{3}, \frac{2}{3}} \mu_{\frac{2}{3}}^{-3}
$$

where $\mu_{q}=E\left(|Z|^{q}\right)$ is the $q$ th absolute moment of the standard normal distribution, and

$$
V_{m_{1}, m_{2}, \ldots m_{k}}=\sum_{i=k}^{n}\left|r_{i, t}\right|^{m_{1}}\left|r_{i-1, t}\right|^{m_{2}} \ldots\left|r_{i-k+1, t}\right|^{m_{k}},
$$

where $m_{1}, m_{2} \ldots m_{k}$ are positive, such that $\sum_{1}^{k} m_{i}=q$.

Now, again following Aït-Sahalia and Jacod (2012), we separate total jump variation into small and large variation measures, using various truncation levels, $\gamma$ (see Duong and Swanson $(2011,2015)$ for additional details). In particular, define realized small and large jump variation as follows:

$$
R V L J_{\gamma, t}=\min \left(R V J_{t}, \sum_{i=1}^{n} r_{i, t}^{2} I_{\left\{\left|r_{i, t}\right| \geq \gamma\right\}}\right)
$$

and

$$
R V S J_{\gamma, t}=R V J_{t}-R V L J_{\gamma, t}
$$

8 A jump process has finite activity when it makes a finite number of jumps, almost surely, in each finite time interval, otherwise it is said to have infinite activity. 
respectively, where $I(\cdot)$ denotes the indicator function, which equals one if the absolute return is larger than the truncation level, and is otherwise equal to zero. We are also interested in upside and downside variation measures associated with positive and negative returns. Following Barndorff-Nielsen et al. (2010), we construct realized semi-variances, defined as: $R S_{t}^{+}=\sum_{i=1}^{n} r_{i, t}^{2} I_{\left\{r_{i, t}>0\right\}}, R S_{t}^{-}=\sum_{i=1}^{n} r_{i, t}^{2} I_{\left\{r_{i, t}<0\right\}}$, and $R V_{t}=R S_{t}^{+}+R S_{t}^{-}$. These authors show that the above upside and downside semi-variances $\left(R S_{t}^{+}\right.$and $R S_{t}^{-}$, respectively) each converge to the sum of one half of the integrated volatility and the corresponding signed jump variation. Specifically,

$$
R S_{t}^{+} \rightarrow_{u} \frac{1}{2} \int_{0}^{t} \sigma_{s}^{2} d s+\sum_{s \leq t} \Delta X_{s}^{2} I_{\left\{\Delta X_{s}>0\right\}}
$$

and

$$
R S_{t}^{-} \rightarrow_{u} \frac{1}{2} \int_{0}^{t} \sigma_{s}^{2} d s+\sum_{s \leq t} \Delta X_{s}^{2} I_{\left\{\Delta X_{s}<0\right\}}
$$

We construct upside and downside jump variation as follows:

$$
R V J P_{t}=\max \left(R S_{t}^{+}-\frac{1}{2} \widehat{I V}_{t}, 0\right)
$$

and

$$
R V J N_{t}=\max \left(R S_{t}^{-}-\frac{1}{2} \widehat{I V}_{t}, 0\right)
$$

In addition, signed jump variation can be calculated as the difference between these upside and downside jump measures, as follows:

$$
S R V J_{t}=R V J P_{t}-R V J N_{t}
$$

This measure captures asymmetry in upside and downside jump variation.

In our analysis, we decompose the above upside and downside jump variation into small and large components, again using the cutoff (truncation level) discussed in Aitt-Sahalia and Jacod (2012). For further related discussion, see Mancini (2009), Duong and Swanson (2015), and Li et al. (2017). In particular, upside and downside large jump variations based on $\gamma$ are defined as follows:

$$
R V L J P_{\gamma, t}=\min \left(R V J P_{t}, \sum_{i=1}^{n} r_{i, t}^{2} I_{\left\{r_{i, t}>\gamma\right\}}\right)
$$

The corresponding downside measure is defined as follows:

$$
R V L J N_{\gamma, t}=\min \left(R V J N_{t}, \sum_{i=1}^{n} r_{i, t}^{2} I_{\left\{r_{i, t}<-\gamma\right\}}\right) .
$$

In all of our analyses, we construct $\gamma$ by estimating $\alpha \sqrt{\frac{1}{t} \widehat{I V}_{t}^{(i)}} \Delta_{n}^{0.49}$, thus accounting for the time-varying diffusive spot volatility of different stocks in the cross-section. ${ }^{9}$ In the sequel, we consider

9 As an example of how our cutoff level compares with others used in the literature, consider Li et al. (2017). These authors use bipower variation as the fixed value for $\widehat{I V}_{t}^{(i)}$. We instead use bipower variation as the initial value for the integrated volatility $\widehat{I V}_{t}^{(0)}$, say, and $\widehat{I V}_{t}^{(i)}$ is estimated using truncated bipower variation with threshold $\gamma^{(i-1)}$, say, where $\gamma^{(i-1)}$ is fixed only when $\left|\widehat{I V}_{t}^{(i)}-\widehat{I V}_{t}^{(i-1)}\right|$ is smaller than $5 \% \times \widehat{I V}_{t}^{(i-1)}$. 
three values for $\gamma$, say $\gamma^{1}$ (with $\left.\alpha=4\right), \gamma^{2}$ (with $\alpha=5$ ), and $\gamma^{3}$ (with $\alpha=6$ ). Our signed large jump variation (i.e., large jump asymmetry) measures are defined as follows:

$$
S R V L J_{t}=R V L J P_{t}-R V L J N_{t}
$$

Our corresponding small jump variation measures are defined as the difference between total and large jump variation. Specifically,

$$
R V S J P_{t}=R V J P_{t}-R V L J P_{t}
$$

and

$$
R V S J N_{t}=R V J N_{t}-R V L J N_{t} .
$$

Additionally, signed small jump variation is defined as:

$$
S R V S J_{t}=R V S J P_{t}-R V S J N_{t}
$$

To analyze the predictability of various jump measures in the cross-section, we normalize each of the jump variation measures discussed above by total realized variation.

An alternative to our approach for calculating the upside and downside jump variation measures in (1) and (2) is to use truncated realized variation (TRV) instead of tripower variation as a consistent estimator of integrated volatility, where $T R V_{t}=\sum_{i=1}^{n} r_{i, t}^{2} I_{\left\{\left|r_{i, t}\right| \leq \alpha_{n}\right\}} \rightarrow_{u} I V_{t}=\int_{0}^{t} \sigma_{s}^{2} d s$. Upside and downside jump variation can then be calculated using:

$$
R V J P_{t}=R S_{t}^{+}-\sum_{i=1}^{n} r_{i, t}^{2} I_{\left\{0<r_{i, t} \leq \alpha_{n}\right\}}
$$

and

$$
R V J N_{t}=R S_{t}^{-}-\sum_{i=1}^{n} r_{i, t}^{2} I_{\left\{-\alpha_{n} \leq r_{i, t}<0\right\}}
$$

where $\alpha_{n}$ is the truncation level. Please note that this truncation level is a generic "jump detection" truncation level, whereas $\gamma$ is a truncation level used to disentangle large and small jump variations from total jump variation. ${ }^{10}$ Our empirical findings based on the use of (10) and (11) to define $R V J P_{t}$ and $R V J N_{t}$ are qualitatively the same as those reported in Section 4 based on the use of (1) and (2).

To measure skewness and kurtosis, we construct higher order realized return moments. Following Amaya et al. (2015), standardized daily skewness is defined as:

$$
R S K_{t}=\frac{\sqrt{n} \sum_{i=1}^{n} r_{i, t}^{3}}{R V_{t}^{\frac{3}{2}}},
$$

and normalized daily realized kurtosis is defined as:

$$
R K T_{t}=\frac{n \sum_{i=1}^{n} r_{i, t}^{4}}{R V_{t}^{2}}
$$

Finally, it should be noted that we follow Amaya et al. (2015) and Bollerslev et al. (2020), and conduct our cross-sectional analysis at the weekly frequency. In particular, on each Tuesday, we compute the following weekly realized measures: $R V_{t}^{W}=\left(\frac{252}{5} \sum_{i=0}^{4} R V_{t-i}\right)^{1 / 2}$ and $R M_{t}^{W}=\frac{1}{5}\left(\sum_{i=0}^{4} R M_{t-i}\right)$, where $R V_{t}$ is defined above, and where $R M_{t}$ denotes any of the realized

10 Here, the threshold, $\alpha_{n}=3 \sqrt{\frac{1}{t} \widehat{I V}_{t}^{(i)}} \Delta_{n}^{0.49}$, is estimated using the same procedure as in footnote 9. 
measures defined above other than $R V_{t}$ (e.g., $R V J P_{t}, R V J N_{t}, S R V J_{t}$, etc.). Hereafter, we shall drop the superscript " $\mathrm{W}$ " for the sake of notational brevity. All the descriptors used to denote the various realized measures constructed in our empirical analysis are summarized in Table 1.

As described in detail in Section 4, the realized measures outlined above are used in several different ways in our empirical analysis. First, we carry out single portfolio sorts, in which we sort stock portfolios on the above realized jump measures, and predict average excess returns, one week ahead. In these experiments, we also calculate alphas based on regressions that use the Fama-French and Carhart factors. In this first part of our analysis, we also examine cumulative returns and Sharpe ratios. In addition to the single portfolio sorts, we carry out double portfolio sorts, in which we sort not only on realized jump risk measures, but also on various control variables, including realized skewness and other firm specific characteristics. Using these double-sorts, we also examine the inter-play between individual stock-level jump variations and industry-level jump variations. Needless to say, the purpose of our double-sorts is to examine the robustness of our findings based on single sorts, after controlling for other realized measures. Next, we carry out a series of Fama-MacBeth regressions, in order to check the robustness of our findings to the inclusion of various firm specific characteristics. Finally, we carry out an event study in which the effect of earning surprises on realized jump measures is examined. For complete details, see Section 4.

Table 1. Realized Measures and Firm Characteristics.

\begin{tabular}{ll}
\hline Panel A: & Realized Measures Used in Portfolio Sorts and Fama-MacBeth Regressions \\
\hline RVJP & Positive (upside) jump variation, see (1). \\
RVJN & Negative (downside) jump variation, see (2). \\
SRVJ & Signed jump variation, $R V J P-R V J N$, see (3). \\
RVLJP & Positive (upside) large jump variation, see (4). \\
RVLJN & Negative (downside) large jump variation, see (5). \\
SRVLJ & Signed large jump variation, $R V L J P-R V L J N$, see (6). \\
RVSJP & Positive (upside) small jump variation, see (7). \\
RVSJN & Negative (downside) small jump variation, see (8). \\
SRVSJ & Signed small jump variation, $R V S J P-R V S J N$, see (9). \\
RVOL & Realized volatility \\
RSK & Realized skewness, see (12). \\
RKT & Realized kurtosis, see (13). \\
\hline Panel B: & Explanatory Variables and Firm Characteristics Used in Fama-MacBeth Regressions \\
\hline BETA & Market beta \\
log(Size) & Natural logarithm of firm size \\
BEME & Book-to-market ratio \\
MOM & Momentum \\
REV & Short-term reversal \\
IVOL & Idiosyncratic volatility \\
CSK & Co-skewness \\
CKT & Co-kurtosis \\
MAX & Maximum daily return \\
MIN & Minimum daily return \\
ILLIQ & Illiquidity \\
\hline Notes: The realized measures listed in Panel A of this table are defined and discussed in Section 2. For detailed \\
descriptions \\
Bollerslev et al. (2020), and the references cited therein. \\
\end{tabular}

\section{Data}

We use high-frequency trading data obtained from the consolidated Trade and Quote (TAQ) database. In particular, we analyze all stocks in the TAQ database that are listed on the NYSE, Amex, and NASDAQ stock exchanges. There are 15,585 unique stocks during the 1246 weeks analyzed in this 
paper. ${ }^{11}$ The sample period is from 4 January 1993 to 31 December 2016. Intraday prices are sampled at 5-min intervals from 9:30 a.m. to 4:00 p.m. from Monday to Friday. Overnight returns are not considered in this paper, and days with less than 80 transactions at a 5-min frequency are eliminated. All high-frequency data used in this paper are cleaned to remove trades outside of exchange hours, negative or zero prices or volumes, trade corrections and non-standard sale conditions, using the methodology described in Appendix A.1 in Bollerslev et al. (2020).

We constructed two variants of our dataset. The first is cleaned as discussed above. The second classifies 5 -min intraday returns greater than $15 \%$ as abnormal and replaces them with zeros. In the sequel, results based on analysis of the second dataset are reported. However, results based on the use of the first dataset are qualitatively the same; and indeed key return results reported in this paper generally change by 1 basis point or less when the former dataset is used in our analysis. Complete results are available upon request from the authors.

Daily and monthly returns, and adjusted numbers of shares for individual securities are collected from the CRSP database. Delisting returns in CRSP are used as returns after the last trading day. Daily Fama-French and Carhart four-factor (FFC) portfolio returns are obtained from Kenneth R. French's website.

Following Amaya et al. (2015) and Bollerslev et al. (2020), we also construct various lower frequency firm-level variables that might be related to future returns, such as the market beta (BETA), the firm size, the book-to-market ratio (BEME), momentum (MOM), short-term reversals (REV), idiosyncratic volatility (IVOL), co-skewness (CSK), co-kurtosis (CKT), maximum (MAX) and minimum (MIN) daily return in the previous week, and the Amihud (2002) illiquidity measure (ILLIQ). For a complete list of these firm specific control variables, refer to Table 1. For a detailed description of these variables, including the methodology used to construct them, see Appendix A.2 in Bollerslev et al. (2020).

Please note that while the majority of our analysis is based on the examination of individual stocks, in our double-sorts, there are some cases (that are reported in Section 4.4) where we examine the inter-play between individual stock-level jump variations and industry-level jump variations, as mentioned above. In this case, we follow the Fama-French industry classification approach, and group stocks into 49 industries based on their SIC codes, which are obtained from CRSP.

\subsection{Unconditional Distributions of Realized Measures}

Figure 1 displays kernel density estimates of the unconditional distributions of each of our realized measures, across all firms and weeks. The top two panels in the figure show the distributions of signed jump variation and realized skewness. Both distributions are approximately symmetric and peaked around zero. The skewness distribution is more fat-tailed, however. ${ }^{12}$ The middle two panels of Figure 1 display the distributions of signed small and large jump variation. Similar to signed jump variation, both signed small and large jump variation measures are approximately symmetric around zero, but signed small jump variation is less fat-tailed. ${ }^{13}$ Consistent with the results in Amaya et al. (2015) and Bollerslev et al. (2020), realized volatility and realized kurtosis are both right skewed and very fat-tailed, as shown in the bottom two panels of the figure. ${ }^{14}$

Figure 2 shows the time variation in the cross-sectional distribution of each realized measure using 10-week moving averages. In particular, 10th, 50th, and 90th percentiles for each realized measure in the cross-section are plotted. Thus, dispersion at any given time in these plots reflects

11 In some cases, multiple TAQ symbols are matched with a unique Center for Research in Security Prices (CRSP) PERMNO. Over each quarter, the TAQ symbol which has the most observations is kept and the other overlapping observations are dropped.

12 The kurtosis of signed jump variation is 4.36. For realized skewness, the analogous statistic is 12.04 .

13 The unconditional kurtosis is 6.43 and 3.51 , for signed small and large jump variation, based on truncation level $\gamma^{1}$; and 8.87 and 3.09 based on truncation level $\gamma^{2}$, respectively.

14 The kurtosis is 15.85 and 27.24 , for the unconditional distribution of realized volatility and realized kurtosis, respectively. 
information about the cross-sectional distribution of the realized measure. Inspection of Panels A and $B$ in the figure reveals that signed jump variation and realized skewness have stable dispersion, for all three cross-sectional percentiles, over time, while the cross-sectional dispersion in realized volatility and kurtosis are rather time-dependent (see Panels C and D). Additionally, similar to the cross-sectional distribution of signed jump variation, the percentiles for signed small and large jump variation measures are quite steady over time, as indicated in Panels E- $\mathrm{H}$.

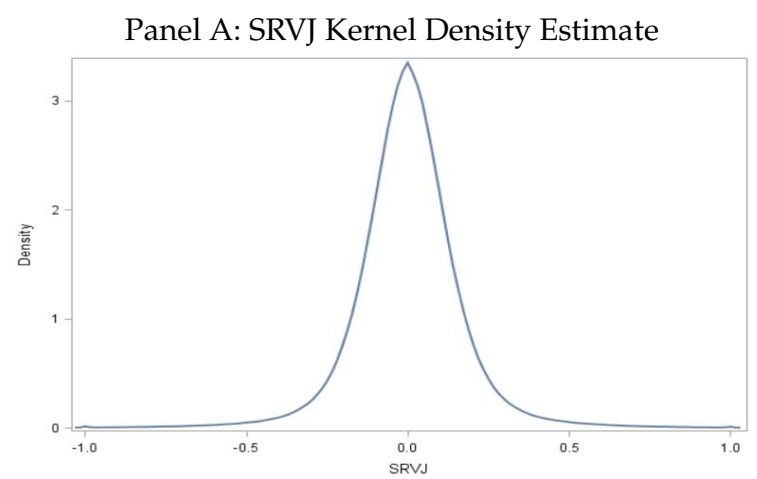

Panel C: SRVLJ Kernel Density Estimate

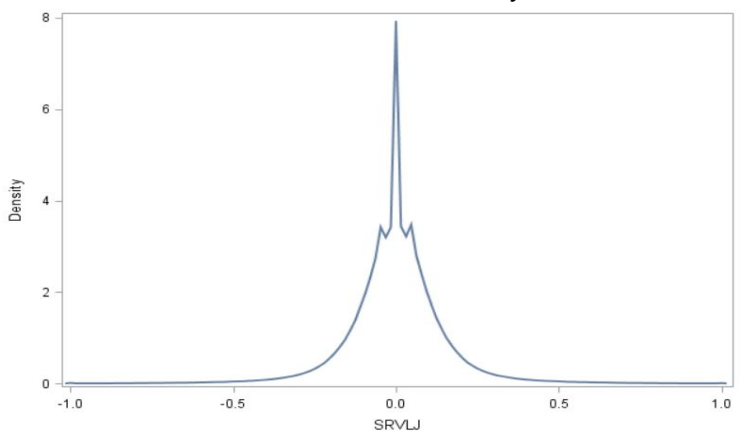

Panel E: RKT Kernel Density Estimate

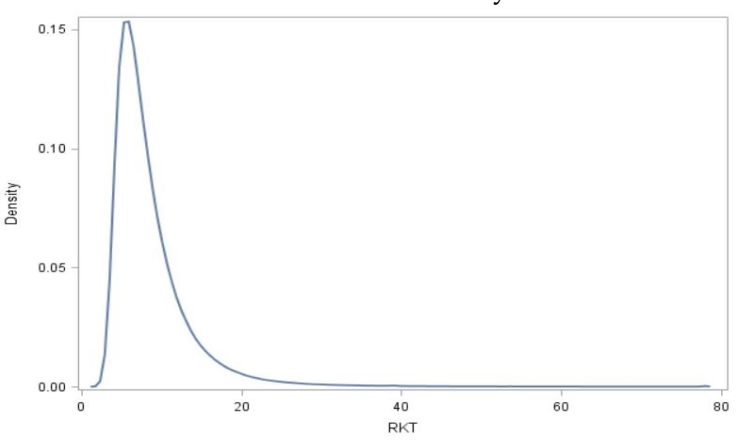

Panel B: RSK Kernel Density Estimate

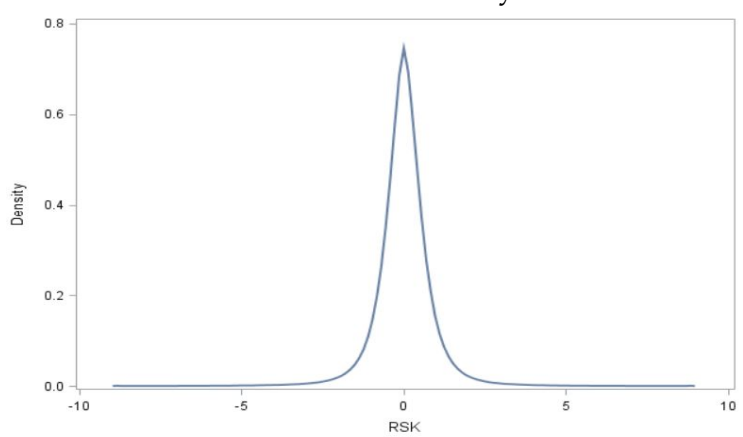

Panel D: SRVSJ Kernel Density Estimate

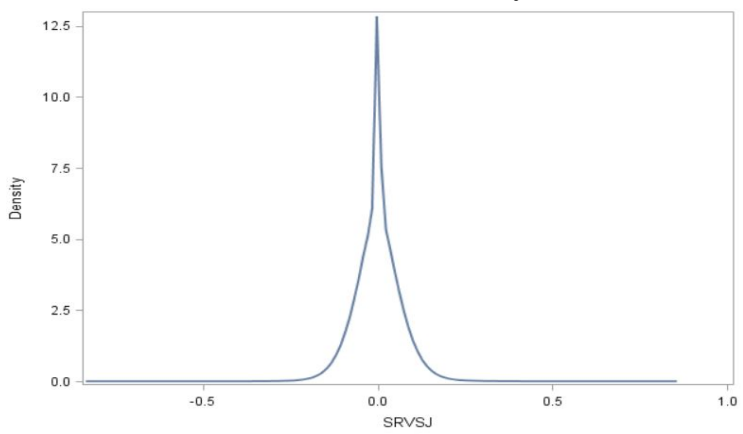

Panel F: RVOL Kernel Density Estimate

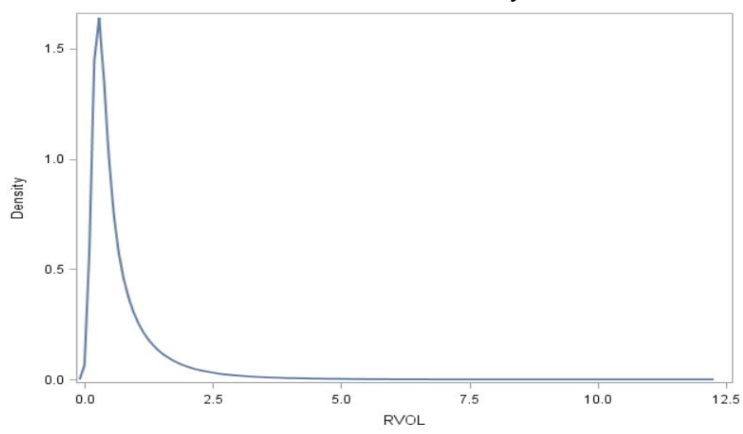

Figure 1. Unconditional Distributions of Realized Measures. Panels A-F display unconditional distribution kernel density estimates of various realized measures, for the cross-section of stock returns for the period January 1993 to December 2016. Signed small and large jump variation measures are constructed using truncation levels $\gamma^{1}=4 \sqrt{\frac{1}{t} \widehat{I V}_{t}^{(i)}} \Delta_{n}^{0.49}$. Distributions are similar when using $\gamma^{2}=5 \sqrt{\frac{1}{t} \widehat{I V}_{t}^{(i)}} \Delta_{n}^{0.49}$ 
Panel A: Percentiles of SRVJ

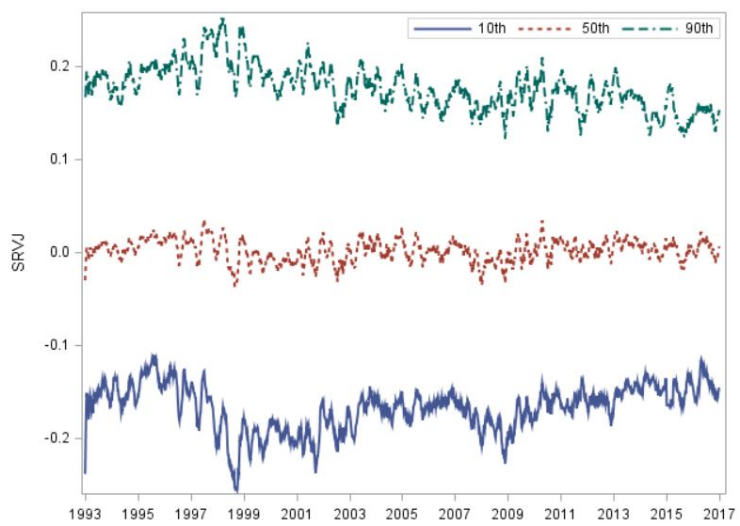

Panel C: Percentiles of RKT

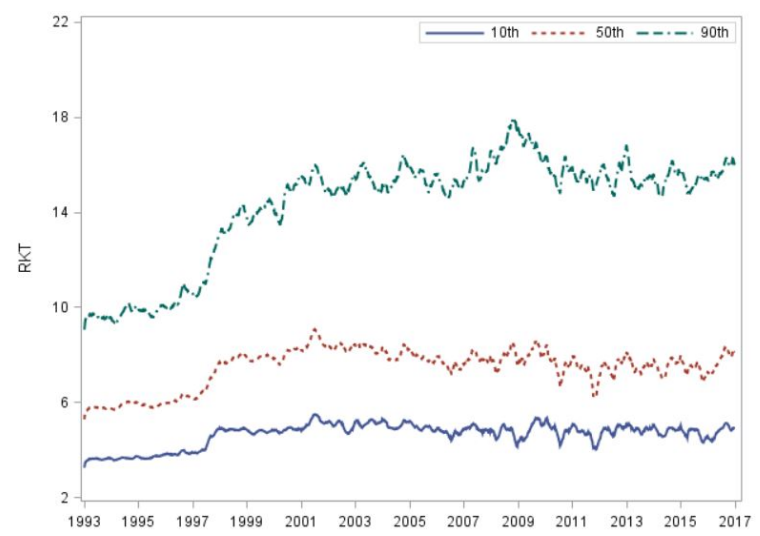

Panel E: Percentiles of SRVLJ Based on $\gamma^{2}$

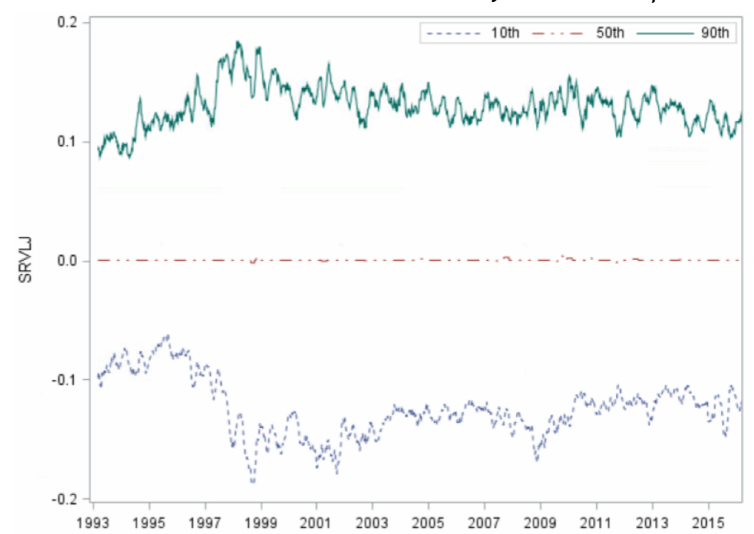

Panel B: Percentiles of RSK

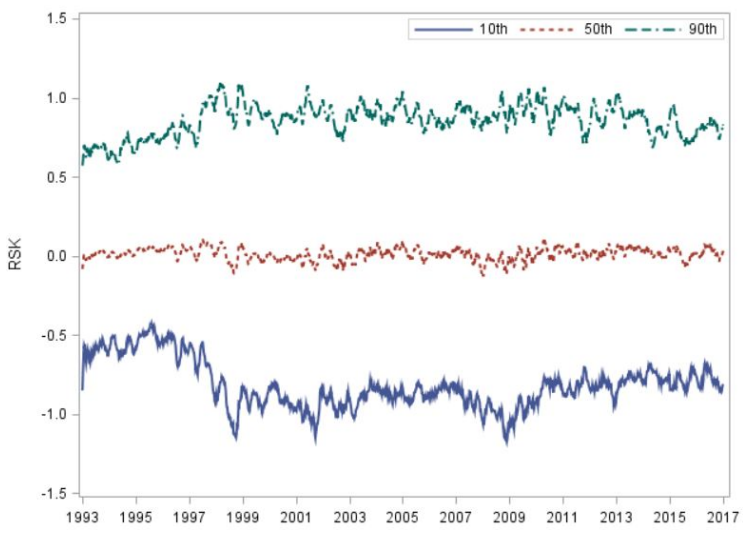

Panel D: Percentiles of RVOL

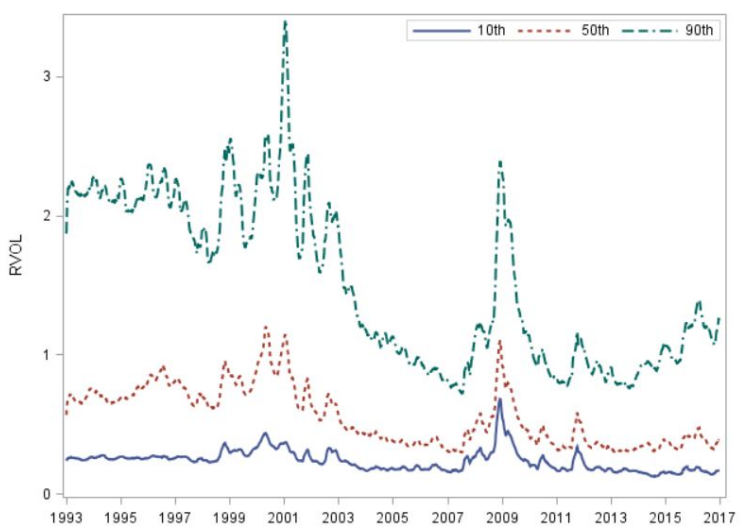

Panel F: Percentiles of SRVSJ Based on $\gamma^{2}$

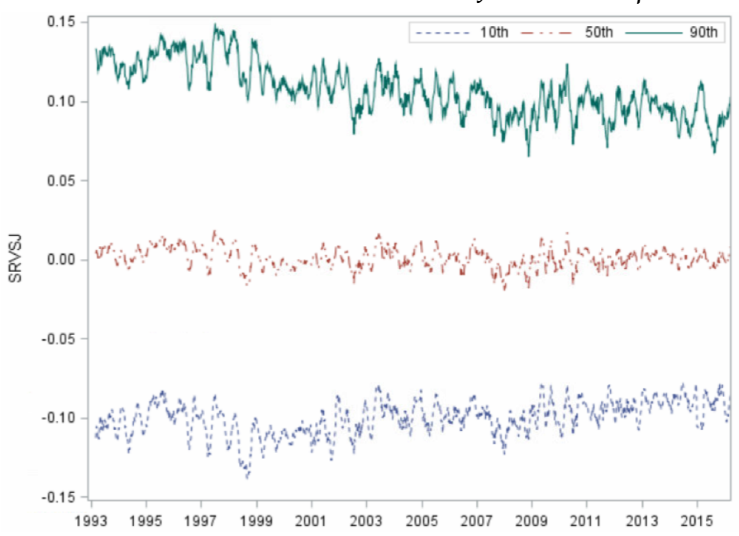

Figure 2. Percentiles of Realized Measures. Panels A-H display 10-week moving averages of percentiles of realized measures, for the cross-section of stocks, for the period January 1993 to December 2016. Signed small and large jump variation measures are contructed based on jump truncation level $\gamma^{2}=5 \sqrt{\frac{1}{t} \widehat{I V}_{t}^{(i)}} \Delta_{n}^{0.49}$.

\subsection{Summary Statistics and Portfolio Characteristics}

Table 2 contains various summary statistics for all the realized measures summarized in Table 1. In Panel A, the cross-sectional means and standard errors for each of the realized measures is given. This is done for two different truncation levels, denoted as $\gamma^{1}=4 \sqrt{\frac{1}{t} \widehat{I V}_{t}^{(i)}} \Delta_{n}^{0.49}$ and $\gamma^{2}=5 \sqrt{\frac{1}{t} \widehat{I V}_{t}^{(i)}} \Delta_{n}^{0.49}$. As might be expected, jump variation is quite sensitive to the choice of $\gamma$. 
For example, the (normalized) mean of RVSJP (positive (upside) small jump variation) increases from 0.1180 to 0.1715 when the threshold is increased from $\gamma^{1}$ to $\gamma^{2}$.

Panel B of Table 2 contains cross-sectional correlations for all the realized measures. In accord with the findings reported by Amaya et al. (2015) and Bollerslev et al. (2020), signed jump variation (SRVJ) and realized skewness (RSK) are highly correlated with each other and have significantly positive correlations with the short-term reversal variable (REV); as well as with maximum (MAX) and minimum (MIN) daily returns in the previous week.

Interestingly, we also find that signed large jump variation (SRVLJ) is highly correlated with SRVJ and with RSK. However, signed small jump variation (SRVSJ) has a lower correlation with SRVJ and much smaller positive correlation with RSK. This finding is consistent with our finding discussed below that realized skewness captures information that is primarily contained in large jumps; and serves as an important distinction between the findings in this paper and those reported in the papers discussed above.

Table 3 complements Table 2 by sorting stocks into quintile portfolios based on different realized measures. On each Tuesday, stocks are ranked by the realized variation measures, and we calculate the equal-weighted averages of each firm characteristic in the same week. Panels A, B, C and E report summary statistics for portfolios sorted by SRVJ, SRVLJ, SRVSJ, and RSK, respectively. Consistent with the correlations contained in Table 2, firms with larger signed small and large jump variation measures tend to have higher signed jump variation, realized skewness, REV, MAX and MIN. Firms with high realized volatility and realized kurtosis (see Panels D and F) tend to be illiquid and small. ${ }^{15}$

15 See the Supplementary Appendix for results based on the examination of additional quintile portfolios that are constructed based on ex-ante risk measures and displayed with ex-post risk measures. It is clear that sorting stocks based on jump risk measures results in portfolios with the desired risk exposures. 
Table 2. Summary Statistics for Various Realized Measures and Firm Characteristics Based on Two Jump Truncation Levels.

\begin{tabular}{|c|c|c|c|c|c|c|c|c|c|c|c|c|c|c|c|c|c|c|c|c|c|c|c|}
\hline \multicolumn{24}{|c|}{ Panel A: Cross-Sectional Summary Statistics } \\
\hline & SRVJ & RVJP & RVJN & SRVLJ & RVLJP & RVLJN & SRVSJ & RVSJP & RVSJN & RVOL & RSK & RKT & BETA & $\log ($ Size $)$ & BEME & MOM & REV & IVOL & CSK & CKT & MAX & MIN & ILLIQ \\
\hline \multicolumn{24}{|c|}{ Part I: Jump Truncation Level $=\gamma^{1}$} \\
\hline Mean & 0.0061 & 0.2698 & 0.2637 & 0.0045 & 0.1518 & 0.1472 & 0.0015 & 0.1180 & 0.1165 & 0.9489 & 0.0288 & 8.2569 & 1.0794 & 6.5280 & 0.5969 & 2023.8456 & 70.6077 & 0.0293 & -0.0263 & 1.1438 & 412.1094 & -346.7608 & -5.2826 \\
\hline Std & 0.1537 & 0.1350 & 0.1347 & 0.1424 & 0.1555 & 0.1523 & 0.0635 & 0.0783 & 0.0783 & 2.1211 & 0.8159 & 4.5706 & 0.5566 & 1.8359 & 0.7224 & 7464.5273 & 927.3551 & 0.0250 & 0.3283 & 0.8474 & 572.1454 & 359.6789 & 2.4047 \\
\hline \multicolumn{24}{|c|}{ Part II: Jump Truncation Level $=\gamma^{2}$} \\
\hline Mean & 0.0061 & 0.2698 & 0.2637 & 0.0029 & 0.0983 & 0.0954 & 0.0031 & 0.1715 & 0.1684 & 0.9489 & 0.0288 & 8.2569 & 1.0794 & 6.5280 & 0.5969 & 2023.8456 & 70.6077 & 0.0293 & -0.0263 & 1.1438 & 412.1094 & -346.7608 & -5.2826 \\
\hline Std & 0.1537 & 0.1350 & 0.1347 & 0.1303 & 0.1401 & 0.1368 & 0.0859 & 0.0911 & 0.0909 & 2.1211 & 0.8159 & 4.5706 & 0.5566 & 1.8359 & 0.7224 & 7464.5273 & 927.3551 & 0.0250 & 0.3283 & 0.8474 & 572.1454 & 359.6789 & 2.4047 \\
\hline \multicolumn{24}{|c|}{ Panel B: Cross-Sectional Correlations } \\
\hline & SRVJ & RVJP & RVJN & SRVLJ & RVLJP & RVLJN & SRVSJ & RVSJP & RVSJN & RVOL & RSK & RKT & BETA & $\log ($ Size $)$ & BEME & MOM & REV & IVOL & CSK & CKT & MAX & MIN & ILLIQ \\
\hline \multicolumn{24}{|c|}{ Part I: Jump Truncation Level $=\gamma^{1}$} \\
\hline SRVJ & 1.00 & 0.57 & -0.57 & 0.91 & 0.43 & -0.40 & 0.37 & 0.13 & -0.18 & -0.02 & 0.94 & 0.03 & -0.03 & 0.01 & 0.01 & 0.01 & 0.30 & -0.03 & 0.09 & 0.00 & 0.17 & 0.22 & 0.00 \\
\hline RVJP & & 1.00 & 0.33 & 0.52 & 0.85 & 0.37 & 0.21 & 0.04 & -0.13 & 0.22 & 0.54 & 0.45 & -0.26 & -0.49 & 0.14 & -0.10 & 0.15 & 0.12 & 0.04 & -0.24 & 0.15 & 0.06 & 0.56 \\
\hline RVJN & & & 1.00 & -0.52 & 0.35 & 0.84 & -0.22 & -0.10 & 0.08 & 0.24 & -0.54 & 0.41 & -0.23 & -0.49 & 0.13 & -0.11 & -0.19 & 0.15 & -0.06 & $\begin{array}{l}-0.24 \\
-0.24\end{array}$ & -0.05 & -0.20 & 0.55 \\
\hline SRVLJ & & & & 1.00 & 0.48 & -0.44 & -0.04 & -0.05 & -0.01 & -0.01 & 0.92 & 0.03 & -0.02 & 0.00 & 0.01 & 0.00 & 0.20 & -0.02 & 0.05 & 0.00 & 0.12 & 0.16 & 0.00 \\
\hline RVLJP & & & & & 1.00 & 0.57 & -0.02 & -0.46 & -0.45 & 0.23 & 0.44 & 0.61 & -0.25 & -0.47 & 0.12 & -0.06 & 0.09 & 0.13 & 0.02 & -0.24 & 0.12 & -0.02 & 0.54 \\
\hline RVLJN & & & & & & 1.00 & 0.01 & -0.44 & -0.45 & 0.24 & -0.41 & 0.59 & -0.23 & -0.48 & 0.11 & -0.06 & -0.10 & 0.15 & -0.03 & -0.24 & 0.01 & -0.17 & 0.54 \\
\hline SRVSJ & & & & & & & 1.00 & 0.42 & -0.42 & -0.02 & 0.19 & 0.00 & -0.03 & 0.01 & 0.01 & 0.01 & 0.26 & -0.03 & 0.08 & 0.00 & 0.14 & 0.18 & 0.00 \\
\hline $\begin{array}{l}\text { RVSJP } \\
\text { RVD }\end{array}$ & & & & & & & 1.00 & $\begin{array}{l}0.42 \\
1.00\end{array}$ & $\begin{array}{c}-0.42 \\
0.64\end{array}$ & $\begin{array}{l}-0.02 \\
-0.04\end{array}$ & 0.06 & $\begin{array}{l}0.00 \\
-0.40\end{array}$ & $\begin{array}{l}-0.06 \\
0.06\end{array}$ & $\begin{array}{l}0.01 \\
0.03\end{array}$ & $\begin{array}{l}0.01 \\
0.01\end{array}$ & $\begin{array}{l}0.01 \\
-0.05\end{array}$ & $\begin{array}{l}0.20 \\
0.10\end{array}$ & $\begin{array}{l}-0.03 \\
-0.03\end{array}$ & $\begin{array}{l}0.00 \\
0.04\end{array}$ & 0.04 & $\begin{array}{l}0.14 \\
0.02\end{array}$ & 0.11 & $\begin{array}{l}0.06 \\
-0.06\end{array}$ \\
\hline RVSJN & & & & & & & & & 1.00 & -0.03 & -0.10 & -0.40 & 0.08 & 0.02 & 0.01 & -0.06 & -0.12 & -0.01 & -0.03 & 0.03 & -0.09 & -0.04 & -0.06 \\
\hline RVOL & & & & & & & & & & 1.00 & -0.01 & 0.22 & -0.05 & -0.55 & 0.08 & -0.12 & 0.06 & 0.56 & -0.01 & -0.27 & 0.44 & -0.47 & 0.56 \\
\hline RSK & & & & & & & & & & & 1.00 & 0.04 & -0.02 & 0.00 & 0.01 & 0.00 & 0.22 & -0.02 & 0.06 & 0.00 & 0.13 & 0.17 & 0.00 \\
\hline RKT & & & & & & & & & & & & 1.00 & -0.20 & -0.34 & 0.09 & -0.02 & 0.00 & 0.10 & -0.01 & -0.19 & 0.08 & -0.10 & 0.40 \\
\hline BETA & & & & & & & & & & & & & 1.00 & 0.10 & -0.09 & 0.00 & -0.04 & 0.06 & 0.01 & 0.30 & 0.03 & -0.09 & -0.16 \\
\hline ME & & & & & & & & & & & & & & 1.00 & -0.19 & 0.11 & -0.05 & -0.52 & 0.01 & 0.40 & -0.32 & 0.35 & -0.93 \\
\hline BEME & & & & & & & & & & & & & & & 1.00 & 0.03 & 0.02 & 0.05 & 0.00 & $\begin{array}{l}-0.06 \\
-0.06\end{array}$ & 0.05 & -0.03 & 0.18 \\
\hline MOM & & & & & & & & & & & & & & & & 1.00 & 0.00 & -0.08 & -0.07 & 0.06 & -0.05 & 0.05 & -0.15 \\
\hline REV & & & & & & & & & & & & & & & & & 1.00 & 0.12 & 0.16 & -0.04 & 0.49 & 0.29 & 0.05 \\
\hline IVOL & & & & & & & & & & & & & & & & & & 1.00 & 0.02 & -0.35 & 0.50 & -0.47 & 0.47 \\
\hline CSK & & & & & & & & & & & & & & & & & & & 1.00 & 0.01 & 0.07 & 0.07 & 0.00 \\
\hline CKT & & & & & & & & & & & & & & & & & & & & 1.00 & -0.16 & 0.15 & -0.37 \\
\hline MAX & & & & & & & & & & & & & & & & & & & & & 1.00 & -0.28 & 0.34 \\
\hline MIN & & & & & & & & & & & & & & & & & & & & & & 1.00 & -0.35 \\
\hline ILLIQ & & & & & & & & & & & & & & & & & & & & & & & 1.00 \\
\hline & SRVJ & RVJP & RVJN & SRVLJ & RVLJP & RVLJN & SRVSJ & RVSJP & RVSJN & RVOL & RSK & RKT & BETA & $\log ($ Size) & BEME & MOM & REV & IVOL & CSK & CKT & MAX & MIN & ILLIQ \\
\hline \multicolumn{24}{|c|}{ Part II: Jump Truncation Level $=\gamma^{2}$} \\
\hline SRVJ & 1.00 & 0.57 & -0.57 & 0.83 & 0.40 & -0.37 & 0.52 & 0.23 & -0.27 & -0.02 & 0.94 & 0.03 & -0.03 & 0.01 & 0.01 & 0.01 & 0.30 & -0.03 & 0.09 & 0.00 & 0.17 & 0.22 & 0.00 \\
\hline RVJP & & 1.00 & 0.33 & 0.48 & 0.77 & 0.33 & 0.30 & 0.30 & 0.01 & 0.22 & 0.54 & 0.45 & -0.26 & -0.49 & 0.14 & -0.10 & 0.15 & 0.12 & 0.04 & -0.24 & 0.15 & 0.06 & 0.56 \\
\hline RVJN & & & 1.00 & -0.47 & 0.31 & 0.75 & -0.31 & 0.03 & 0.33 & 0.24 & -0.54 & 0.41 & -0.23 & -0.49 & 0.13 & -0.11 & -0.19 & 0.15 & -0.06 & -0.24 & -0.05 & -0.20 & 0.55 \\
\hline SRVLJ & & & & $\begin{array}{c}-0.4 \% \\
1.00\end{array}$ & $\begin{array}{l}.31 \\
0.49\end{array}$ & -0.44 & $\begin{array}{l}-0.01 \\
-0.04\end{array}$ & -0.05 & -0.01 & $\begin{array}{l}0.24 \\
-0.01\end{array}$ & $\begin{array}{c}-0.044 \\
0.89\end{array}$ & $\begin{array}{l}0.41 \\
0.04\end{array}$ & $\begin{array}{l}-0.20 \\
-0.01\end{array}$ & $\begin{array}{c}-0.49 \\
0.00\end{array}$ & 0.01 & 0.00 & 0.16 & -0.02 & 0.04 & $\begin{array}{c}-0.24 \\
0.00\end{array}$ & $\begin{array}{c}-0.09 \\
0.09\end{array}$ & 0.13 & 0.00 \\
\hline RVLJP & & & & & 1.00 & 0.56 & -0.02 & -0.36 & -0.34 & 0.20 & 0.43 & 0.64 & -0.24 & -0.40 & 0.11 & -0.05 & 0.06 & 0.11 & 0.01 & -0.22 & 0.10 & -0.02 & 0.47 \\
\hline $\begin{array}{l}\text { RVLJN } \\
\text { S }\end{array}$ & & & & & & 1.00 & 0.01 & -0.34 & -0.35 & 0.21 & -0.40 & 0.6 & -0.23 & -0.41 & 0. & -0 & -0.09 & 0.7 & -0.03 & -0 & 0.01 & -0.14 & 0.47 \\
\hline $\begin{array}{l}\text { SRVSJ } \\
\text { S }\end{array}$ & & & & & & & $\begin{array}{l}0.01 \\
1.00\end{array}$ & 0.47 & -0.47 & $\begin{array}{l}0.21 \\
-0.02\end{array}$ & 0.32 & 0.8 & -0.03 & 0. & 0.0 & 0. & 0.30 & -0.03 & 0.09 & 0.00 & 0.16 & 0.21 & 0.00 \\
\hline RVSJP & & & & & & & & 1.00 & 0.55 & 0.03 & 0.13 & -0.30 & -0.02 & -0.14 & 0.04 & -0.07 & 0.13 & 0.02 & 0.04 & -0.04 & 0.07 & 0.10 & 0.13 \\
\hline RVSJN & & & & & & & & & 1.00 & 0.05 & -0.17 & -0.30 & 0.01 & -0.15 & 0.04 & -0.07 & -0.15 & 0.05 & -0.04 & -0.04 & -0.08 & -0.09 & 0.13 \\
\hline RVOL & & & & & & & & & & 1.00 & -0.01 & 0.22 & -0.05 & -0.55 & 0.08 & -0.12 & 0.06 & 0.56 & -0.01 & -0.27 & 0.44 & -0.47 & 0.56 \\
\hline $\begin{array}{l}\text { RSK } \\
\text { RS }\end{array}$ & & & & & & & & & & & 1.00 & 0.04 & -0.02 & 0.00 & 0.01 & 0.00 & 0.22 & -0.02 & 0.06 & 0.00 & 0.13 & 0.17 & 0.00 \\
\hline $\begin{array}{l}\text { RKT } \\
\text { R }\end{array}$ & & & & & & & & & & & & 1.00 & -0.20 & -0.34 & 0.09 & -0.02 & 0.00 & 0.10 & -0.01 & -0.19 & 0.08 & -0.10 & 0.40 \\
\hline
\end{tabular}


Table 2. Cont.

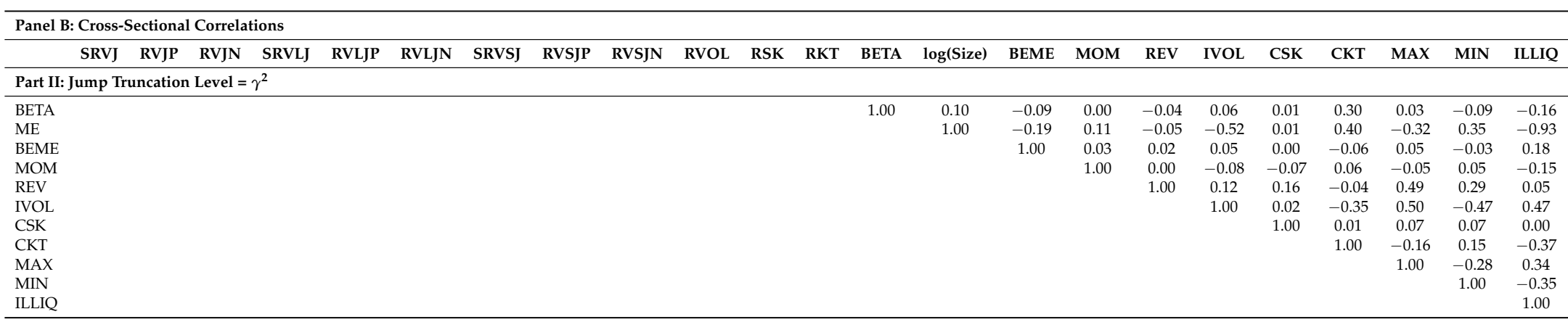

Notes: See notes to Table 1. This table presents cross-sectional summary statistics and correlations for all realized measures and control variables based on two truncation levels $\gamma^{1}=4 \sqrt{\frac{1}{t} \widehat{I V}_{t}^{(i)}} \Delta_{n}^{0.49}$ and $\gamma^{2}=5 \sqrt{\frac{1}{t} \widehat{I V}_{t}^{(i)}} \Delta_{n}^{0.49}$. The entries in the table for realized measures (see columns 2-13) are constructed using 5-min intraday high-frequency data. Entries for firm characteristics (see columns 14-24) are constructed using daily data, with the exception of BEME, which is constructed using monthly data. For complete details, see Sections 3 and 4 .

Table 3. Realized Measures and Firm Characteristics of Portfolios Sorted by Various Realized Measures.

\begin{tabular}{|c|c|c|c|c|c|c|c|c|c|c|c|c|c|c|c|c|c|c|c|c|c|c|c|}
\hline \multicolumn{24}{|c|}{ Panel A: Stocks Sorted by SRVJ } \\
\hline Quintile & RVJP & RVJN & RVLJP & RVLJN & RVSJP & RVSJN & SRVLJ & SRVSJ & SRVJ & RVOL & RSK & RKT & BETA & $\log ($ Size) & BEME & MOM & REV & IVOL & CSK & CKT & MAX & MIN & ILLIQ \\
\hline 1 & 0.2021 & 0.3959 & 0.1161 & 0.2723 & 0.0860 & 0.1235 & -0.1563 & -0.0375 & -0.1938 & 0.9394 & -0.9324 & 9.8720 & 1.0369 & 6.1326 & 0.6235 & 0.2006 & -0.0363 & 0.0317 & -0.0708 & 1.0482 & 0.0295 & -0.0494 & -4.6903 \\
\hline 2 & 0.2200 & 0.2777 & 0.1015 & 0.1391 & 0.1185 & 0.1385 & -0.0376 & -0.0200 & -0.0576 & 0.9513 & -0.2504 & 7.1729 & 1.1351 & 6.7041 & 0.5711 & 0.2051 & -0.0111 & 0.0293 & -0.0443 & 1.1987 & 0.0346 & -0.0383 & -5.5835 \\
\hline 3 & 0.2435 & 0.2399 & 0.1127 & 0.1103 & 0.1308 & 0.1296 & 0.0023 & 0.0012 & 0.0036 & 1.0360 & 0.0194 & 6.9162 & 1.1301 & 6.8171 & 0.5695 & 0.2030 & 0.0077 & 0.0283 & -0.0242 & 1.2222 & 0.0397 & -0.0328 & -5.7301 \\
\hline 4 & 0.2801 & 0.2138 & 0.1441 & 0.1005 & 0.1360 & 0.1132 & 0.0436 & 0.0227 & 0.0663 & 0.9162 & 0.2954 & 7.2497 & 1.1096 & 6.7855 & 0.5778 & 0.2097 & 0.0266 & 0.0278 & -0.0066 & 1.2055 & 0.0454 & -0.0282 & -5.6668 \\
\hline 5 & 0.4035 & 0.1914 & 0.2846 & 0.1138 & 0.1189 & 0.0775 & 0.1708 & 0.0413 & 0.2121 & 0.9018 & 1.0138 & 10.0739 & 0.9851 & 6.2007 & 0.6427 & 0.1936 & 0.0485 & 0.0293 & 0.0145 & 1.0443 & 0.0569 & -0.0247 & -4.7419 \\
\hline \multicolumn{24}{|c|}{ Panel B: Stocks Sorted by SRVLJ } \\
\hline Quintile & RVJP & RVJN & $\begin{array}{l}\text { RVLJP } \\
\end{array}$ & RVLJN & RVSJP & RVSJN & SRVLJ & SRVSJ & SRVJ & RVOL & RSK & RKT & BETA & $\log ($ Size $)$ & BEME & MOM & REV & IVOL & CSK & CKT & MAX & MIN & $\begin{array}{l}\text { ILLIQ } \\
\end{array}$ \\
\hline \multicolumn{24}{|c|}{ Part I: Jump Truncation Level $=\gamma^{1}$} \\
\hline 1 & 0.2255 & 0.3959 & 0.1250 & 0.3016 & 0.1005 & 0.0943 & -0.1766 & 0.0062 & -0.1703 & 0.9429 & -0.9075 & 10.2967 & 1.0163 & 6.0403 & 0.6329 & 0.1966 & -0.0221 & 0.0316 & -0.0548 & 1.0357 & 0.0347 & -0.0461 & -4.5515 \\
\hline 2 & 0.2222 & 0.2603 & 0.0874 & 0.1297 & 0.1347 & 0.1307 & -0.0422 & 0.0041 & -0.0382 & 0.9128 & -0.2003 & 6.9378 & 1.1314 & 6.7639 & 0.5678 & 0.2104 & -0.0011 & 0.0288 & -0.0329 & 1.2050 & 0.0374 & -0.0357 & -5.6652 \\
\hline 3 & 0.2296 & 0.2252 & 0.0968 & 0.0936 & 0.1329 & 0.1316 & 0.0032 & 0.0013 & 0.0044 & 1.1510 & 0.0218 & 6.5571 & 1.1308 & 6.9059 & 0.5648 & 0.1997 & 0.0069 & 0.0282 & -0.0245 & 1.2380 & 0.0390 & -0.0326 & -5.8581 \\
\hline 4 & 0.2726 & 0.2229 & 0.1493 & 0.0981 & 0.1233 & 0.1248 & 0.0512 & -0.0016 & 0.0497 & 0.8314 & 0.2602 & 7.2170 & 1.1237 & 6.7993 & 0.5764 & 0.2118 & 0.0169 & 0.0280 & -0.0166 & 1.2086 & 0.0421 & -0.0304 & -5.6962 \\
\hline 5 & 0.4041 & 0.2153 & 0.3149 & 0.1234 & 0.0891 & 0.0920 & 0.1915 & -0.0028 & 0.1887 & 0.9158 & 0.9900 & 10.5179 & 0.9873 & 6.0946 & 0.6471 & 0.1887 & 0.0354 & 0.0299 & -0.0021 & 1.0376 & 0.0533 & -0.0284 & -4.6003 \\
\hline \multicolumn{24}{|c|}{ Part II: Jump Truncation Level $=\gamma^{2}$} \\
\hline 1 & 0.2412 & 0.3897 & 0.0841 & 0.2403 & 0.1571 & 0.1494 & -0.1561 & 0.0076 & -0.1485 & 0.9355 & -0.8465 & 10.5599 & 1.0128 & 6.0603 & 0.6360 & 0.1928 & -0.0152 & 0.0313 & -0.0478 & 1.0403 & 0.0366 & -0.0438 & -4.5663 \\
\hline 2 & 0.2189 & 0.2314 & & & & 0.1794 & -0.0178 & 0.0054 & -0.0124 & 0.9321 & -0.0762 & 6.4172 & 1.1366 & 6.8798 & 0.5622 & 0.2132 & 0.0045 & 0.0283 & -0.0278 & 1.2310 & 0.0388 & -0.0340 & -5.8322 \\
\hline 3 & 0.243 & 0.240 & 0.080 & 0.07 & 0.16 & 0.16 & $0.0 c$ & -0.0 & 0.003 & 1.68 & 0.02 & 7.74 & 1.09 & 6.58 & 0.62 & 0.16 & 0.0063 & 0.03 & -0.0021 & 1.2281 & 0.0426 & -0.0360 & -5.3661 \\
\hline 4 & 0.2985 & 0.2611 & 0.1233 & 0.0854 & 0.1752 & 0.1756 & 0.0379 & -0.0004 & 0.0375 & 1.0724 & 0.2140 & 8.0742 & 1.0912 & 6.4686 & 0.5995 & 0.1944 & 0.0125 & 0.0294 & -0.0213 & 1.1478 & 0.0418 & -0.0324 & -5.2346 \\
\hline 5 & 0.3985 & 0.2321 & 0.2526 & 0.0837 & 0.1459 & 0.1484 & 0.1690 & -0.0025 & 0.1664 & 0.9201 & 0.9332 & 10.8242 & 0.9935 & 6.1033 & 0.6466 & 0.1896 & 0.0285 & 0.0300 & -0.0084 & 1.0418 & 0.0506 & -0.0301 & -4.6103 \\
\hline
\end{tabular}


Table 3. Cont

\begin{tabular}{|c|c|c|c|c|c|c|c|c|c|c|c|c|c|c|c|c|c|c|c|c|c|c|c|}
\hline \multicolumn{24}{|c|}{ Panel C: Stocks Sorted by SRVSJ } \\
\hline Quintile & RVJP & RVJN & RVLJP & RVLJN & RVSJP & RVSJN & SRVLJ & SRVSJ & SRVJ & RVOL & RSK & RKT & BETA & $\log ($ Size) & BEME & MOM & REV & IVOL & CSK & CKT & MAX & MIN & ILLIQ \\
\hline \multicolumn{24}{|c|}{ Part I: Jump Truncation Level $=\gamma^{1}$} \\
\hline 1 & 0.2060 & 0.2784 & 0.1085 & 0.0968 & 0.0975 & 0.1815 & 0.0116 & -0.0840 & -0.0724 & 0.7622 & -0.1832 & 7.1817 & 1.1514 & 6.7378 & 0.5654 & 0.2183 & -0.0270 & 0.0292 & -0.0637 & 1.2017 & 0.0302 & -0.0438 & -5.6348 \\
\hline 2 & 0.2711 & 0.2845 & 0.1758 & 0.1649 & 0.0952 & 0.1197 & 0.0110 & -0.0244 & -0.0135 & 1.0260 & -0.0157 & 8.6959 & 1.0715 & 6.4139 & 0.6062 & 0.1933 & -0.0046 & 0.0302 & -0.0417 & 1.1242 & 0.0380 & -0.0375 & -5.1168 \\
\hline 3 & 0.3077 & 0.3003 & 0.2186 & 0.2126 & 0.0891 & 0.0877 & 0.0060 & 0.0014 & 0.0074 & 1.2309 & 0.0345 & 9.7978 & 1.0053 & 6.1262 & 0.6382 & 0.1754 & 0.0066 & 0.0309 & -0.0275 & 1.0469 & 0.0423 & & -4.6619 \\
\hline 4 & 0.2743 & 0.2492 & 0.1413 & 0.1438 & 0.1330 & 0.1053 & -0.0026 & 0.0277 & 0.0251 & 0.9562 & 0.0709 & 8.0830 & 1.0906 & 6.6614 & 0.5854 & 0.2098 & 0.0190 & 0.0283 & -0.0099 & 1.1752 & 0.0436 & -0.0305 & -5.4837 \\
\hline 5 & 0.2830 & 0.1981 & 0.1004 & 0.1051 & 0.1826 & 0.0930 & -0.0047 & 0.0896 & 0.0849 & 0.7380 & 0.2395 & 7.1974 & 1.0947 & 6.8064 & 0.5783 & 0.2217 & 0.0428 & 0.0272 & 0.0136 & 1.1959 & 0.0517 & -0.0251 & -5.6801 \\
\hline \multicolumn{24}{|c|}{ Part II: Jump Truncation Level $=\gamma^{2}$} \\
\hline 1 & 0.2058 & 0.3081 & 0.0749 & 0.0650 & 0.1309 & 0.2431 & 0.0099 & -0.1121 & -0.1023 & 0.8104 & -0.3171 & 7.6436 & 1.1207 & 6.5435 & 0.5792 & 0.2179 & -0.0318 & 0.0301 & -0.0682 & 1.1558 & 0.0296 & -0.0461 & -5.3493 \\
\hline 2 & 0.2561 & 0.2841 & 0.1103 & 0.1027 & 0.1458 & 0.1814 & 0.0077 & -0.0356 & -0.0280 & 1.0119 & -0.0747 & 8.4222 & 1.0918 & 6.5103 & 0.5957 & 0.1964 & -0.0079 & 0.0298 & -0.0427 & 1.1482 & 0.0363 & -0.0379 & -5.2661 \\
\hline 3 & 0.2906 & 0.2847 & 0.1423 & 0.1381 & 0.1484 & 0.1465 & 0.0041 & 0.0019 & 0.0060 & 1.1729 & 0.0308 & 9.3041 & 1.0385 & 6.3412 & 0.6204 & 0.1788 & 0.0065 & 0.0301 & -0.0273 & 1.0955 & 0.0413 & -0.0346 & -4.9812 \\
\hline 4 & 0.2821 & 0.2444 & 0.0950 & 0.0979 & 0.1871 & 0.1465 & -0.0029 & 0.0405 & 0.0377 & 0.9731 & 0.1235 & 8.1884 & 1.0851 & 6.6358 & 0.5919 & 0.2046 & & 0.0284 & -0.0086 & 1.1700 & 0.0447 & & -5.4350 \\
\hline 5 & 0.3137 & 0.1959 & 0.0671 & 0.0713 & 0.2466 & 0.1246 & -0.0043 & 0.1220 & 0.1178 & 0.7734 & 0.3845 & $\begin{array}{l}7.6692 \\
7.669\end{array}$ & 1.0627 & 6.6238 & 0.5954 & 0.2153 & 0.0469 & 0.0278 & 0.0158 & 1.1527 & 0.0543 & -0.0245 & -5.4040 \\
\hline \multicolumn{24}{|c|}{ Panel D: Stocks Sorted by RVOL } \\
\hline Quintile & RVJP & RVJN & RVLJP & RVLJN & RVSJP & RVSJN & SRVLJ & SRVSJ & SRVJ & RVOL & RSK & RKT & BETA & $\log ($ Size) & BEME & MOM & REV & IVOL & CSK & CKT & MAX & MIN & ILLIQ \\
\hline 1 & 0.2255 & 0.2140 & 0.1013 & 0.0943 & 0.1241 & 0.1197 & 0.0070 & 0.0044 & 0.0114 & 0.2290 & 0.0485 & 6.8794 & 0.8390 & 8.3393 & 0.5407 & 0.1686 & 0.0040 & 0.0137 & -0.0213 & 1.4325 & 0.0177 & -0.0154 & -7.4961 \\
\hline 2 & 0.2375 & 0.2282 & 0.1136 & 0.1071 & 0.1240 & 0.1211 & 0.0065 & 0.0028 & 0.0094 & 0.3596 & 0.0403 & 7.2835 & 1.0471 & 7.4505 & 0.5506 & 0.1853 & 0.0044 & 0.0187 & -0.0213 & 1.3708 & 0.0257 & -0.0227 & -6.4401 \\
\hline 3 & 0.2567 & 0.2493 & 0.1387 & 0.1327 & 0.1180 & 0.1166 & 0.0060 & 0.0014 & 0.0074 & 0.5331 & 0.0341 & 7.9018 & 1.2246 & 6.6274 & 0.5581 & 0.2598 & 0.0051 & 0.0253 & $\begin{array}{l}-0.0210 \\
-0.0249\end{array}$ & 1.2373 & 0.0346 & -0.0304 & $\begin{array}{l}-0.40101 \\
-5.5465\end{array}$ \\
\hline 4 & 0.2864 & 0.2823 & 0.1717 & 0.1675 & 0.1147 & 0.1148 & 0.0042 & -0.0001 & 0.0041 & 0.8136 & 0.0216 & 8.7067 & 1.2761 & 5.7649 & 0.5997 & 0.3071 & 0.0058 & 0.0338 & -0.0308 & 1.0491 & 0.0464 & & -4.4310 \\
\hline 5 & 0.3429 & 0.3449 & 0.2336 & 0.2347 & 0.1093 & 0.1102 & -0.0011 & -0.0010 & $\begin{array}{l}-0.0021 \\
-0.01\end{array}$ & 2.8115 & -0.0003 & 10.5156 & 1.0101 & 4.4547 & 0.7443 & 0.0910 & 0.0160 & 0.0548 & -0.0331 & 0.6286 & 0.0817 & -0.0645 & -2.4951 \\
\hline \multicolumn{24}{|c|}{ Panel E: Stocks Sorted by RSK } \\
\hline Quintile & RVJP & RVJN & RVLJP & RVLJN & RVSJP & RVSJN & SRVLJ & SRVSJ & SRVJ & RVOL & RSK & RKT & BETA & $\log ($ Size) & BEME & MOM & REV & IVOL & CSK & CKT & MAX & MIN & ILLIQ \\
\hline 1 & 0.2077 & 0.3914 & 0.1200 & 0.2822 & 0.0877 & 0.1091 & -0.1622 & -0.0215 & -0.1837 & 0.9182 & -0.9829 & 10.3573 & 1.0293 & 6.1615 & 0.6212 & 0.2118 & -0.0274 & 0.0312 & -0.0611 & 1.0555 & 0.0324 & -0.0472 & -4.7275 \\
\hline 2 & 0.2203 & 0.2758 & 0.0967 & 0.1366 & 0.1235 & 0.1393 & -0.0398 & -0.0157 & -0.0556 & 0.9332 & -0.2596 & 6.8740 & $\begin{array}{l}1.1303 \\
\text { n }\end{array}$ & 6.7033 & 0.5719 & 0.2052 & -0.0077 & 0.0291 & -0.0412 & $\begin{array}{l}1.1981 \\
\text { n }\end{array}$ & 0.0353 & -0.0374 & -5.5757 \\
\hline 3 & 0.2430 & 0.2394 & 0.1057 & 0.1035 & 0.1373 & 0.1359 & 0.0023 & 0.0014 & 0.0037 & $\begin{array}{l}1.1222 \\
\end{array}$ & 0.0189 & 6.5111 & 1.1288 & 6.8070 & 0.5748 & 0.1932 & 0.0073 & 0.0283 & -0.0238 & 1.2155 & 0.0391 & $\begin{array}{l}-0.0325 \\
\end{array}$ & -5.7121 \\
\hline 4 & 0.2786 & 0.2142 & 0.1418 & 0.0960 & 0.1368 & 0.1182 & 0.0459 & 0.0186 & 0.0644 & 0.8769 & 0.3040 & 6.9460 & 1.1139 & 6.7671 & 0.5790 & 0.2056 & 0.0231 & 0.0280 & -0.0098 & 1.2017 & 0.0444 & -0.0290 & -5.6422 \\
\hline 5 & 0.3996 & 0.1978 & 0.2947 & 0.1179 & 0.1049 & 0.0800 & 0.1769 & 0.0249 & 0.2018 & 0.8942 & 1.0656 & 10.5964 & 0.9944 & 6.2012 & 0.6374 & 0.1963 & 0.0400 & 0.0297 & 0.0045 & 1.0482 & 0.0549 & -0.0272 & -4.7552 \\
\hline \multicolumn{24}{|c|}{ Panel F: Stocks Sorted by RKT } \\
\hline Quintile & RVJP & RVJN & RVLJP & RVLJN & RVSJP & RVSJN & SRVLJ & SRVSJ & SRVJ & RVOL & RSK & RKT & BETA & $\log ($ Size) & BEME & MOM & REV & IVOL & CSK & CKT & MAX & MIN & ILLIQ \\
\hline 1 & 0.1804 & 0.1785 & 0.0257 & 0.0248 & 0.1548 & 0.1536 & 0.0008 & 0.0011 & 0.0019 & 0.6884 & 0.0110 & 4.4470 & 1.1920 & 7.6130 & 0.5303 & 0.1864 & 0.0054 & 0.0248 & -0.0222 & 1.3529 & 0.0339 & -0.0290 & -6.7592 \\
\hline 2 & 0.2242 & 0.2206 & 0.0757 & 0.0738 & 0.1484 & 0.1468 & 0.0020 & 0.0017 & 0.0036 & 0.7285 & 0.0167 & 5.7679 & 1.1586 & 6.9411 & 0.5522 & 0.2203 & 0.0077 & 0.0276 & -0.0252 & 1.2505 & 0.0387 & -0.0324 & -5.9541 \\
\hline 3 & 0.2630 & 0.2582 & 0.1340 & 0.1310 & 0.1291 & 0.1272 & 0.0030 & 0.0019 & 0.0048 & 0.8130 & 0.0215 & 7.0711 & 1.1103 & 6.5028 & 0.5817 & 0.2175 & 0.0083 & 0.0296 & -0.0265 & 1.1627 & 0.0417 & -0.0347 & -5.3464 \\
\hline 4 & 0.3070 & 0.3004 & 0.2067 & 0.2017 & 0.1004 & 0.0987 & 0.0049 & 0.0017 & 0.0066 & 0.9433 & 0.0292 & 8.9744 & $\begin{array}{l}1.10438 \\
1.048\end{array}$ & $\begin{array}{l}.0 .0208 \\
6.0841\end{array}$ & 0.6186 & 0.2085 & 0.0076 & 0.0313 & $\begin{array}{l}-0.0203 \\
-0.0281\end{array}$ & $\begin{array}{l}1.1067 \\
1.0644\end{array}$ & 0.0440 & $\begin{array}{l}-0.0547 \\
-0.0370\end{array}$ & $\begin{array}{l}-5.530404 \\
-4.6922\end{array}$ \\
\hline 5 & 0.3745 & 0.3612 & 0.3171 & 0.3051 & 0.0574 & 0.0561 & 0.0119 & 0.0013 & 0.0133 & 1.5722 & 0.0660 & 15.0322 & 0.8918 & 5.4974 & 0.7022 & 0.1793 & 0.0064 & 0.0332 & -0.0295 & 0.8881 & 0.0478 & -0.0403 & -3.6585 \\
\hline
\end{tabular}




\section{Empirical Results}

In this section, we begin by discussing single (univariate) portfolio sort results (see Tables 4-7), and double sort results (see Tables 8-13). We then discuss results based on a firm-level Fama-MacBeth regression analysis and an analysis of jumps and news announcements. We assume a weekly holding period, and return calculations reported in the tables are carried out as follows. At the end of each Tuesday, stocks are sorted into quintile portfolios based on different realized variation measures (see Panel A of Table 1). We then calculate equal-weighted and value-weighted portfolio returns over the subsequent week. We report the time series average of these weekly returns for each portfolio (these returns are called "Mean Return" in the tables). In addition, we regress the excess return of each portfolio on the Fama-French and Carhart (FFC4) factors to control for systematic risks, using regression of the form

$$
r_{i, t}-r_{f, t}=\alpha_{i}+\beta_{i}^{M K T}\left(M K T_{t}-r_{f, t}\right)+\beta_{i}^{S M B} S M B_{t}+\beta_{i}^{H M L} H M L_{t}+\beta_{i}^{U M D} U M D_{t}+\epsilon_{i, t}
$$

where $r_{i, t}$ denotes the weekly return for firm i, $r_{f, t}$ is the risk-free rate; and $M K T_{t}, S M B_{t}, H M L_{t}$, and $U M D_{t}$ denote FFC4 market, size, value and momentum factors, respectively. The intercepts from these regressions (called "Alpha" in our tabulated results), measure risk-adjusted excess returns, and are also reported in Tables 4-13. Needless to say, our objective in these tables is to assess whether predictability exists, after controlling for various systematic risk factors. Finally, in Table 14, we report the results of cross-sectional (firm-level) Fama-MacBeth regressions used to investigate return predictability when simultaneously controlling for multiple realized measures and firm characteristics. 
Table 4. Univariate Portfolio Sorts Based on Positive, Negative, and Signed Total Jump Variation.

\begin{tabular}{lccccccccccccc}
\hline \multicolumn{10}{l}{ Panel A: Stocks Sorted by RVJP } \\
\hline \multicolumn{10}{c}{ Equal-Weighted Returns and Alphas } \\
\hline Quintile & $\mathbf{1}$ (Low) & $\mathbf{2}$ & $\mathbf{3}$ & $\mathbf{4}$ & $\mathbf{5}$ (High) & High-Low & $\mathbf{1}$ (Low) & $\mathbf{2}$ & $\mathbf{3}$ & $\mathbf{4}$ & $\mathbf{5}$ (High) & High-Low \\
\hline Mean Return & 33.65 & 30.50 & 33.04 & 28.08 & 20.64 & $-13.01^{* * *}$ & 23.52 & 19.48 & 17.93 & 20.91 & 18.27 & -5.25 \\
& $(3.54)$ & $(3.28)$ & $(3.49)$ & $(2.87)$ & $(2.19)$ & $(-2.75)$ & $(3.54)$ & $(3.27)$ & $(2.93)$ & $(3.35)$ & $(2.83)$ & $(-1.35)$ \\
Alpha & 10.59 & 7.47 & 11.24 & 7.52 & 2.88 & -7.71 & 2.88 & -0.63 & -2.30 & 0.67 & -2.75 & $-5.63 *$ \\
& $(4.16)$ & $(3.64)$ & $(4.34)$ & $(2.33)$ & $(0.72)$ & $(-1.64)$ & $(2.31)$ & $(-0.44)$ & $(-1.22)$ & $(0.32)$ & $(-1.19)$ & $(-1.87)$ \\
\hline
\end{tabular}

Panel B: Stocks Sorted by RVJN

\begin{tabular}{|c|c|c|c|c|c|c|c|c|c|c|c|c|}
\hline \multirow[b]{2}{*}{ Quintile } & \multicolumn{6}{|c|}{ Equal-Weighted Returns and Alphas } & \multicolumn{6}{|c|}{ Value-Weighted Returns and Alphas } \\
\hline & 1 (Low) & 2 & 3 & 4 & 5 (High) & High-Low & 1 (Low) & 2 & 3 & 4 & 5 (High) & High-Low \\
\hline Mean Return & $\begin{array}{l}13.17 \\
(1.52)\end{array}$ & $\begin{array}{l}23.01 \\
(2.59)\end{array}$ & $\begin{array}{l}26.77 \\
(2.84)\end{array}$ & $\begin{array}{l}33.79 \\
(3.36)\end{array}$ & $\begin{array}{l}49.23 \\
(4.62)\end{array}$ & $\begin{array}{c}36.06^{* * *} \\
(6.47)\end{array}$ & $\begin{array}{l}16.23 \\
(2.55)\end{array}$ & $\begin{array}{l}25.44 \\
(4.11)\end{array}$ & $\begin{array}{l}26.41 \\
(4.08)\end{array}$ & $\begin{array}{l}26.62 \\
(3.93)\end{array}$ & $\begin{array}{l}31.36 \\
(4.29)\end{array}$ & $\begin{array}{c}15.13^{* * *} \\
(3.75)\end{array}$ \\
\hline Alpha & $\begin{array}{l}-9.36 \\
(-4.46)\end{array}$ & $\begin{array}{c}-0.03 \\
(-0.02)\end{array}$ & $\begin{array}{c}4.18 \\
(1.86)\end{array}$ & $\begin{array}{l}13.24 \\
(3.93)\end{array}$ & $\begin{array}{l}31.71 \\
(6.34)\end{array}$ & $\begin{array}{c}41.07^{* * *} \\
(7.51)\end{array}$ & $\begin{array}{l}-3.55 \\
(-3.08)\end{array}$ & $\begin{array}{c}4.94 \\
(3.02)\end{array}$ & $\begin{array}{c}5.27 \\
(2.64)\end{array}$ & $\begin{array}{c}5.49 \\
(2.37)\end{array}$ & $\begin{array}{l}10.05 \\
(4.13)\end{array}$ & $\begin{array}{c}13.60 * * * \\
(4.52)\end{array}$ \\
\hline
\end{tabular}

Panel C: Stocks Sorted by SRVJ

\begin{tabular}{lccccccccccccc}
\hline \multicolumn{10}{l|c}{ Panel C: Stocks Sorted by SRVJ } \\
\hline Quintile & $\mathbf{1}$ (Low) & $\mathbf{2}$ & $\mathbf{3}$ & $\mathbf{4}$ & $\mathbf{5}$ (High) & High-Low & $\mathbf{1}$ (Low) & $\mathbf{2}$ & $\mathbf{3}$ & $\mathbf{4}$ & $\mathbf{5}$ (High) & High-Low \\
\hline Mean Return & 51.85 & 39.02 & 26.15 & 17.86 & 11.02 & $-40.82^{* * *}$ & 34.67 & 27.43 & 19.93 & 13.64 & 9.65 & $-25.02 * * *$ \\
& $(5.14)$ & $(3.85)$ & $(2.70)$ & $(1.98)$ & $(1.33)$ & $(-9.85)$ & $(4.85)$ & $(4.12)$ & $(3.10)$ & $(2.16)$ & $(1.59)$ & $(-5.78)$ \\
Alpha & 30.54 & 17.81 & 4.56 & -3.58 & -9.64 & $-40.18^{* * *}$ & 13.44 & 6.94 & -0.52 & -6.53 & -10.25 & $-23.69 * *$ \\
& $(8.40)$ & $(5.78)$ & $(1.74)$ & $(-1.56)$ & $(-4.05)$ & $(-10.10)$ & $(5.01)$ & $(3.95)$ & $(-0.40)$ & $(-4.48)$ & $(-4.47)$ & $(-5.56)$ \\
\hline
\end{tabular}

Notes: Entries in this table are average returns and risk-adjusted alphas for single-sorted portfolios based on RVJP, RVJN and SRVJ, which are described in Table 2. The sample includes all NYSE, NASDAQ and AMEX listed stocks for the period January 1993 to December 2016. At the end of each Tuesday, all the stocks in the sample are sorted into quintile portfolios based on ascending values of the various jump variation measures listed in the title of each panel. Each portfolio is held for one week. The row labeled "Mean Return" reports the time series average values of one-week-ahead equal-weighted and value-weighted returns for quintile portfolios. The row labeled "Alpha" reports Fama-French-Carhart four-factor alphas, based on the model (14), for each of the quintile portfolios, as well as for the difference between portfolio 5 and portfolio 1 . Newey-West $t$-statistics are given in parentheses; and $* * *$, and ${ }^{* * *}$ denote means and alphas that are significant at the $10 \%, 5 \%$, and $1 \%$ levels, respectively. 
Table 5. Univariate Portfolio Sorts Based on Positive, Negative, and Signed Large Jump Variation.

\begin{tabular}{|c|c|c|c|c|c|c|c|c|c|c|c|c|}
\hline \multicolumn{13}{|c|}{ Panel A: Stocks Sorted by RVLJP } \\
\hline & \multicolumn{6}{|c|}{ Equal-Weighted Returns and Alphas } & \multicolumn{6}{|c|}{ Value-Weighted Returns and Alphas } \\
\hline Quintile & 1 (Low) & 2 & 3 & 4 & 5 (High) & High-Low & 1 (Low) & 2 & 3 & 4 & 5 (High) & High-Low \\
\hline \multicolumn{13}{|c|}{ Part I: Jump Truncation Level $=\gamma^{1}$} \\
\hline Mean Return & $\begin{array}{l}31.40 \\
(3.31)\end{array}$ & $\begin{array}{l}30.00 \\
(3.19)\end{array}$ & $\begin{array}{l}29.17 \\
(3.10)\end{array}$ & $\begin{array}{l}31.04 \\
(3.28)\end{array}$ & $\begin{array}{l}23.78 \\
(2.48)\end{array}$ & $\begin{array}{l}-7.61 * * \\
(-2.05)\end{array}$ & $\begin{array}{l}21.78 \\
(3.41)\end{array}$ & $\begin{array}{l}20.61 \\
(3.21)\end{array}$ & $\begin{array}{l}20.72 \\
(3.33)\end{array}$ & $\begin{array}{l}18.56 \\
(2.95)\end{array}$ & $\begin{array}{l}17.75 \\
(2.66)\end{array}$ & $\begin{array}{c}-4.03 \\
(-1.09)\end{array}$ \\
\hline Alpha & $\begin{array}{l}10.07 \\
(4.20)\end{array}$ & $\begin{array}{c}7.64 \\
(3.44)\end{array}$ & $\begin{array}{c}6.65 \\
(2.81)\end{array}$ & $\begin{array}{c}9.04 \\
(3.35)\end{array}$ & $\begin{array}{c}6.08 \\
(1.53)\end{array}$ & $\begin{array}{l}-3.98 \\
(-1.08)\end{array}$ & $\begin{array}{c}1.97 \\
(1.95)\end{array}$ & $\begin{array}{c}0.68 \\
(0.52)\end{array}$ & $\begin{array}{c}-0.42 \\
(-0.26)\end{array}$ & $\begin{array}{c}-2.32 \\
(-1.27)\end{array}$ & $\begin{array}{c}-2.83 \\
(-1.21)\end{array}$ & $\begin{array}{l}-4.80 * \\
(-1.71)\end{array}$ \\
\hline \multicolumn{13}{|c|}{ Part II: Jump Truncation Level $=\gamma^{2}$} \\
\hline Mean Return & $\begin{array}{l}29.42 \\
(3.15)\end{array}$ & $\begin{array}{l}43.86 \\
(2.16)\end{array}$ & $\begin{array}{l}30.00 \\
(3.04)\end{array}$ & $\begin{array}{l}29.12 \\
(3.07)\end{array}$ & $\begin{array}{l}25.77 \\
(2.74)\end{array}$ & $\begin{array}{c}-3.65 \\
(-1.11)\end{array}$ & $\begin{array}{l}20.42 \\
(3.25)\end{array}$ & $\begin{array}{l}27.59 \\
(1.77)\end{array}$ & $\begin{array}{l}20.12 \\
(2.98)\end{array}$ & $\begin{array}{l}22.50 \\
(3.63)\end{array}$ & $\begin{array}{l}20.39 \\
(3.14)\end{array}$ & $\begin{array}{c}-0.03 \\
(-0.01)\end{array}$ \\
\hline Alpha & $\begin{array}{c}7.74 \\
(3.70)\end{array}$ & $\begin{array}{l}32.93 \\
(3.23)\end{array}$ & $\begin{array}{c}7.28 \\
(2.92)\end{array}$ & $\begin{array}{c}7.02 \\
(2.62)\end{array}$ & $\begin{array}{l}7.70 \\
(2.02)\end{array}$ & $\begin{array}{c}-0.04 \\
(-0.01)\end{array}$ & $\begin{array}{c}0.45 \\
(0.72)\end{array}$ & $\begin{array}{l}13.80 \\
(1.35)\end{array}$ & $\begin{array}{c}-0.27 \\
(-0.13)\end{array}$ & $\begin{array}{c}1.60 \\
(0.83)\end{array}$ & $\begin{array}{c}-0.52 \\
(-0.22)\end{array}$ & $\begin{array}{c}-0.97 \\
(-0.37)\end{array}$ \\
\hline \multicolumn{13}{|c|}{ Panel B: Stocks Sorted by RVLJN } \\
\hline & \multicolumn{6}{|c|}{ Equal-Weighted Returns and Alphas } & \multicolumn{6}{|c|}{ Value-Weighted Returns and Alphas } \\
\hline Quintile & 1 (Low) & 2 & 3 & 4 & 5 (High) & High-Low & 1 (Low) & 2 & 3 & 4 & 5 (High) & High-Low \\
\hline \multicolumn{13}{|c|}{ Part I: Jump Truncation Level $=\gamma^{1}$} \\
\hline Mean Return & $\begin{array}{l}23.80 \\
(2.65)\end{array}$ & $\begin{array}{l}23.68 \\
(2.59)\end{array}$ & $\begin{array}{l}27.64 \\
(2.98)\end{array}$ & $\begin{array}{l}28.85 \\
(2.95)\end{array}$ & $\begin{array}{l}41.56 \\
(4.10)\end{array}$ & $\begin{array}{c}17.76^{* * *} \\
(4.47)\end{array}$ & $\begin{array}{l}19.65 \\
(3.10)\end{array}$ & $\begin{array}{l}19.58 \\
(3.10)\end{array}$ & $\begin{array}{l}25.63 \\
(4.00)\end{array}$ & $\begin{array}{l}22.38 \\
(3.27)\end{array}$ & $\begin{array}{l}25.24 \\
(3.44)\end{array}$ & $\begin{array}{c}5.59 \\
(1.41)\end{array}$ \\
\hline Alpha & $\begin{array}{c}2.31 \\
(1.11)\end{array}$ & $\begin{array}{c}1.11 \\
(0.55)\end{array}$ & $\begin{array}{c}5.00 \\
(2.32)\end{array}$ & $\begin{array}{c}7.10 \\
(2.47)\end{array}$ & $\begin{array}{l}23.74 \\
(5.39)\end{array}$ & $\begin{array}{c}21.43^{* * *} \\
(5.53)\end{array}$ & $\begin{array}{c}-0.20 \\
(-0.18)\end{array}$ & $\begin{array}{l}-1.32 \\
(-0.92)\end{array}$ & $\begin{array}{c}4.52 \\
(2.47)\end{array}$ & $\begin{array}{c}0.89 \\
(0.40)\end{array}$ & $\begin{array}{c}4.34 \\
(1.82)\end{array}$ & $\begin{array}{c}4.54 \\
(1.61)\end{array}$ \\
\hline \multicolumn{13}{|c|}{ Part II: Jump Truncation Level $=\gamma^{2}$} \\
\hline Mean Return & $\begin{array}{l}24.76 \\
(2.71)\end{array}$ & $\begin{array}{c}6.46 \\
(0.33)\end{array}$ & $\begin{array}{l}27.89 \\
(2.75)\end{array}$ & $\begin{array}{l}29.12 \\
(3.03)\end{array}$ & $\begin{array}{l}38.62 \\
(3.88)\end{array}$ & $\begin{array}{c}13.86^{* * *} \\
(4.01)\end{array}$ & $\begin{array}{l}19.69 \\
(3.13)\end{array}$ & $\begin{array}{l}13.21 \\
(0.93)\end{array}$ & $\begin{array}{l}21.46 \\
(3.05)\end{array}$ & $\begin{array}{l}23.20 \\
(3.61)\end{array}$ & $\begin{array}{l}22.34 \\
(3.15)\end{array}$ & $\begin{array}{c}2.66 \\
(0.75)\end{array}$ \\
\hline Alpha & $\begin{array}{c}3.02 \\
(1.63)\end{array}$ & $\begin{array}{c}6.75 \\
(1.08)\end{array}$ & $\begin{array}{c}6.58 \\
(2.50)\end{array}$ & $\begin{array}{c}7.06 \\
(2.67)\end{array}$ & $\begin{array}{l}20.62 \\
(4.81)\end{array}$ & $\begin{array}{c}17.60^{* * *} \\
(5.06)\end{array}$ & $\begin{array}{c}-0.30 \\
(-0.45)\end{array}$ & $\begin{array}{c}6.52 \\
(0.91)\end{array}$ & $\begin{array}{c}1.26 \\
(0.57)\end{array}$ & $\begin{array}{c}2.28 \\
(1.06)\end{array}$ & $\begin{array}{c}1.58 \\
(0.66)\end{array}$ & $\begin{array}{c}1.88 \\
(0.70)\end{array}$ \\
\hline
\end{tabular}


Table 5. Cont.

\begin{tabular}{|c|c|c|c|c|c|c|c|c|c|c|c|c|}
\hline \multicolumn{13}{|c|}{ Panel C: Stocks Sorted by SRVLJ } \\
\hline \multirow[b]{2}{*}{ Quintile } & \multicolumn{6}{|c|}{ Equal-Weighted Returns and Alphas } & \multicolumn{6}{|c|}{ Value-Weighted Returns and Alphas } \\
\hline & 1 (Low) & 2 & 3 & 4 & 5 (High) & High-Low & 1 (Low) & 2 & 3 & 4 & 5 (High) & High-Low \\
\hline \multicolumn{13}{|c|}{ Part I: Jump Truncation Level $=\gamma^{1}$} \\
\hline Mean Return & $\begin{array}{l}44.35 \\
(4.52)\end{array}$ & $\begin{array}{l}32.94 \\
(3.36)\end{array}$ & $\begin{array}{l}31.08 \\
(3.13)\end{array}$ & $\begin{array}{l}22.72 \\
(2.44)\end{array}$ & $\begin{array}{l}16.04 \\
(1.88)\end{array}$ & $\begin{array}{c}-28.31 \text { *** } \\
(-9.00)\end{array}$ & $\begin{array}{l}26.27 \\
(3.92)\end{array}$ & $\begin{array}{l}22.99 \\
(3.58)\end{array}$ & $\begin{array}{l}22.36 \\
(3.29)\end{array}$ & $\begin{array}{l}17.77 \\
(2.79)\end{array}$ & $\begin{array}{l}16.27 \\
(2.71)\end{array}$ & $\begin{array}{c}-10.01^{* * *} \\
(-3.09)\end{array}$ \\
\hline Alpha & $\begin{array}{l}23.47 \\
(7.13)\end{array}$ & $\begin{array}{l}11.38 \\
(4.20)\end{array}$ & $\begin{array}{c}8.91 \\
(3.04)\end{array}$ & $\begin{array}{c}0.96 \\
(0.40)\end{array}$ & $\begin{array}{c}-4.90 \\
(-2.17)\end{array}$ & $\begin{array}{c}-28.36^{* * *} \\
(-9.39)\end{array}$ & $\begin{array}{c}5.00 \\
(2.24)\end{array}$ & $\begin{array}{c}2.42 \\
(1.64)\end{array}$ & $\begin{array}{c}1.83 \\
(1.01)\end{array}$ & $\begin{array}{c}-2.64 \\
(-1.82)\end{array}$ & $\begin{array}{c}-4.26 \\
(-2.24)\end{array}$ & $\begin{array}{c}-9.25^{* * * *} \\
(-2.87)\end{array}$ \\
\hline \multicolumn{13}{|c|}{ Part II: Jump Truncation Level $=\gamma^{2}$} \\
\hline Mean Return & $\begin{array}{l}40.55 \\
(4.19)\end{array}$ & $\begin{array}{l}28.37 \\
(2.91)\end{array}$ & $\begin{array}{l}33.05 \\
(1.48)\end{array}$ & $\begin{array}{l}24.16 \\
(2.55)\end{array}$ & $\begin{array}{l}19.03 \\
(2.19)\end{array}$ & $\begin{array}{c}-21.52^{* * *} \\
(-8.22)\end{array}$ & $\begin{array}{l}22.59 \\
(3.40)\end{array}$ & $\begin{array}{l}20.48 \\
(3.18)\end{array}$ & $\begin{array}{l}16.45 \\
(1.14)\end{array}$ & $\begin{array}{l}18.86 \\
(3.02)\end{array}$ & $\begin{array}{l}20.14 \\
(3.27)\end{array}$ & $\begin{array}{l}-2.45 \\
(-0.80)\end{array}$ \\
\hline Alpha & $\begin{array}{l}19.59 \\
(6.15)\end{array}$ & $\begin{array}{c}8.18 \\
(3.29)\end{array}$ & $\begin{array}{l}24.26 \\
(2.16)\end{array}$ & $\begin{array}{c}2.23 \\
(0.82)\end{array}$ & $\begin{array}{l}-1.97 \\
(-0.84)\end{array}$ & $\begin{array}{c}-21.55^{* * *} \\
(-8.33)\end{array}$ & $\begin{array}{c}1.82 \\
(0.86)\end{array}$ & $\begin{array}{c}0.76 \\
(0.68)\end{array}$ & $\begin{array}{l}6.85 \\
(1.04)\end{array}$ & $\begin{array}{l}-2.41 \\
(-1.23)\end{array}$ & $\begin{array}{l}-0.26 \\
(-0.13)\end{array}$ & $\begin{array}{l}-2.08 \\
(-0.69)\end{array}$ \\
\hline
\end{tabular}

Notes: See notes to Table 4. Entries are average returns and risk-adjusted alphas for single-sorted portfolios based on RVLJP, RVLJN and SRVLJ. Jump truncation levels are $\gamma^{1}=4 \sqrt{\frac{1}{t} \widehat{I V}_{t}^{(i)}} \Delta_{n}^{0.49}$ and $\gamma^{2}=5 \sqrt{\frac{1}{t} \widehat{I V}_{t}^{(i)}} \Delta_{n}^{0.49}$.

Table 6. Univariate Portfolio Sorts Based on Positive, Negative, and Signed Small Jump Variation.

\begin{tabular}{|c|c|c|c|c|c|c|c|c|c|c|c|c|}
\hline \multicolumn{13}{|c|}{ Panel A: Stocks Sorted by RVSJP } \\
\hline & \multicolumn{6}{|c|}{ Equal-Weighted Returns and Alphas } & \multicolumn{6}{|c|}{ Value-Weighted Returns and Alphas } \\
\hline Quintile & 1 (Low) & 2 & 3 & 4 & 5 (High) & High-Low & 1 (Low) & 2 & 3 & 4 & 5 (High) & High-Low \\
\hline \multicolumn{13}{|c|}{ Part I: Jump Truncation Level $=\gamma^{1}$} \\
\hline Mean Return & $\begin{array}{l}32.01 \\
(3.44)\end{array}$ & $\begin{array}{l}32.51 \\
(3.43)\end{array}$ & $\begin{array}{l}29.03 \\
(3.09)\end{array}$ & $\begin{array}{l}26.59 \\
(2.85)\end{array}$ & $\begin{array}{l}25.72 \\
(2.65)\end{array}$ & $\begin{array}{l}-6.29 * * \\
(-2.14)\end{array}$ & $\begin{array}{l}27.93 \\
(3.65)\end{array}$ & $\begin{array}{l}26.10 \\
(3.84)\end{array}$ & $\begin{array}{l}18.44 \\
(2.91)\end{array}$ & $\begin{array}{l}16.52 \\
(2.75)\end{array}$ & $\begin{array}{l}15.37 \\
(2.45)\end{array}$ & $\begin{array}{c}-12.55^{* *} \\
(-2.54)\end{array}$ \\
\hline Alpha & $\begin{array}{l}13.11 \\
(3.74)\end{array}$ & $\begin{array}{c}9.39 \\
(4.21)\end{array}$ & $\begin{array}{c}6.37 \\
(3.02)\end{array}$ & $\begin{array}{c}4.77 \\
(2.08)\end{array}$ & $\begin{array}{c}6.13 \\
(1.82)\end{array}$ & $\begin{array}{c}-6.98^{* * *} \\
(-2.65)\end{array}$ & $\begin{array}{l}7.40 \\
(2.44)\end{array}$ & $\begin{array}{c}5.21 \\
(3.28)\end{array}$ & $\begin{array}{l}-1.83 \\
(-1.39)\end{array}$ & $\begin{array}{c}-2.89 \\
(-1.55)\end{array}$ & $\begin{array}{c}-4.95 \\
(-2.32)\end{array}$ & $\begin{array}{c}-12.35^{* * *} \\
(-2.93)\end{array}$ \\
\hline \multicolumn{13}{|c|}{ Part II: Jump Truncation Level $=\gamma^{2}$} \\
\hline Mean Return & $\begin{array}{l}34.25 \\
(3.72)\end{array}$ & $\begin{array}{l}31.55 \\
(3.37)\end{array}$ & $\begin{array}{l}28.43 \\
(3.04)\end{array}$ & $\begin{array}{l}27.41 \\
(2.90)\end{array}$ & $\begin{array}{l}24.23 \\
(2.48)\end{array}$ & $\begin{array}{c}-10.02 * * * \\
(-3.26)\end{array}$ & $\begin{array}{l}29.37 \\
(4.09)\end{array}$ & $\begin{array}{l}18.76 \\
(2.95)\end{array}$ & $\begin{array}{l}17.76 \\
(2.94)\end{array}$ & $\begin{array}{l}14.75 \\
(2.39)\end{array}$ & $\begin{array}{l}18.98 \\
(2.93)\end{array}$ & $\begin{array}{c}-10.40 * * \\
(-2.25)\end{array}$ \\
\hline Alpha & $\begin{array}{l}14.08 \\
(4.93)\end{array}$ & $\begin{array}{c}8.85 \\
(4.12)\end{array}$ & $\begin{array}{c}5.90 \\
(2.73)\end{array}$ & $\begin{array}{c}5.75 \\
(2.32)\end{array}$ & $\begin{array}{c}5.08 \\
(1.42)\end{array}$ & $\begin{array}{c}-9.00 * * * \\
(-3.13)\end{array}$ & $\begin{array}{c}8.52 \\
(3.87)\end{array}$ & $\begin{array}{l}-2.00 \\
(-1.52)\end{array}$ & $\begin{array}{l}-2.01 \\
(-1.24)\end{array}$ & $\begin{array}{l}-5.30 \\
(-2.67)\end{array}$ & $\begin{array}{c}-1.51 \\
(-0.62)\end{array}$ & $\begin{array}{c}-10.02 * * \\
(-2.54)\end{array}$ \\
\hline
\end{tabular}


Table 6. Cont.

\begin{tabular}{|c|c|c|c|c|c|c|c|c|c|c|c|c|}
\hline \multicolumn{13}{|c|}{ Panel B: Stocks Sorted by RVSJN } \\
\hline \multirow[b]{2}{*}{ Quintile } & \multicolumn{6}{|c|}{ Equal-Weighted Returns and Alphas } & \multicolumn{6}{|c|}{ Value-Weighted Returns and Alphas } \\
\hline & 1 (Low) & 2 & 3 & 4 & 5 (High) & High-Low & 1 (Low) & 2 & 3 & 4 & 5 (High) & High-Low \\
\hline \multicolumn{13}{|c|}{ Part I: Jump Truncation Level $=\gamma^{1}$} \\
\hline Mean Return & $\begin{array}{l}23.66 \\
(2.64)\end{array}$ & $\begin{array}{l}21.60 \\
(2.38)\end{array}$ & $\begin{array}{l}27.21 \\
(2.97)\end{array}$ & $\begin{array}{l}31.73 \\
(3.35)\end{array}$ & $\begin{array}{l}41.77 \\
(3.93)\end{array}$ & $\begin{array}{c}18.10^{* * *} \\
(5.07)\end{array}$ & $\begin{array}{c}6.94 \\
(1.00)\end{array}$ & $\begin{array}{l}15.90 \\
(2.42)\end{array}$ & $\begin{array}{l}21.97 \\
(3.46)\end{array}$ & $\begin{array}{l}27.77 \\
(4.34)\end{array}$ & $\begin{array}{l}31.26 \\
(4.68)\end{array}$ & $\begin{array}{c}24.32 * * * \\
(5.00)\end{array}$ \\
\hline Alpha & $\begin{array}{c}5.26 \\
(1.59)\end{array}$ & $\begin{array}{c}-1.04 \\
(-0.51)\end{array}$ & $\begin{array}{c}4.35 \\
(2.22)\end{array}$ & $\begin{array}{c}9.56 \\
(4.02)\end{array}$ & $\begin{array}{l}21.62 \\
(5.49)\end{array}$ & $\begin{array}{c}16.36^{* * *} \\
(5.46)\end{array}$ & $\begin{array}{l}-13.39 \\
(-4.54)\end{array}$ & $\begin{array}{c}-4.28 \\
(-2.80)\end{array}$ & $\begin{array}{c}1.80 \\
(1.33)\end{array}$ & $\begin{array}{c}7.41 \\
(3.72)\end{array}$ & $\begin{array}{l}10.52 \\
(4.07)\end{array}$ & $\begin{array}{c}23.91 * * * \\
(5.21)\end{array}$ \\
\hline \multicolumn{13}{|c|}{ Part II: Jump Truncation Level $=\gamma^{2}$} \\
\hline Mean Return & $\begin{array}{l}19.42 \\
(2.23)\end{array}$ & $\begin{array}{l}23.04 \\
(2.60)\end{array}$ & $\begin{array}{l}26.65 \\
(2.93)\end{array}$ & $\begin{array}{l}32.48 \\
(3.35)\end{array}$ & $\begin{array}{l}44.37 \\
(4.09)\end{array}$ & $\begin{array}{c}24.96^{* * * *} \\
(6.07)\end{array}$ & $\begin{array}{l}14.22 \\
(2.13)\end{array}$ & $\begin{array}{l}18.78 \\
(3.00)\end{array}$ & $\begin{array}{l}25.82 \\
(4.13)\end{array}$ & $\begin{array}{l}29.05 \\
(4.42)\end{array}$ & $\begin{array}{l}32.60 \\
(4.43)\end{array}$ & $\begin{array}{c}18.38^{* * *} \\
(3.80)\end{array}$ \\
\hline Alpha & $\begin{array}{c}-0.37 \\
(-0.14)\end{array}$ & $\begin{array}{c}0.70 \\
(0.37)\end{array}$ & $\begin{array}{c}4.18 \\
(2.18)\end{array}$ & $\begin{array}{l}10.38 \\
(4.02)\end{array}$ & $\begin{array}{l}24.84 \\
(5.85)\end{array}$ & $\begin{array}{c}25.22 * * * \\
(7.21)\end{array}$ & $\begin{array}{c}-5.47 \\
(-2.72)\end{array}$ & $\begin{array}{c}-1.28 \\
(-1.07)\end{array}$ & $\begin{array}{c}5.81 \\
(3.39)\end{array}$ & $\begin{array}{c}7.80 \\
(3.67)\end{array}$ & $\begin{array}{l}10.85 \\
(4.02)\end{array}$ & $\begin{array}{c}16.31 * * * \\
(4.15)\end{array}$ \\
\hline \multicolumn{13}{|c|}{ Panel C: Stocks Sorted by SRVSJ } \\
\hline & \multicolumn{6}{|c|}{ Equal-Weighted Returns and Alphas } & \multicolumn{6}{|c|}{ Value-Weighted Returns and Alphas } \\
\hline Quintile & 1 (Low) & 2 & 3 & 4 & 5 (High) & High-Low & 1 (Low) & 2 & 3 & 4 & 5 (High) & High-Low \\
\hline \multicolumn{13}{|c|}{ Part I: Jump Truncation Level $=\gamma^{1}$} \\
\hline Mean Return & $\begin{array}{l}46.41 \\
(4.57)\end{array}$ & $\begin{array}{l}40.51 \\
(4.04)\end{array}$ & $\begin{array}{l}25.12 \\
(2.67)\end{array}$ & $\begin{array}{l}19.00 \\
(2.06)\end{array}$ & $\begin{array}{l}13.74 \\
(1.62)\end{array}$ & $\begin{array}{c}-32.67^{* * *} \\
(-8.60)\end{array}$ & $\begin{array}{l}34.54 \\
(5.00)\end{array}$ & $\begin{array}{l}24.08 \\
(3.58)\end{array}$ & $\begin{array}{l}18.26 \\
(2.81)\end{array}$ & $\begin{array}{l}17.84 \\
(2.85)\end{array}$ & $\begin{array}{l}10.42 \\
(1.72)\end{array}$ & $\begin{array}{c}-24.12 * * * \\
(-6.60)\end{array}$ \\
\hline Alpha & $\begin{array}{l}23.64 \\
(7.62)\end{array}$ & $\begin{array}{l}19.68 \\
(6.10)\end{array}$ & $\begin{array}{c}5.23 \\
(1.53)\end{array}$ & $\begin{array}{c}-2.09 \\
(-0.85)\end{array}$ & $\begin{array}{c}-8.14 \\
(-4.17)\end{array}$ & $\begin{array}{c}-31.78^{* * *} \\
(-9.01)\end{array}$ & $\begin{array}{l}13.77 \\
(6.18)\end{array}$ & $\begin{array}{c}3.25 \\
(1.91)\end{array}$ & $\begin{array}{l}-2.37 \\
(-1.07)\end{array}$ & $\begin{array}{l}-2.39 \\
(-1.52)\end{array}$ & $\begin{array}{c}-9.27 \\
(-5.00)\end{array}$ & $\begin{array}{c}-23.04^{* * *} \\
(-6.54)\end{array}$ \\
\hline \multicolumn{13}{|c|}{ Part II: Jump Truncation Level $=\gamma^{2}$} \\
\hline Mean Return & $\begin{array}{l}47.90 \\
(4.70)\end{array}$ & $\begin{array}{l}41.62 \\
(4.13)\end{array}$ & $\begin{array}{l}27.23 \\
(2.87)\end{array}$ & $\begin{array}{l}17.90 \\
(1.98)\end{array}$ & $\begin{array}{l}11.20 \\
(1.34)\end{array}$ & $\begin{array}{c}-36.70^{* * *} \\
(-9.06)\end{array}$ & $\begin{array}{l}36.88 \\
(5.31)\end{array}$ & $\begin{array}{l}25.13 \\
(3.79)\end{array}$ & $\begin{array}{l}18.45 \\
(2.86)\end{array}$ & $\begin{array}{l}14.98 \\
(2.39)\end{array}$ & $\begin{array}{c}9.41 \\
(1.52)\end{array}$ & $\begin{array}{c}-27.47^{* * *} \\
(-6.94)\end{array}$ \\
\hline Alpha & $\begin{array}{l}25.37 \\
(7.79)\end{array}$ & $\begin{array}{l}20.76 \\
(6.65)\end{array}$ & $\begin{array}{c}6.99 \\
(2.23)\end{array}$ & $\begin{array}{c}-3.03 \\
(-1.25)\end{array}$ & $\begin{array}{l}-10.51 \\
(-5.21)\end{array}$ & $\begin{array}{c}-35.88^{* * * *} \\
(-9.49)\end{array}$ & $\begin{array}{l}16.07 \\
(6.72)\end{array}$ & $\begin{array}{c}4.52 \\
(2.60)\end{array}$ & $\begin{array}{l}-1.87 \\
(-1.23)\end{array}$ & $\begin{array}{l}-5.39 \\
(-3.30)\end{array}$ & $\begin{array}{l}-10.34 \\
(-5.00)\end{array}$ & $\begin{array}{c}-26.41^{* * *} \\
(-6.72)\end{array}$ \\
\hline
\end{tabular}


Table 7. Univariate Portfolio Sorts Based on Realized Volatility, Skewness, Kurtosis and Continuous Variance.

\begin{tabular}{|c|c|c|c|c|c|c|c|c|c|c|c|c|}
\hline \multicolumn{13}{|c|}{ Panel A: Stocks Sorted by RVOL } \\
\hline \multirow[b]{2}{*}{ Quintile } & \multicolumn{6}{|c|}{ Equal-Weighted Returns and Alphas } & \multicolumn{6}{|c|}{ Value-Weighted Returns and Alphas } \\
\hline & 1 (Low) & 2 & 3 & 4 & 5 (High) & High-Low & 1 (Low) & 2 & 3 & 4 & 5 (High) & High-Low \\
\hline Mean Return & $\begin{array}{l}23.36 \\
(4.47)\end{array}$ & $\begin{array}{l}28.00 \\
(3.92)\end{array}$ & $\begin{array}{l}28.89 \\
(2.96)\end{array}$ & $\begin{array}{l}31.78 \\
(2.59)\end{array}$ & $\begin{array}{l}33.91 \\
(2.24)\end{array}$ & $\begin{array}{l}10.55 \\
(0.81)\end{array}$ & $\begin{array}{l}20.72 \\
(4.09)\end{array}$ & $\begin{array}{l}21.42 \\
(2.83)\end{array}$ & $\begin{array}{l}19.75 \\
(1.84)\end{array}$ & $\begin{array}{l}26.78 \\
(1.98)\end{array}$ & $\begin{array}{l}29.19 \\
(1.92)\end{array}$ & $\begin{array}{c}8.47 \\
(0.64)\end{array}$ \\
\hline Alpha & $\begin{array}{c}4.50 \\
(2.07)\end{array}$ & $\begin{array}{c}5.01 \\
(2.94)\end{array}$ & $\begin{array}{c}5.15 \\
(2.57)\end{array}$ & $\begin{array}{c}8.74 \\
(2.54)\end{array}$ & $\begin{array}{l}16.33 \\
(2.11)\end{array}$ & $\begin{array}{l}11.83 \\
(1.37)\end{array}$ & $\begin{array}{c}1.95 \\
(1.35)\end{array}$ & $\begin{array}{l}-1.39 \\
(-0.67)\end{array}$ & $\begin{array}{c}-3.94 \\
(-1.01)\end{array}$ & $\begin{array}{c}2.44 \\
(0.43)\end{array}$ & $\begin{array}{c}5.44 \\
(0.67)\end{array}$ & $\begin{array}{c}3.49 \\
(0.40)\end{array}$ \\
\hline \multicolumn{13}{|c|}{ Panel B: Stocks Sorted by RSK } \\
\hline & \multicolumn{6}{|c|}{ Equal-Weighted Returns and Alphas } & \multicolumn{6}{|c|}{ Value-Weighted Returns and Alphas } \\
\hline Quintile & 1 (Low) & 2 & 3 & 4 & 5 (High) & High-Low & 1 (Low) & 2 & 3 & 4 & 5 (High) & High-Low \\
\hline Mean Return & $\begin{array}{l}47.56 \\
(4.85)\end{array}$ & $\begin{array}{l}38.06 \\
(3.82)\end{array}$ & $\begin{array}{l}27.86 \\
(2.86)\end{array}$ & $\begin{array}{l}19.44 \\
(2.12)\end{array}$ & $\begin{array}{l}12.98 \\
(1.54)\end{array}$ & $\begin{array}{c}-34.58^{* * *} \\
(-9.94)\end{array}$ & $\begin{array}{l}29.45 \\
(4.27)\end{array}$ & $\begin{array}{l}27.52 \\
(4.22)\end{array}$ & $\begin{array}{l}19.27 \\
(2.98)\end{array}$ & $\begin{array}{l}14.68 \\
(2.32)\end{array}$ & $\begin{array}{l}13.21 \\
(2.18)\end{array}$ & $\begin{array}{c}-16.24^{* * *} \\
(-4.29)\end{array}$ \\
\hline Alpha & $\begin{array}{l}26.22 \\
(7.93)\end{array}$ & $\begin{array}{l}16.77 \\
(5.66)\end{array}$ & $\begin{array}{c}6.73 \\
(2.41)\end{array}$ & $\begin{array}{c}-2.15 \\
(-0.96)\end{array}$ & $\begin{array}{c}-7.90 \\
(-3.51)\end{array}$ & $\begin{array}{c}-34.12 * * * \\
(-10.08)\end{array}$ & $\begin{array}{c}7.87 \\
(3.30)\end{array}$ & $\begin{array}{c}7.02 \\
(4.44)\end{array}$ & $\begin{array}{c}-0.82 \\
(-0.60)\end{array}$ & $\begin{array}{l}-5.38 \\
(-3.73)\end{array}$ & $\begin{array}{c}-6.77 \\
(-3.23)\end{array}$ & $\begin{array}{c}-14.64^{* * *} \\
(-3.85)\end{array}$ \\
\hline \multicolumn{13}{|c|}{ Panel C: Stocks Sorted by RKT } \\
\hline & \multicolumn{6}{|c|}{ Equal-Weighted Returns and Alphas } & \multicolumn{6}{|c|}{ Value-Weighted Returns and Alphas } \\
\hline Quintile & 1 (Low) & 2 & 3 & 4 & 5 (High) & High-Low & 1 (Low) & 2 & 3 & 4 & 5 (High) & High-Low \\
\hline Mean Return & $\begin{array}{l}28.95 \\
(3.07)\end{array}$ & $\begin{array}{l}28.59 \\
(3.00)\end{array}$ & $\begin{array}{l}29.91 \\
(3.13)\end{array}$ & $\begin{array}{l}29.42 \\
(3.06)\end{array}$ & $\begin{array}{l}29.07 \\
(3.24)\end{array}$ & $\begin{array}{c}0.12 \\
(0.04)\end{array}$ & $\begin{array}{l}19.87 \\
(3.12)\end{array}$ & $\begin{array}{l}21.57 \\
(3.42)\end{array}$ & $\begin{array}{l}21.12 \\
(3.37)\end{array}$ & $\begin{array}{l}22.17 \\
(3.38)\end{array}$ & $\begin{array}{l}19.55 \\
(2.96)\end{array}$ & $\begin{array}{c}-0.32 \\
(-0.10)\end{array}$ \\
\hline Alpha & $\begin{array}{c}8.55 \\
(3.21)\end{array}$ & $\begin{array}{c}6.42 \\
(2.87)\end{array}$ & $\begin{array}{c}7.47 \\
(3.05)\end{array}$ & $\begin{array}{c}7.92 \\
(2.87)\end{array}$ & $\begin{array}{c}9.36 \\
(3.07)\end{array}$ & $\begin{array}{c}0.81 \\
(0.28)\end{array}$ & $\begin{array}{c}0.21 \\
(0.20)\end{array}$ & $\begin{array}{c}0.65 \\
(0.46)\end{array}$ & $\begin{array}{c}-0.10 \\
(-0.06)\end{array}$ & $\begin{array}{c}0.94 \\
(0.49)\end{array}$ & $\begin{array}{l}-1.92 \\
(-0.91)\end{array}$ & $\begin{array}{c}-2.13 \\
(-0.81)\end{array}$ \\
\hline \multicolumn{13}{|c|}{ Panel D: Stocks Sorted by RVC } \\
\hline & \multicolumn{6}{|c|}{ Equal-Weighted Returns and Alphas } & \multicolumn{6}{|c|}{ Value-Weighted Returns and Alphas } \\
\hline Quintile & 1 (Low) & 2 & 3 & 4 & 5 (High) & High-Low & 1 (Low) & 2 & 3 & 4 & 5 (High) & High-Low \\
\hline Mean Return & $\begin{array}{l}36.18 \\
(3.54)\end{array}$ & $\begin{array}{l}31.82 \\
(3.22)\end{array}$ & $\begin{array}{l}28.23 \\
(3.00)\end{array}$ & $\begin{array}{l}27.47 \\
(3.05)\end{array}$ & $\begin{array}{l}22.21 \\
(2.42)\end{array}$ & $\begin{array}{c}-13.97^{* *} \\
(-2.58)\end{array}$ & $\begin{array}{l}24.36 \\
(3.53)\end{array}$ & $\begin{array}{l}24.80 \\
(3.65)\end{array}$ & $\begin{array}{l}22.94 \\
(3.59)\end{array}$ & $\begin{array}{l}23.40 \\
(3.87)\end{array}$ & $\begin{array}{l}20.41 \\
(3.20)\end{array}$ & $\begin{array}{c}-3.95 \\
(-1.00)\end{array}$ \\
\hline Alpha & $\begin{array}{l}19.82 \\
(4.04)\end{array}$ & $\begin{array}{l}10.62 \\
(3.36)\end{array}$ & $\begin{array}{c}5.54 \\
(2.48)\end{array}$ & $\begin{array}{c}4.66 \\
(2.47)\end{array}$ & $\begin{array}{c}-0.94 \\
(-0.41)\end{array}$ & $\begin{array}{c}-20.76^{* * *} \\
(-3.87)\end{array}$ & $\begin{array}{c}4.27 \\
(1.76)\end{array}$ & $\begin{array}{c}3.29 \\
(1.62)\end{array}$ & $\begin{array}{c}2.42 \\
(1.30)\end{array}$ & $\begin{array}{l}3.27 \\
(2.10)\end{array}$ & $\begin{array}{c}0.06 \\
(0.07)\end{array}$ & $\begin{array}{c}-4.22 \\
(-1.46)\end{array}$ \\
\hline
\end{tabular}


Table 8. Double-Sorted Portfolios: Portfolios Sorted by Various Jump Variation Measures.

\begin{tabular}{|c|c|c|c|c|c|c|c|c|c|c|c|c|}
\hline \multicolumn{13}{|c|}{ Panel A: Stocks Sorted by SRVLJ, Controlling for SRVJ Based on $\gamma^{2}$} \\
\hline & \multicolumn{6}{|c|}{ Equal-Weighted Returns and Alphas } & \multicolumn{6}{|c|}{ Value-Weighted Returns and Alphas } \\
\hline & \multicolumn{6}{|c|}{ SRVJ Quintile } & \multicolumn{6}{|c|}{ SRVJ Quintile } \\
\hline SRVLJ Quintile & 1 (Low) & 2 & 3 & 4 & 5 (High) & Average & 1 (Low) & 2 & 3 & 4 & 5 (High) & Average \\
\hline \multicolumn{13}{|c|}{ Part I: Mean Return and Alpha } \\
\hline 1 (Low) & 47.99 & 28.73 & 21.12 & 12.90 & 7.82 & 23.71 & 41.88 & 18.83 & 19.24 & 13.59 & 5.04 & 16.61 \\
\hline 2 & 50.55 & 37.24 & 22.38 & 26.91 & 12.99 & 33.21 & 29.61 & 25.26 & 18.26 & 17.39 & 14.08 & 22.70 \\
\hline 3 & 53.07 & 38.57 & 23.49 & 21.73 & 12.37 & 29.75 & 36.31 & 26.28 & 8.10 & 15.50 & 17.40 & 24.19 \\
\hline 4 & 56.29 & 21.42 & 35.09 & 14.59 & 17.86 & 27.51 & 36.38 & 7.94 & 18.53 & 13.64 & 16.59 & 20.33 \\
\hline 5 (High) & 47.70 & 45.92 & 32.70 & 26.10 & 7.59 & 32.00 & 32.64 & 39.20 & 23.00 & 20.20 & 19.84 & 27.00 \\
\hline High-Low & -0.24 & 17.19 & 11.58 & 13.20 & -0.23 & 8.30 & 6.38 & 20.37 & 3.76 & 6.61 & 14.81 & 10.38 \\
\hline Alpha & -6.48 & 16.64 & 10.80 & 13.88 & 2.60 & 7.49 & 3.69 & 19.41 & 2.46 & 6.06 & 13.84 & 9.09 \\
\hline \multicolumn{13}{|l|}{ Part II: $t$-Statistics } \\
\hline 1 (Low) & 5.15 & 2.90 & 2.26 & 1.48 & 0.92 & 2.68 & 3.45 & 2.66 & 2.89 & 2.08 & 0.75 & 2.67 \\
\hline 2 & 4.81 & 3.62 & 1.94 & 0.81 & 1.43 & 3.49 & 3.97 & 3.40 & 2.28 & 0.88 & 1.99 & 3.49 \\
\hline 3 & 4.87 & 3.03 & 0.75 & 1.89 & 1.39 & 3.03 & 4.65 & 3.06 & 0.37 & 1.92 & 2.65 & 3.73 \\
\hline 4 & 4.64 & 0.63 & 3.07 & 1.53 & 2.04 & 2.81 & 4.26 & 0.27 & 2.18 & 2.05 & 2.50 & 3.04 \\
\hline 5 (High) & 4.39 & 4.29 & 3.23 & 2.71 & 0.89 & 3.38 & 3.77 & 4.89 & 3.16 & 2.78 & 2.99 & 4.01 \\
\hline High-Low & -0.04 & 4.24 & 3.31 & 3.87 & -0.06 & 4.18 & 1.09 & 3.71 & 0.79 & 1.54 & 3.09 & 4.15 \\
\hline Alpha & -1.19 & 4.11 & 3.06 & 4.09 & 0.67 & 3.80 & 0.62 & 3.40 & 0.49 & 1.44 & 2.96 & 3.53 \\
\hline \multicolumn{13}{|c|}{ Panel B: Stocks Sorted by SRVSJ, Controlling for SRVJ Based on $\gamma^{2}$} \\
\hline & \multicolumn{6}{|c|}{ Equal-Weighted Returns and Alphas } & \multicolumn{6}{|c|}{ Value-Weighted Returns and Alphas } \\
\hline & \multicolumn{6}{|c|}{ SRVJ Quintile } & \multicolumn{6}{|c|}{ SRVJ Quintile } \\
\hline SRVSJ Quintile & 1 (Low) & 2 & 3 & 4 & 5 (High) & Average & 1 (Low) & 2 & 3 & 4 & 5 (High) & Average \\
\hline \multicolumn{13}{|c|}{ Part I: Mean Return and Alpha } \\
\hline 1 (Low) & 56.90 & 44.50 & 34.78 & 26.13 & 19.50 & 36.36 & 41.88 & 35.91 & 26.98 & 18.28 & 22.43 & 29.73 \\
\hline 2 & 55.66 & 47.02 & 30.91 & 18.59 & 10.34 & 32.50 & 40.76 & 34.11 & 20.84 & 13.23 & 16.52 & 25.09 \\
\hline 3 & 56.24 & 41.20 & 28.46 & 19.74 & 10.02 & 31.13 & 30.72 & 23.04 & 20.39 & 13.82 & 14.29 & 20.45 \\
\hline 4 & 53.58 & 37.21 & 16.75 & 12.62 & 10.19 & 26.07 & 28.23 & 23.82 & 12.02 & 14.94 & 6.93 & 17.19 \\
\hline 5 (High) & 34.30 & 25.12 & 19.72 & 12.12 & 5.79 & 19.41 & 17.03 & 16.65 & 18.03 & 12.69 & 3.77 & 13.63 \\
\hline High-Low & -22.60 & -19.38 & -15.06 & -14.01 & -13.71 & -16.95 & -28.00 & -19.26 & -8.95 & -5.59 & -18.66 & -16.09 \\
\hline Alpha & -19.26 & -18.83 & -14.19 & -14.86 & -16.20 & -16.67 & -25.86 & -20.41 & -6.87 & -4.79 & -18.22 & -15.23 \\
\hline
\end{tabular}


Table 8. Cont.

\begin{tabular}{|c|c|c|c|c|c|c|c|c|c|c|c|c|}
\hline \multicolumn{13}{|c|}{ Panel B: Stocks Sorted by SRVSJ, Controlling for SRVJ Based on $\gamma^{2}$} \\
\hline & \multicolumn{6}{|c|}{ Equal-Weighted Returns and Alphas } & \multicolumn{6}{|c|}{ Value-Weighted Returns and Alphas } \\
\hline & \multicolumn{6}{|c|}{ SRVJ Quintile } & \multicolumn{6}{|c|}{ SRVJ Quintile } \\
\hline SRVSJ Quintile & 1 (Low) & 2 & 3 & 4 & 5 (High) & Average & 1 (Low) & 2 & 3 & 4 & 5 (High) & Average \\
\hline \multicolumn{13}{|l|}{ Part II: $t$-Statistics } \\
\hline 1 (Low) & 5.44 & 4.22 & 3.40 & 2.67 & 2.16 & 3.76 & 5.47 & 4.75 & 3.58 & 2.54 & 3.33 & 4.44 \\
\hline 2 & 5.20 & 4.21 & 3.08 & 1.93 & 1.17 & 3.35 & 5.19 & 4.65 & 3.00 & 1.95 & 2.33 & 3.86 \\
\hline 3 & 5.15 & 3.99 & 2.77 & 2.14 & 1.17 & 3.26 & 4.04 & 3.21 & 2.89 & 2.02 & 2.20 & 3.20 \\
\hline 4 & 5.33 & 3.58 & 1.71 & 1.42 & 1.23 & 2.86 & 3.39 & 3.41 & 1.72 & 2.22 & 1.02 & 2.68 \\
\hline 5 (High) & 3.46 & 2.60 & 2.12 & 1.39 & 0.70 & 2.19 & 2.22 & 2.32 & 2.67 & 1.89 & 0.56 & 2.16 \\
\hline High-Low & -4.99 & -5.04 & -4.14 & -3.79 & -3.45 & -7.22 & -5.37 & -3.66 & -1.79 & -1.19 & -3.80 & -5.77 \\
\hline Alpha & -4.15 & -4.90 & -3.91 & -4.16 & -4.12 & -7.42 & -4.93 & -3.65 & -1.30 & -1.02 & -3.78 & -5.32 \\
\hline \multicolumn{13}{|c|}{ Panel C: Stocks Sorted by SRVSJ, Controlling for SRVLJ Based on $\gamma^{2}$} \\
\hline & \multicolumn{6}{|c|}{ Equal-Weighted Returns and Alphas } & \multicolumn{6}{|c|}{ Value-Weighted Returns and Alphas } \\
\hline & \multicolumn{6}{|c|}{ SRVLJ Quintile } & \multicolumn{6}{|c|}{ SRVLJ Quintile } \\
\hline SRVSJ Quintile & 1 (Low) & 2 & 3 & 4 & 5 (High) & Average & 1 (Low) & 2 & 3 & 4 & 5 (High) & Average \\
\hline \multicolumn{13}{|c|}{ Part I: Mean Return and Alpha } \\
\hline 1 (Low) & 60.34 & 51.38 & 40.69 & 44.93 & 31.91 & 47.28 & 41.88 & 35.33 & 35.90 & 34.10 & 30.94 & 36.43 \\
\hline 2 & 57.30 & 38.41 & 58.52 & 28.26 & 28.35 & 40.27 & 23.32 & 25.07 & 37.49 & 18.00 & 25.93 & 25.12 \\
\hline 3 & 35.25 & 26.42 & 4.21 & 28.76 & 14.62 & 25.17 & 16.19 & 21.74 & 9.63 & 20.36 & 21.71 & 19.86 \\
\hline 4 & 28.42 & 16.02 & 48.13 & 10.74 & 10.78 & 19.49 & 17.78 & 15.39 & 97.02 & 14.92 & 14.12 & 22.20 \\
\hline 5 (High) & 18.79 & 9.45 & -1.20 & 8.01 & 8.26 & 10.89 & 14.98 & 8.09 & 6.98 & 7.78 & 13.31 & 11.30 \\
\hline High-Low & -41.55 & -41.93 & -40.72 & -38.27 & -23.65 & -36.71 & -26.63 & -27.24 & -27.87 & -27.67 & -17.63 & -25.38 \\
\hline Alpha & -40.15 & -41.33 & -40.17 & -37.25 & -22.90 & -35.45 & -25.59 & -25.58 & -26.01 & -26.35 & -17.73 & -24.07 \\
\hline \multicolumn{13}{|l|}{ Part II: $t$-Statistics } \\
\hline 1 (Low) & 5.60 & 4.76 & 1.56 & 4.18 & 3.28 & 4.58 & 5.38 & 4.76 & 1.72 & 4.12 & 4.37 & 5.13 \\
\hline 2 & 5.40 & 3.61 & 2.17 & 2.64 & 3.02 & 3.97 & 3.03 & 3.41 & 1.61 & 2.34 & 3.60 & 3.62 \\
\hline 3 & 3.58 & 2.60 & 0.19 & 2.85 & 1.62 & 2.65 & 2.27 & 3.21 & 0.53 & 2.93 & 3.11 & 3.15 \\
\hline 4 & 2.83 & 1.70 & 1.20 & 1.10 & 1.23 & 2.01 & 2.49 & 2.30 & 1.11 & 2.03 & 2.07 & 2.35 \\
\hline 5 (High) & 2.12 & 1.06 & -0.06 & 0.93 & 1.01 & 1.31 & 2.12 & 1.22 & 0.46 & 1.08 & 2.02 & 1.82 \\
\hline High-Low & -8.29 & -8.49 & -2.04 & -6.08 & -5.33 & -8.59 & -5.15 & -5.54 & -1.50 & -4.14 & -3.64 & -6.61 \\
\hline Alpha & -8.24 & -8.75 & -2.03 & -6.22 & -5.50 & -8.80 & -4.90 & -5.18 & -1.39 & -3.87 & -3.66 & -6.13 \\
\hline
\end{tabular}


Table 8. Cont.

\begin{tabular}{|c|c|c|c|c|c|c|c|c|c|c|c|c|}
\hline \multicolumn{13}{|c|}{ Panel D: Stocks Sorted by SRVLJ, Controlling for SRVSJ Based on $\gamma^{2}$} \\
\hline & \multicolumn{7}{|c|}{ Equal-Weighted Returns and Alphas } & \multicolumn{5}{|c|}{ Value-Weighted Returns and Alphas } \\
\hline & \multicolumn{6}{|c|}{ SRVSJ Quintile } & \multicolumn{6}{|c|}{ SRVSJ Quintile } \\
\hline SRVLJ Quintile & 1 (Low) & 2 & 3 & 4 & 5 (High) & Average & 1 (Low) & 2 & 3 & 4 & 5 (High) & Average \\
\hline \multicolumn{13}{|c|}{ Part I: Mean Return and Alpha } \\
\hline 1 (Low) & 59.99 & 60.01 & 41.84 & 27.66 & 16.62 & 41.23 & 41.88 & 30.53 & 19.67 & 16.32 & 12.01 & 23.80 \\
\hline 2 & 49.75 & 41.95 & 27.20 & 17.65 & 11.51 & 29.31 & 35.69 & 25.91 & 18.85 & 17.68 & 8.92 & 20.89 \\
\hline 3 & 76.41 & 38.05 & 24.45 & 6.48 & 14.41 & 32.06 & 45.15 & 16.78 & 11.30 & 10.47 & 21.45 & 21.03 \\
\hline 4 & 43.99 & 30.88 & 23.29 & 13.82 & 11.84 & 24.86 & 38.54 & 18.80 & 17.59 & 15.79 & 10.15 & 20.55 \\
\hline 5 (High) & 32.38 & 28.64 & 10.59 & 8.97 & 6.78 & 17.47 & 31.35 & 25.93 & 18.89 & 13.97 & 10.03 & 20.03 \\
\hline High-Low & -27.61 & -31.38 & -31.25 & -18.69 & -9.84 & -23.75 & -9.14 & -4.60 & -0.78 & -2.35 & -1.98 & -3.77 \\
\hline Alpha & -26.51 & -31.63 & -31.28 & -19.37 & -9.80 & -23.72 & -8.62 & -3.72 & -0.59 & -2.79 & -1.66 & -3.47 \\
\hline \multicolumn{13}{|l|}{ Part II: $t$-Statistics } \\
\hline 1 (Low) & 5.51 & 5.59 & 4.22 & 2.85 & 1.85 & 4.25 & 5.37 & 4.03 & 2.69 & 2.23 & 1.71 & 3.59 \\
\hline 2 & 4.51 & 3.85 & 2.62 & 1.87 & 1.29 & 3.05 & 4.71 & 3.54 & 2.63 & 2.71 & 1.32 & 3.28 \\
\hline 3 & 2.31 & 1.95 & 1.61 & 0.41 & 0.80 & 2.49 & 1.53 & 1.16 & 1.08 & 0.78 & 1.49 & 2.25 \\
\hline 4 & 4.05 & 2.90 & 2.28 & 1.44 & 1.31 & 2.63 & 4.83 & 2.51 & 2.47 & 2.31 & 1.39 & 3.23 \\
\hline 5 (High) & 3.36 & 2.99 & 1.21 & 1.03 & 0.83 & 2.02 & 4.32 & 3.63 & 2.69 & 2.11 & 1.55 & 3.23 \\
\hline High-Low & -6.48 & -7.66 & -6.92 & -4.65 & -2.73 & -8.80 & -1.83 & -0.98 & -0.16 & -0.46 & -0.44 & -1.49 \\
\hline Alpha & -6.16 & -7.50 & -6.94 & -4.94 & -2.83 & -8.94 & -1.70 & -0.79 & -0.12 & -0.53 & -0.37 & -1.38 \\
\hline
\end{tabular}

Notes: See notes to Table 5. This table presents average returns (called "Mean Return") and risk-adjusted alphas (called "Alpha") for portfolios sorted by various jump variation measures. The sample includes NYSE, NASDAQ and AMEX listed stocks for the period January 1993 to December 2016. At the end of each Tuesday, all the stocks in the sample are sorted into quintile portfolios based on ascending values of SRVJ (SRVLJ/SRVSJ). Then, within each quintile portfolio, stocks are further sorted based on the values of SRVLJ/SRVSJ (SRVSJ/SRVLJ), resulting in 25 portfolios. Each portfolio is held for one week. The row labeled "High-Low" reports the average values of one-week-ahead returns in Part I (corresponding Newey-West $t$-statistics are given in Part II of the panel). The row labeled "Alpha" reports Fama-French-Carhart four-factor alphas in Part I (corresponding Newey-West $t$-statistics are again given in Part II of the panel) for the double-sorted High-Low portfolios. Please note that entries given in the "Average" column of the table, are average returns across the 5 quintiles. Finally, note that SRVLJ and SRVSJ are constructed based on jump truncation level $\gamma^{2}=5 \sqrt{\frac{1}{t} \widehat{I V}_{t}^{(i)}} \Delta_{n}^{0.49}$. 
Table 9. Double-Sorted Portfolios: Portfolios Sorted by SRVJ and RSK.

Panel A: Stocks Sorted by SRVJ, Controlling for RSK

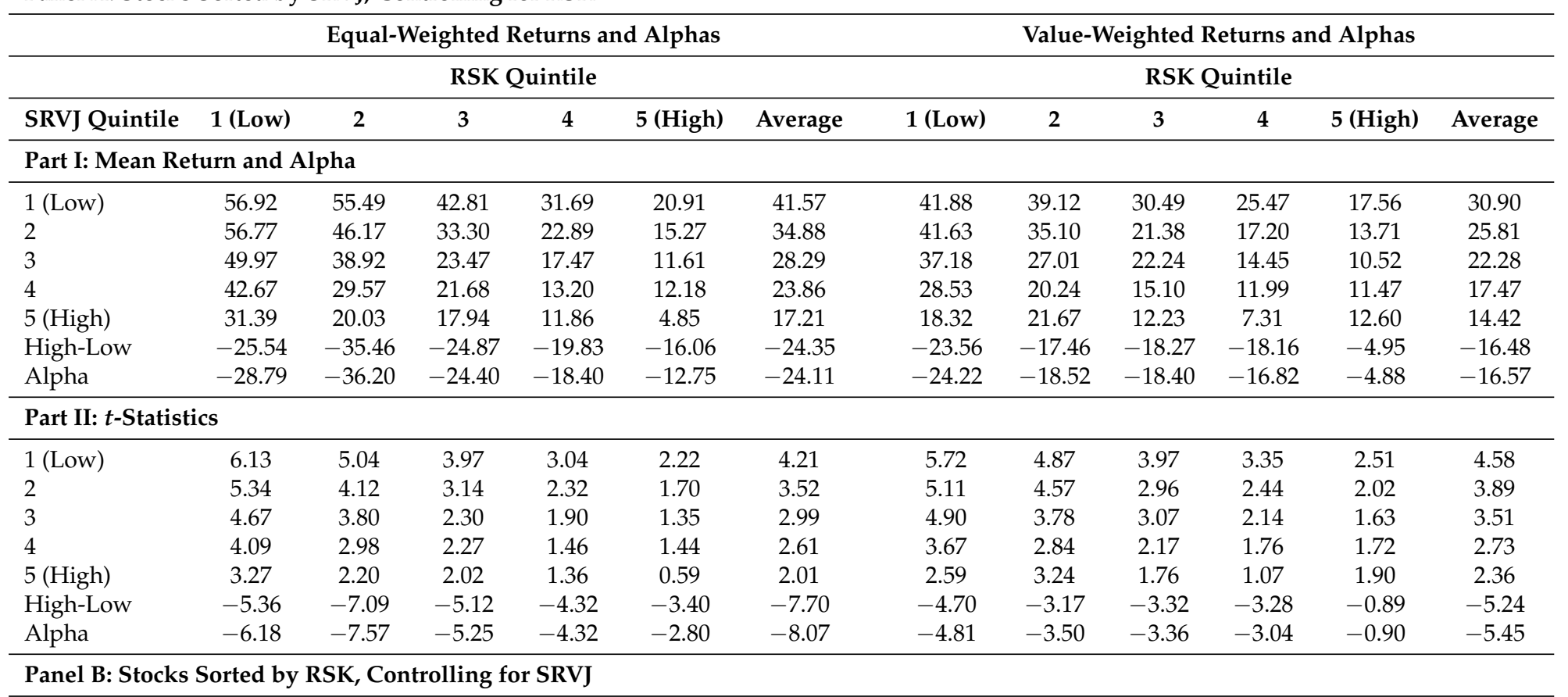

Equal-Weighted Returns and Alphas

Value-Weighted Returns and Alphas

\begin{tabular}{|c|c|c|c|c|c|c|c|c|c|c|c|c|}
\hline & \multicolumn{6}{|c|}{ Equal-Weignted Returns and AIphas } & \multicolumn{6}{|c|}{ Value-Weighted Returns and Alphas } \\
\hline \multicolumn{13}{|c|}{ Part I: Mean Return and Alpha } \\
\hline 1 (Low) & 51.14 & 29.34 & 21.71 & 17.58 & 12.40 & 26.43 & 41.88 & 18.33 & 20.91 & 12.07 & 4.09 & 18.48 \\
\hline 2 & 49.50 & 39.74 & 23.05 & 14.15 & 11.46 & 27.58 & 37.29 & 33.29 & 14.95 & 10.36 & 10.00 & 21.18 \\
\hline 3 & 49.96 & 37.41 & 27.26 & 18.14 & 12.93 & 29.14 & 28.38 & 23.82 & 22.52 & 14.80 & 15.23 & 20.95 \\
\hline 4 & 53.85 & 43.31 & 28.11 & 18.79 & 12.52 & 31.32 & 36.37 & 28.73 & 19.57 & 17.15 & 10.67 & 22.50 \\
\hline Alpha & 0.54 & 16.64 & 8.57 & 2.34 & -4.54 & 4.71 & -0.71 & 15.66 & 4.39 & 6.30 & 9.15 & 6.96 \\
\hline
\end{tabular}


Table 9. Cont.

\begin{tabular}{|c|c|c|c|c|c|c|c|c|c|c|c|c|}
\hline \multicolumn{13}{|c|}{ Panel B: Stocks Sorted by RSK, Controlling for SRVJ } \\
\hline & \multicolumn{6}{|c|}{ Equal-Weighted Returns and Alphas } & \multicolumn{6}{|c|}{ Value-Weighted Returns and Alphas } \\
\hline & \multicolumn{6}{|c|}{ SRVJ Quintile } & \multicolumn{6}{|c|}{ SRVJ Quintile } \\
\hline RSK Quintile & 1 (Low) & 2 & 3 & 4 & 5 (High) & Average & 1 (Low) & 2 & 3 & 4 & 5 (High) & Average \\
\hline \multicolumn{13}{|c|}{ Part II: $t$-Statistics } \\
\hline 1 (Low) & 5.56 & 3.07 & 2.36 & 1.99 & 1.42 & 3.02 & 4.98 & 2.61 & 3.15 & 1.74 & 0.60 & 2.97 \\
\hline 2 & 4.76 & 3.75 & 2.33 & 1.57 & 1.30 & 2.94 & 4.89 & 4.50 & 2.06 & 1.53 & 1.52 & 3.29 \\
\hline 3 & 4.76 & 3.60 & 2.69 & 1.94 & 1.50 & 3.07 & 3.59 & 3.33 & 3.29 & 2.24 & 2.33 & 3.30 \\
\hline 4 & 4.99 & 3.98 & 2.77 & 1.97 & 1.49 & 3.27 & 4.70 & 3.89 & 2.69 & 2.39 & 1.63 & 3.45 \\
\hline 5 (High) & 5.00 & 4.19 & 2.99 & 2.17 & 0.68 & 3.27 & 4.50 & 4.20 & 3.29 & 2.83 & 2.09 & 3.87 \\
\hline High-Low & 0.85 & 3.98 & 2.31 & 0.84 & -1.61 & 2.35 & -0.05 & 2.85 & 0.74 & 1.48 & 2.19 & 2.87 \\
\hline Alpha & 0.14 & 4.40 & 2.24 & 0.66 & -1.09 & 2.42 & -0.14 & 3.05 & 0.89 & 1.38 & 2.01 & 2.92 \\
\hline
\end{tabular}

Notes: See notes to Table 5. This table presents average returns (called "Mean Return") and risk-adjusted alphas (called "Alpha") for portfolios sorted by SRVJ controlling for RSK, and vice versa. The sample includes NYSE, NASDAQ and AMEX listed stocks for the period January 1993 to December 2016. At the end of each Tuesday, all the stocks in the sample are sorted into quintile portfolios based on ascending values of RSK (SRVI), and then within each quintile portfolio, stocks are further sorted using values of SRVI (RSK), resulting in 25 portfolios. Each portfolio is held for one week. The row labeled "High-Low" reports the average values of one-week-ahead returns in Part I (corresponding Newey-West $t$-statistics are given in Part II of the panel). The row labeled "Alpha" reports Fama-French-Carhart four-factor alphas in Part I (corresponding Newey-West $t$-statistics are again given in Part II of the panel) for each of the quintile portfolios, as well as for the average across 5 RSK (SRVJ) portfolios.

Table 10. Double-Sorted Portfolios: Portfolios Sorted by SRVLJ/SRVSJ, Controlling for RSK.

\begin{tabular}{|c|c|c|c|c|c|c|c|c|c|c|c|c|}
\hline \multicolumn{13}{|c|}{ Panel A: Stocks Sorted by SRVLJ, Controlling for RSK Based on $\gamma^{2}$} \\
\hline & \multicolumn{6}{|c|}{ Equal-Weighted Returns and Alphas } & \multicolumn{6}{|c|}{ Value-Weighted Returns and Alphas } \\
\hline & \multicolumn{6}{|c|}{ RSK Quintile } & \multicolumn{6}{|c|}{ RSK Quintile } \\
\hline SRVLJ Quintile & 1 (Low) & 2 & 3 & 4 & 5 (High) & Average & 1 (Low) & 2 & 3 & 4 & 5 (High) & Average \\
\hline \multicolumn{13}{|c|}{ Part I: Mean Return and Alpha } \\
\hline 1 (Low) & 47.31 & 31.04 & 20.83 & 13.46 & 9.23 & 24.38 & 41.88 & 20.74 & 17.63 & 10.28 & 10.95 & 17.04 \\
\hline 2 & 48.24 & 35.53 & 18.15 & 41.82 & 10.40 & 30.72 & 27.52 & 25.93 & 13.06 & 22.40 & 13.21 & 21.88 \\
\hline 3 & 46.79 & 36.39 & -2.22 & 17.53 & 16.01 & 27.86 & 27.77 & 24.25 & 23.68 & 13.64 & 18.71 & 21.29 \\
\hline 4 & 47.56 & 9.61 & 27.36 & 19.04 & 20.92 & 27.46 & 27.71 & 8.29 & 20.27 & 17.45 & 16.06 & 20.34 \\
\hline 5 (High) & 43.55 & 43.48 & 34.14 & 26.41 & 7.84 & 31.13 & 35.80 & 32.07 & 20.61 & 23.70 & 18.89 & 26.28 \\
\hline High-Low & -3.57 & 12.44 & 13.30 & 12.95 & -1.39 & 6.75 & 10.42 & 11.33 & 2.98 & 13.42 & 7.94 & 9.22 \\
\hline Alpha & -8.76 & 11.52 & 13.22 & 13.09 & 1.59 & 6.13 & 8.26 & 11.20 & 1.91 & 12.69 & 7.30 & 8.27 \\
\hline
\end{tabular}


Table 10. Cont.

\begin{tabular}{|c|c|c|c|c|c|c|c|c|c|c|c|c|}
\hline \multicolumn{13}{|c|}{ Panel A: Stocks Sorted by SRVLJ, Controlling for RSK Based on $\gamma^{2}$} \\
\hline & \multicolumn{6}{|c|}{ Equal-Weighted Returns and Alphas } & \multicolumn{6}{|c|}{ Value-Weighted Returns and Alphas } \\
\hline & \multicolumn{6}{|c|}{ RSK Quintile } & \multicolumn{6}{|c|}{ RSK Quintile } \\
\hline SRVLJ Quintile & 1 (Low) & 2 & 3 & 4 & 5 (High) & Average & 1 (Low) & 2 & 3 & 4 & 5 (High) & Average \\
\hline \multicolumn{13}{|l|}{ Part II: $t$-Statistics } \\
\hline 1 (Low) & 5.10 & 3.13 & 2.17 & 1.51 & 1.08 & 2.73 & 3.34 & 2.85 & 2.51 & 1.59 & 1.63 & 2.68 \\
\hline 2 & 4.67 & 3.47 & 1.41 & 1.61 & 1.16 & 3.21 & 3.71 & 3.71 & 1.47 & 1.12 & 1.98 & 3.42 \\
\hline 3 & 4.48 & 2.95 & -0.07 & 1.54 & 1.79 & 2.91 & 3.81 & 2.87 & 0.85 & 1.56 & 2.89 & 3.30 \\
\hline 4 & 4.32 & 0.37 & 2.27 & 1.96 & 2.38 & 2.89 & 3.68 & 0.43 & 2.12 & 2.52 & 2.45 & 3.17 \\
\hline 5 (High) & 4.22 & 4.03 & 3.42 & 2.75 & 0.93 & 3.34 & 4.08 & 3.80 & 2.81 & 3.23 & 2.79 & 3.90 \\
\hline High-Low & -0.66 & 2.93 & 4.28 & 4.11 & -0.37 & 3.64 & 1.71 & 1.89 & 0.66 & 3.03 & 1.62 & 3.76 \\
\hline Alpha & -1.62 & 2.63 & 4.14 & 4.13 & 0.42 & 3.18 & 1.35 & 1.76 & 0.41 & 2.90 & 1.51 & 3.22 \\
\hline \multicolumn{13}{|c|}{ Panel B: Stocks Sorted by SRVSJ, Controlling for RSK Based on $\gamma^{2}$} \\
\hline & \multicolumn{6}{|c|}{ Equal-Weighted Returns and Alphas } & \multicolumn{6}{|c|}{ Value-Weighted Returns and Alphas } \\
\hline & \multicolumn{6}{|c|}{ RSK Quintile } & \multicolumn{6}{|c|}{ RSK Quintile } \\
\hline SRVSJ Quintile & 1 (Low) & 2 & 3 & 4 & 5 (High) & Average & 1 (Low) & 2 & 3 & 4 & 5 (High) & Average \\
\hline \multicolumn{13}{|c|}{ Part I: Mean Return and Alpha } \\
\hline 1 (Low) & 56.15 & 51.19 & 42.56 & 31.93 & 24.16 & 41.20 & 41.88 & 40.69 & 31.16 & 31.49 & 21.39 & 33.55 \\
\hline 2 & 54.34 & 50.15 & 35.79 & 24.76 & 12.89 & 35.59 & 32.45 & 33.20 & 21.93 & 19.73 & 23.95 & 26.25 \\
\hline 3 & 55.24 & 38.53 & 26.29 & 18.62 & 11.09 & 29.95 & 27.91 & 22.64 & 18.61 & 12.36 & 16.37 & 19.58 \\
\hline 4 & 40.20 & 31.45 & 18.77 & 14.26 & 12.14 & 23.35 & 18.13 & 22.01 & 15.30 & 13.17 & 10.40 & 15.79 \\
\hline 5 (High) & 30.21 & 18.90 & 15.72 & 7.49 & 4.76 & 15.42 & 18.59 & 19.31 & 12.59 & 8.23 & 6.56 & 13.06 \\
\hline High-Low & -25.94 & -32.29 & -26.84 & -24.43 & -19.40 & -25.78 & -24.44 & -21.37 & -18.56 & -23.26 & -14.84 & -20.49 \\
\hline Alpha & -23.86 & -31.76 & -26.49 & -23.94 & -20.71 & -25.35 & -22.27 & -22.89 & -18.30 & -21.83 & -14.44 & -19.95 \\
\hline \multicolumn{13}{|c|}{ Part II: $t$-Statistics } \\
\hline 1 (Low) & 5.43 & 4.75 & 3.98 & 3.16 & 2.62 & 4.15 & 5.11 & 5.25 & 4.16 & 4.39 & 3.15 & 4.92 \\
\hline 2 & 5.17 & 4.48 & 3.42 & 2.47 & 1.43 & 3.61 & 4.30 & 4.53 & 2.96 & 2.68 & 3.39 & 3.95 \\
\hline 3 & 5.25 & 3.76 & 2.60 & 1.96 & 1.25 & 3.14 & 3.75 & 3.12 & 2.66 & 1.72 & 2.46 & 3.03 \\
\hline 4 & 4.11 & 3.13 & 1.96 & 1.58 & 1.45 & 2.59 & 2.50 & 3.20 & 2.27 & 1.98 & 1.57 & 2.55 \\
\hline 5 (High) & 3.18 & 2.06 & 1.71 & 0.87 & 0.58 & 1.79 & 2.56 & 2.86 & 1.79 & 1.23 & 0.97 & 2.09 \\
\hline High-Low & -5.78 & -7.15 & -6.04 & -5.58 & -4.72 & -8.41 & -4.21 & -4.04 & -3.67 & -4.56 & -2.93 & -6.31 \\
\hline Alpha & -5.36 & -7.20 & -6.12 & -5.75 & -5.27 & -8.89 & -3.83 & -4.29 & -3.51 & -4.09 & -2.83 & -6.01 \\
\hline
\end{tabular}

Notes: See notes to Table 8. Portfolios are sorted by SRVLJ/SRVSJ, controlling for RSK, and using truncation level $\gamma^{2}$, as discussed in the notes to Table 2. 
Table 11. Double-Sorted Portfolios: Portfolios Sorted by RSK, Controlling for SRVLJ or SRVSJ.

\begin{tabular}{|c|c|c|c|c|c|c|c|c|c|c|c|c|}
\hline \multicolumn{13}{|c|}{ Panel A: Stocks Sorted by RSK, Controlling for SRVLJ Based on $\gamma^{2}$} \\
\hline & \multicolumn{6}{|c|}{ Equal-Weighted Returns and Alphas } & \multicolumn{6}{|c|}{ Value-Weighted Returns and Alphas } \\
\hline & \multicolumn{6}{|c|}{ SRVLJ Quintile } & \multicolumn{6}{|c|}{ SRVLJ Quintile } \\
\hline RSK Quintile & 1 (Low) & 2 & 3 & 4 & 5 (High) & Average & 1 (Low) & 2 & 3 & 4 & 5 (High) & Average \\
\hline \multicolumn{13}{|c|}{ Part I: Mean Return and Alpha } \\
\hline 1 (Low) & 51.98 & 46.26 & 32.88 & 42.99 & 35.40 & 43.72 & 41.88 & 34.45 & 32.97 & 31.19 & 33.46 & 34.64 \\
\hline 2 & 48.38 & 38.05 & 55.49 & 31.86 & 20.58 & 37.03 & 30.03 & 23.16 & 48.38 & 17.47 & 16.06 & 24.56 \\
\hline 3 & 43.16 & 26.83 & 8.78 & 20.69 & 16.54 & 25.94 & 25.91 & 21.10 & 10.09 & 19.92 & 19.16 & 21.15 \\
\hline 4 & 37.70 & 22.51 & 55.61 & 11.20 & 14.84 & 24.67 & 18.23 & 18.69 & 8.48 & 13.82 & 14.50 & 16.23 \\
\hline 5 (High) & 21.43 & 8.10 & -0.87 & 14.29 & 7.74 & 12.81 & 13.59 & 7.52 & -8.20 & 15.74 & 15.50 & 12.31 \\
\hline High-Low & -30.55 & -38.16 & -30.25 & -30.06 & -27.66 & -31.07 & -23.12 & -26.93 & -37.56 & -16.82 & -17.96 & -22.43 \\
\hline Alpha & -32.89 & -37.85 & -28.79 & -30.01 & -24.81 & -30.46 & -23.34 & -24.74 & -36.67 & -14.80 & -18.66 & -21.39 \\
\hline \multicolumn{13}{|c|}{ Part II: $t$-Statistics } \\
\hline 1 (Low) & 5.63 & 4.46 & 1.33 & 4.16 & 3.61 & 4.48 & 4.94 & 4.65 & 1.67 & 4.00 & 4.80 & 5.06 \\
\hline 2 & 4.68 & 3.68 & 2.16 & 3.04 & 2.22 & 3.72 & 3.99 & 3.28 & 2.04 & 2.34 & 2.31 & 3.63 \\
\hline 3 & 4.14 & 2.66 & 0.40 & 2.04 & 1.84 & 2.69 & 3.32 & 3.22 & 0.59 & 2.66 & 2.87 & 3.29 \\
\hline 4 & 3.62 & 2.29 & 1.37 & 1.11 & 1.73 & 2.50 & 2.54 & 2.82 & 0.48 & 1.80 & 2.22 & 2.54 \\
\hline 5 (High) & 2.27 & 0.89 & -0.04 & 1.63 & 0.92 & 1.49 & 1.91 & 1.08 & -0.52 & 2.33 & 2.24 & 1.98 \\
\hline High-Low & -7.91 & -9.22 & -1.71 & -5.63 & -5.85 & -9.46 & -4.56 & -6.02 & -2.29 & -2.75 & -3.47 & -6.54 \\
\hline Alpha & -8.43 & -9.50 & -1.64 & -5.86 & -5.51 & -9.57 & -4.59 & -5.54 & -2.22 & -2.37 & -3.65 & -6.10 \\
\hline \multicolumn{13}{|c|}{ Panel B: Stocks Sorted by RSK, Controlling for SRVSJ Based on $\gamma^{2}$} \\
\hline & \multicolumn{6}{|c|}{ Equal-Weighted Returns and Alphas } & \multicolumn{6}{|c|}{ Value-Weighted Returns and Alphas } \\
\hline & \multicolumn{6}{|c|}{ SRVSJ Quintile } & \multicolumn{6}{|c|}{ SRVSJ Quintile } \\
\hline RSK Quintile & 1 (Low) & 2 & 3 & 4 & 5 (High) & Average & 1 (Low) & 2 & 3 & 4 & 5 (High) & Average \\
\hline \multicolumn{13}{|c|}{ Part I: Mean Return and Alpha } \\
\hline 1 (Low) & 57.49 & 56.03 & 40.07 & 26.79 & 17.75 & 39.62 & 41.88 & 24.28 & 18.84 & 16.57 & 12.21 & 23.20 \\
\hline 2 & 51.21 & 42.88 & 31.02 & 15.27 & 12.69 & 30.61 & 35.14 & 26.67 & 14.77 & 21.10 & 9.49 & 21.43 \\
\hline 3 & 54.96 & 41.34 & 25.81 & 20.97 & 10.12 & 30.64 & 37.20 & 24.66 & 20.50 & 10.79 & 7.58 & 20.15 \\
\hline 4 & 42.52 & 38.93 & 29.50 & 16.21 & 9.58 & 27.35 & 37.22 & 23.08 & 17.57 & 15.29 & 11.02 & 20.84 \\
\hline 5 (High) & 33.23 & 28.85 & 9.62 & 10.20 & 5.82 & 17.54 & 31.94 & 30.24 & 21.43 & 16.36 & 8.89 & 21.77 \\
\hline High-Low & -24.26 & -27.17 & -30.45 & -16.59 & -11.93 & -22.08 & -12.15 & 5.96 & 2.59 & -0.21 & -3.33 & -1.43 \\
\hline Alpha & -22.78 & -27.83 & -30.17 & -17.16 & -11.85 & -21.96 & -10.86 & 7.74 & 2.94 & -0.96 & -3.03 & -0.83 \\
\hline
\end{tabular}


Table 11. Cont.

\begin{tabular}{|c|c|c|c|c|c|c|c|c|c|c|c|c|}
\hline \multicolumn{13}{|c|}{ Panel B: Stocks Sorted by RSK, Controlling for SRVSJ Based on $\gamma^{2}$} \\
\hline & \multicolumn{6}{|c|}{ Equal-Weighted Returns and Alphas } & \multicolumn{6}{|c|}{ Value-Weighted Returns and Alphas } \\
\hline & \multicolumn{6}{|c|}{ SRVSJ Quintile } & \multicolumn{6}{|c|}{ SRVSJ Quintile } \\
\hline RSK Quintile & 1 (Low) & 2 & 3 & 4 & 5 (High) & Average & 1 (Low) & 2 & 3 & 4 & 5 (High) & Average \\
\hline \multicolumn{13}{|c|}{ Part II: $t$-Statistics } \\
\hline 1 (Low) & 5.53 & 5.32 & 4.11 & 2.82 & 1.99 & 4.19 & 5.79 & 3.21 & 2.52 & 2.21 & 1.73 & 3.49 \\
\hline 2 & 4.84 & 4.00 & 3.06 & 1.62 & 1.44 & 3.20 & 4.45 & 3.56 & 2.02 & 3.25 & 1.42 & 3.30 \\
\hline 3 & 4.96 & 3.79 & 2.58 & 2.21 & 1.15 & 3.15 & 4.86 & 3.35 & 3.01 & 1.55 & 1.13 & 3.12 \\
\hline 4 & 4.01 & 3.79 & 2.86 & 1.73 & 1.12 & 2.87 & 5.03 & 3.26 & 2.44 & 2.17 & 1.62 & 3.22 \\
\hline 5 (High) & 3.41 & 3.05 & 1.09 & 1.17 & 0.72 & 2.03 & 4.19 & 4.35 & 2.91 & 2.49 & 1.38 & 3.48 \\
\hline High-Low & -5.79 & -6.79 & -6.74 & -4.40 & -3.12 & -8.56 & -2.27 & 1.17 & 0.48 & -0.04 & -0.69 & -0.49 \\
\hline Alpha & -5.33 & -6.78 & -6.68 & -4.61 & -3.19 & -8.57 & -2.06 & 1.50 & 0.53 & -0.17 & -0.61 & -0.29 \\
\hline
\end{tabular}

Notes: See notes to Table 8. Portfolios are sorted by RSK, controlling for SRVLJ/SRVSJ, and using truncation level $\gamma^{2}$, as discussed in the notes to Table 2.

Table 12. Double-Sorted Portfolios: Portfolios Independently Sorted by Stock- and Industry-Level SRVJ.

\begin{tabular}{|c|c|c|c|c|c|c|c|c|c|c|c|c|c|c|}
\hline \multirow[b]{3}{*}{ Stock-Level Quintile } & \multicolumn{6}{|c|}{ Equal-Weighted Returns and Alphas } & \multicolumn{8}{|c|}{ Value-Weighted Returns and Alphas } \\
\hline & \multicolumn{6}{|c|}{ Industry-Level Quintile } & \multicolumn{8}{|c|}{ Industry-Level Quintile } \\
\hline & 1 (Low) & 2 & 3 & 4 & 5 (High) & High-Low & Alpha & 1 (Low) & 2 & 3 & 4 & 5 (High) & High-Low & Alpha \\
\hline \multicolumn{15}{|c|}{ Part I: Mean Return and Alpha } \\
\hline 1 (Low) & 39.23 & 47.42 & 53.00 & 61.43 & 72.18 & 32.94 & 34.74 & 36.50 & 33.40 & 32.78 & 36.58 & 25.03 & 10.19 & 9.28 \\
\hline 2 & 30.55 & 33.28 & 38.69 & 48.44 & 55.77 & 25.22 & 25.86 & 28.37 & 27.31 & 20.24 & 37.10 & 14.64 & 7.20 & 6.68 \\
\hline 3 & 14.07 & 21.47 & 24.27 & 30.98 & 43.15 & 29.07 & 29.60 & 18.16 & 24.85 & 17.56 & 20.35 & 8.69 & 10.64 & 11.98 \\
\hline 4 & 6.90 & 12.45 & 15.65 & 19.51 & 34.51 & 27.61 & 28.30 & 12.97 & 11.96 & 10.38 & 14.58 & 2.67 & 9.54 & 9.99 \\
\hline 5 (High) & -7.35 & 1.99 & 9.90 & 16.40 & 25.94 & 33.29 & 32.92 & -4.30 & 9.86 & 4.86 & 13.25 & -3.95 & 19.82 & 21.72 \\
\hline Alpha & -44.64 & -45.23 & -41.18 & -45.96 & -46.46 & & & -41.42 & -22.73 & -26.52 & -23.09 & -28.98 & & \\
\hline \multicolumn{6}{|c|}{ Industry-Level Effect (average of High-Low column; Alpha column) } & 29.63 & 30.29 & & & & & & 11.48 & 11.93 \\
\hline \multicolumn{6}{|c|}{ Stock-Level Effect (average of High-Low row; Alpha row) } & -45.28 & -44.70 & & & & & & -29.35 & -28.55 \\
\hline
\end{tabular}


Table 12. Cont

\begin{tabular}{|c|c|c|c|c|c|c|c|c|c|c|c|c|c|c|}
\hline \multirow[b]{3}{*}{ Stock-Level Quintile } & \multicolumn{6}{|c|}{ Equal-Weighted Returns and Alphas } & \multicolumn{8}{|c|}{ Value-Weighted Returns and Alphas } \\
\hline & \multicolumn{6}{|c|}{ Industry-Level Quintile } & \multicolumn{8}{|c|}{ Industry-Level Quintile } \\
\hline & 1 (Low) & 2 & 3 & 4 & 5 (High) & High-Low & Alpha & 1 (Low) & 2 & 3 & 4 & 5 (High) & High-Low & Alpha \\
\hline \multicolumn{15}{|l|}{ Part II: $t$-Statistics } \\
\hline 1 (Low) & 3.82 & 4.37 & 4.98 & 5.87 & 6.83 & 5.20 & 5.35 & 4.61 & 3.98 & 3.93 & 4.55 & 4.71 & 1.40 & 1.27 \\
\hline 2 & 2.90 & 3.13 & 3.49 & 4.48 & 5.22 & 3.50 & 3.35 & 3.82 & 3.56 & 2.35 & 4.58 & 3.16 & 1.14 & 1.00 \\
\hline 3 & 1.39 & 2.09 & 2.28 & 3.11 & 4.43 & 4.60 & 4.53 & 2.34 & 3.23 & 2.25 & 2.76 & 1.87 & 1.54 & 1.73 \\
\hline 4 & 0.70 & 1.28 & 1.60 & 2.12 & 3.93 & 4.40 & 4.47 & 1.69 & 1.62 & 1.36 & 1.87 & 0.68 & 1.50 & 1.54 \\
\hline 5 (High) & -0.79 & 0.22 & 1.07 & 1.88 & 3.16 & 5.88 & 5.93 & -0.51 & 1.27 & 0.62 & 1.93 & -1.00 & 2.82 & 3.06 \\
\hline Alpha, FFC4 & -9.00 & -8.16 & -7.97 & -7.93 & -8.34 & & & -7.37 & -3.73 & -5.06 & -4.31 & -5.47 & & \\
\hline \multirow{2}{*}{\multicolumn{6}{|c|}{ Industry-Level Effect (average of High-Low column; Alpha column) }} & 5.66 & 5.57 & & & & & & 2.23 & 2.20 \\
\hline & & & & & & -11.39 & -11.50 & & & & & & -8.88 & -8.91 \\
\hline
\end{tabular}

Notes: See notes to Table 8 . This table presents average returns and risk-adjusted alphas for portfolios sorted by stock-level and industry-level SRVJ. The sample includes all NYSE, NASDAQ and AMEX listed stocks for the period January 1993 to December 2016. A stock's industry signed jump variation (SRVJ) is the capitalization-weighted average of the SRV of all stocks within the industry. At the end of each Tuesday, all stocks in the sample are sorted into quintile portfolios based on stock-level and industry-level SRVJ, independently, resulting in 25 portfolios. Each portfolio is held for one week. The row labeled "Industry-Level Effect" reports average values of one-week ahead returns (and Fama-French-Carhart four-factor alphas in the High-Low (Alpha) column) in Part I (corresponding Newey-West $t$-statistics are given in Part II). The row labeled "Stock-Level Effect" reports the average values of one-week-ahead returns (and alphas) in Part I (corresponding Newey-West $t$-statistics are again given in Part II).

Table 13. Double-Sorted Portfolios: Portfolios Sorted by Stock- and Industry-Level SRVLJ/SRVSJ Independently.

\begin{tabular}{|c|c|c|c|c|c|c|c|c|c|c|c|c|c|c|}
\hline \multicolumn{15}{|c|}{ Panel A: Portfolios Sorted Based on SRVLJ } \\
\hline & \multicolumn{6}{|c|}{ Equal-Weighted Returns and Alphas } & \multicolumn{8}{|c|}{ Value-Weighted Returns and Alphas } \\
\hline & \multicolumn{6}{|c|}{ Industry-Level Quintile } & \multicolumn{8}{|c|}{ Industry-Level Quintile } \\
\hline Stock-Level Quintile & 1 (Low) & 2 & 3 & 4 & 5 (High) & High-Low & Alpha & 1 (Low) & 2 & 3 & 4 & 5 (High) & High-Low & Alpha \\
\hline \multicolumn{15}{|c|}{ Part I: Mean Return and Alpha } \\
\hline 1 (Low) & 34.64 & 35.34 & 35.04 & 51.12 & 51.00 & 16.36 & 17.03 & 22.26 & 24.61 & 20.46 & 26.39 & 25.19 & 2.92 & 0.73 \\
\hline 2 & 25.37 & 25.36 & 22.38 & 34.21 & 36.00 & 10.64 & 9.43 & 18.78 & 22.97 & 19.49 & 23.51 & 27.29 & 8.51 & 7.46 \\
\hline 3 & 17.55 & 0.89 & 26.31 & 34.26 & 31.56 & 14.40 & 11.15 & 18.90 & 15.95 & 11.59 & 4.81 & 17.06 & -6.76 & -10.28 \\
\hline 5 (High) & 12.22 & 11.90 & 14.43 & 25.57 & 25.57 & 13.36 & 12.93 & 14.16 & 21.60 & 16.13 & 20.63 & 22.85 & 8.69 & 11.30 \\
\hline High-Low & -22.42 & -23.44 & -20.61 & -25.55 & -25.42 & & & -8.10 & -3.00 & -4.33 & -5.75 & -2.33 & & \\
\hline Alpha & -22.02 & -23.37 & -20.45 & -25.74 & -26.12 & & & -9.58 & -3.52 & -4.34 & -5.40 & 1.00 & & \\
\hline \multicolumn{6}{|c|}{ Industry-Level Effect (average of High-Low column; Alpha column) } & 14.83 & 14.31 & & & & & & 7.24 & 6.90 \\
\hline \multicolumn{6}{|c|}{ Stock-Level Effect (average of High-Low row; Alpha row) } & -23.49 & -23.54 & & & & & & -4.70 & -4.37 \\
\hline
\end{tabular}


Table 13. Cont

\begin{tabular}{|c|c|c|c|c|c|c|c|c|c|c|c|c|c|c|}
\hline \multicolumn{15}{|c|}{ Panel A: Portfolios Sorted Based on SRVLJ } \\
\hline & \multicolumn{6}{|c|}{ Equal-Weighted Returns and Alphas } & \multicolumn{8}{|c|}{ Value-Weighted Returns and Alphas } \\
\hline & \multicolumn{6}{|c|}{ Industry-Level Quintile } & \multicolumn{8}{|c|}{ Industry-Level Quintile } \\
\hline Stock-Level Quintile & 1 (Low) & 2 & 3 & 4 & 5 (High) & High-Low & Alpha & 1 (Low) & 2 & 3 & 4 & 5 (High) & High-Low & Alpha \\
\hline \multicolumn{15}{|l|}{ Part II: $t$-Statistics } \\
\hline 1 (Low) & 3.63 & 3.39 & 3.33 & 4.94 & 5.23 & 3.40 & 3.41 & 3.25 & 3.06 & 2.48 & 3.25 & 3.16 & 0.49 & 0.12 \\
\hline 2 & 2.55 & 2.44 & 2.14 & 3.46 & 3.73 & 2.17 & 1.90 & 2.70 & 3.17 & 2.57 & 3.20 & 3.73 & 1.60 & 1.32 \\
\hline 3 & 0.78 & 0.04 & 0.93 & 1.41 & 1.46 & 0.85 & 0.65 & 1.08 & 0.73 & 0.51 & 0.25 & 1.05 & -0.37 & -0.54 \\
\hline 5 (High) & 1.31 & 1.24 & 1.50 & 2.84 & 2.99 & 2.97 & 2.91 & 1.83 & 2.83 & 2.12 & 2.83 & 3.56 & 1.50 & 2.09 \\
\hline High-Low & -6.14 & -5.13 & -4.66 & -5.85 & -6.19 & & & -1.57 & -0.59 & -0.90 & -1.19 & -0.45 & & \\
\hline Alpha, FFC4 & -6.09 & -5.03 & -4.58 & -5.91 & -6.38 & & & -1.93 & -0.68 & -0.87 & -1.09 & 0.21 & & \\
\hline \multicolumn{6}{|c|}{ Industry-Level Effect (average of High-Low column; Alpha column) } & 3.77 & 3.55 & & & & & & 1.77 & 1.57 \\
\hline Stock-Level Effect (aver & age of Hig & h-Low rov & ; Alpha 1 & w) & & -9.05 & -9.14 & & & & & & -2.01 & -1.91 \\
\hline \multicolumn{15}{|c|}{ Panel B: Portfolios Sorted Based on SRVSJ } \\
\hline & \multicolumn{6}{|c|}{ Equal-Weighted Returns and Alphas } & \multicolumn{8}{|c|}{ Value-Weighted Returns and Alphas } \\
\hline & \multicolumn{6}{|c|}{ Industry-Level Quintile } & \multicolumn{8}{|c|}{ Industry-Level Quintile } \\
\hline \multicolumn{15}{|c|}{ Part I: Mean Return and Alpha } \\
\hline 1 (Low) & 40.39 & 42.50 & 43.55 & 55.02 & 68.45 & 28.07 & 29.88 & 44.09 & 37.87 & 32.07 & 36.14 & 45.53 & 1.44 & 0.72 \\
\hline 2 & 32.52 & 40.60 & 41.29 & 45.73 & 59.67 & 27.15 & 28.20 & 24.11 & 24.09 & 23.82 & 27.45 & 42.09 & 17.99 & 18.36 \\
\hline 3 & 15.39 & 22.96 & 24.90 & 30.69 & 45.29 & 29.90 & 30.43 & 20.22 & 15.93 & 21.91 & 17.63 & 25.37 & 5.16 & 4.88 \\
\hline 4 & 9.07 & 9.08 & 17.26 & 17.20 & 32.70 & 23.63 & 23.94 & 11.38 & 12.88 & 22.53 & 11.01 & 25.43 & 14.04 & 15.23 \\
\hline 5 (High) & -2.44 & 3.16 & 7.13 & 12.21 & 22.30 & 24.73 & 26.19 & 8.91 & 7.91 & 11.60 & 9.76 & 11.23 & 2.32 & 5.33 \\
\hline High-Low & -42.83 & -39.34 & -36.43 & -42.82 & -46.16 & & & -35.18 & -29.95 & -20.47 & -26.38 & -34.30 & & \\
\hline Alpha & -42.27 & -39.03 & -34.75 & -42.27 & -45.96 & & & -36.51 & -30.26 & -18.68 & -25.89 & -31.90 & & \\
\hline \multirow{2}{*}{\multicolumn{6}{|c|}{$\begin{array}{l}\text { Industry-Level Effect (average of High-Low column; Alpha column) } \\
\text { Stock-Level Effect (average of High-Low row; Alpha row) }\end{array}$}} & 26.69 & 27.73 & & & & & & 8.19 & 8.90 \\
\hline & & & & & & -41.51 & -40.86 & & & & & & -29.26 & -28.65 \\
\hline
\end{tabular}


Table 13. Cont

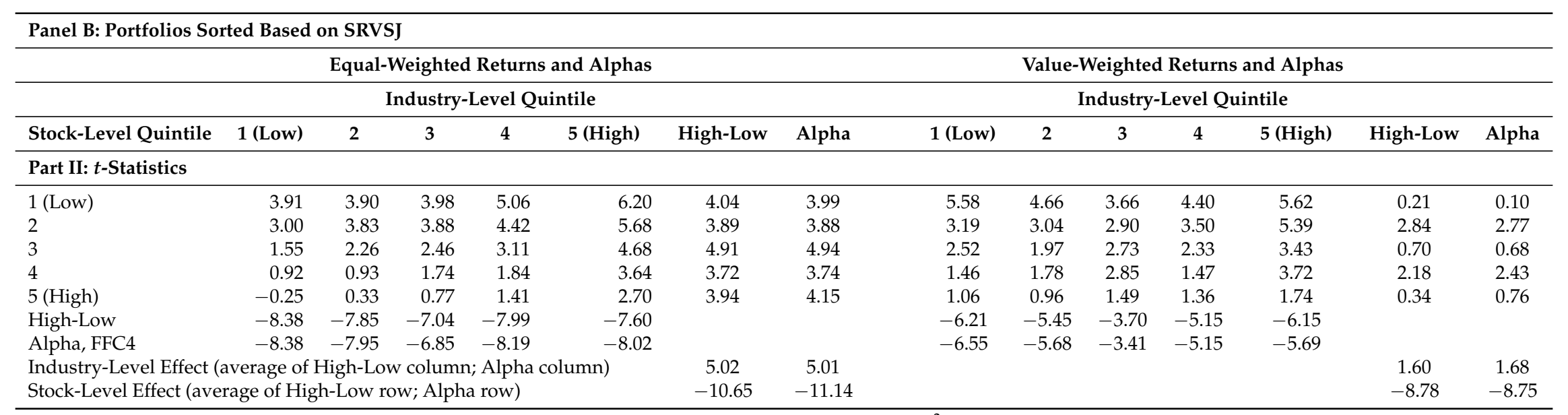

Notes: See notes to Table 12. Jumps are decomposed using truncation level $\gamma^{2}$, as discussed in the footnote to Table 2 . 
Table 14. Fama-MacBeth Cross-Sectional Regressions.

\begin{tabular}{|c|c|c|c|c|c|c|c|c|c|c|c|c|c|c|c|c|}
\hline \multicolumn{17}{|c|}{ Panel A: Regressions Without Control Variables } \\
\hline & I & II & III & IV & $\mathbf{V}$ & VI & VII & VIII & IX & $\mathrm{x}$ & XI & XII & XIII & XIV & $\mathbf{X V}$ & XVI \\
\hline Intercept & $\begin{array}{l}18.54 \\
(1.94)\end{array}$ & $\begin{array}{l}27.95 \\
(3.07)\end{array}$ & $\begin{array}{l}23.99 \\
(2.82)\end{array}$ & $\begin{array}{l}15.77 \\
(1.69)\end{array}$ & $\begin{array}{l}30.31 \\
(3.32)\end{array}$ & $\begin{array}{l}31.03 \\
(3.37)\end{array}$ & $\begin{array}{l}31.46 \\
(3.41)\end{array}$ & $\begin{array}{l}31.20 \\
(3.39)\end{array}$ & $\begin{array}{l}19.74 \\
(2.03)\end{array}$ & $\begin{array}{l}32.04 \\
(3.73)\end{array}$ & $\begin{array}{l}20.01 \\
(2.14)\end{array}$ & $\begin{array}{l}20.04 \\
(2.08)\end{array}$ & $\begin{array}{l}28.88 \\
(3.29)\end{array}$ & $\begin{array}{l}30.01 \\
(3.40)\end{array}$ & $\begin{array}{l}30.48 \\
(3.44)\end{array}$ & $\begin{array}{l}30.17 \\
(3.41)\end{array}$ \\
\hline RVJP & $\begin{array}{l}-63.86 \\
(-6.00)\end{array}$ & & & & & & & & $\begin{array}{l}-128.25 \\
(-6.24)\end{array}$ & & & & & & & \\
\hline RVJN & $\begin{array}{l}107.11 \\
(8.29)\end{array}$ & & & & & & & & $\begin{array}{c}196.57 \\
(8.98)\end{array}$ & & & & & & & \\
\hline RVLJP & & $\begin{array}{l}-53.42 \\
(-6.46)\end{array}$ & & $\begin{array}{l}-44.85 \\
(-4.46)\end{array}$ & & & & & & $\begin{array}{l}76.84 \\
(6.58)\end{array}$ & & $\begin{array}{l}-79.63 \\
(-3.94)\end{array}$ & & & & \\
\hline RVLJN & & $\begin{array}{l}71.27 \\
(8.12)\end{array}$ & & $\begin{array}{l}83.09 \\
(7.45)\end{array}$ & & & & & & $\begin{array}{l}-30.83 \\
(-2.40)\end{array}$ & & $\begin{array}{c}149.92 \\
(7.18)\end{array}$ & & & & \\
\hline RVSJP & & & $\begin{array}{l}-130.77 \\
(-8.97)\end{array}$ & $\begin{array}{l}-99.16 \\
(-6.24)\end{array}$ & & & & & & & $\begin{array}{l}-88.56 \\
(-6.64)\end{array}$ & $\begin{array}{l}-129.39 \\
(-6.33)\end{array}$ & & & & \\
\hline RVSJN & & & $\begin{array}{l}165.05 \\
(8.22)\end{array}$ & $\begin{array}{l}161.39 \\
(7.19)\end{array}$ & & & & & & & $\begin{array}{l}129.24 \\
(7.94)\end{array}$ & $\begin{array}{l}195.31 \\
(8.26)\end{array}$ & & & & \\
\hline SRVLJ & & & & & $\begin{array}{l}-50.07 \\
(-7.98)\end{array}$ & & $\begin{array}{l}-53.94 \\
(-8.37)\end{array}$ & & & & & & $\begin{array}{r}72.19 \\
(6.60)\end{array}$ & & $\begin{array}{l}-82.69 \\
(-4.48)\end{array}$ & \\
\hline SRVSJ & & & & & & $\begin{array}{l}-141.69 \\
(-9.25)\end{array}$ & $\begin{array}{l}-144.75 \\
(-9.32)\end{array}$ & & & & & & & $\begin{array}{l}-103.72 \\
(-8.25)\end{array}$ & $\begin{array}{l}-149.56 \\
(-7.66)\end{array}$ & \\
\hline SRVJ & & & & & & & & $\begin{array}{c}-81.15 \\
(-10.15)\end{array}$ & & & & & & & & $\begin{array}{l}-150.59 \\
(-7.80)\end{array}$ \\
\hline RVOL & & & & & & & & & $\begin{array}{c}-8.94 \\
(-1.60)\end{array}$ & $\begin{array}{c}-7.46 \\
(-1.32)\end{array}$ & $\begin{array}{c}-6.74 \\
(-1.21)\end{array}$ & $\begin{array}{c}-9.08 \\
(-1.62)\end{array}$ & $\begin{array}{c}-5.90 \\
(-1.05)\end{array}$ & $\begin{array}{l}-6.31 \\
(-1.12)\end{array}$ & $\begin{array}{c}-6.40 \\
(-1.14)\end{array}$ & $\begin{array}{l}-6.38 \\
(-1.13)\end{array}$ \\
\hline RSK & & & & & & & & & $\begin{array}{l}16.12 \\
(5.59)\end{array}$ & $\begin{array}{l}-22.16 \\
(-9.55)\end{array}$ & $\begin{array}{l}-9.87 \\
(-9.49)\end{array}$ & $\begin{array}{c}9.12 \\
(3.07)\end{array}$ & $\begin{array}{c}-24.75 \\
(-10.41)\end{array}$ & $\begin{array}{l}-10.15 \\
(-9.72)\end{array}$ & $\begin{array}{c}4.08 \\
(1.39)\end{array}$ & $\begin{array}{l}14.02 \\
(4.91)\end{array}$ \\
\hline RKT & & & & & & & & & $\begin{array}{c}-0.68 \\
(-2.25)\end{array}$ & $\begin{array}{l}-0.68 \\
(-2.27)\end{array}$ & $\begin{array}{c}0.46 \\
(1.45)\end{array}$ & $\begin{array}{l}-0.68 \\
(-2.24)\end{array}$ & $\begin{array}{c}0.12 \\
(0.42)\end{array}$ & $\begin{array}{c}0.09 \\
(0.30)\end{array}$ & $\begin{array}{c}0.08 \\
(0.28)\end{array}$ & $\begin{array}{c}0.09 \\
(0.32)\end{array}$ \\
\hline Adjusted $R^{2}$ & 0.0063 & 0.0033 & 0.0035 & 0.0082 & 0.0005 & 0.0019 & 0.0024 & 0.0016 & 0.0204 & 0.0175 & 0.0185 & 0.0214 & 0.0160 & 0.0168 & 0.0172 & 0.0168 \\
\hline
\end{tabular}


Table 14. Cont.

\begin{tabular}{|c|c|c|c|c|c|c|c|c|c|c|c|c|c|c|c|c|}
\hline \multicolumn{17}{|c|}{ Panel B: Regressions with Control Variables } \\
\hline & I & II & III & IV & $\mathrm{V}$ & VI & VII & VIII & IX & $x$ & XI & XII & XIII & XIV & $\mathbf{X V}$ & XVI \\
\hline Intercept & $\begin{array}{c}100.76 \\
(4.24)\end{array}$ & $\begin{array}{l}100.26 \\
(5.45)\end{array}$ & $\begin{array}{l}92.97 \\
(5.25)\end{array}$ & $\begin{array}{l}98.02 \\
(4.15)\end{array}$ & $\begin{array}{l}97.60 \\
(5.67)\end{array}$ & $\begin{array}{l}97.72 \\
(5.65)\end{array}$ & $\begin{array}{l}98.57 \\
(5.69)\end{array}$ & $\begin{array}{l}98.26 \\
(5.67)\end{array}$ & $\begin{array}{l}89.27 \\
(3.30)\end{array}$ & $\begin{array}{l}92.87 \\
(4.26)\end{array}$ & $\begin{array}{l}93.62 \\
(3.93)\end{array}$ & $\begin{array}{l}89.60 \\
(3.27)\end{array}$ & $\begin{array}{l}94.59 \\
(4.37)\end{array}$ & $\begin{array}{l}94.82 \\
(4.33)\end{array}$ & $\begin{array}{l}95.19 \\
(4.30)\end{array}$ & $\begin{array}{l}94.94 \\
(4.29)\end{array}$ \\
\hline RVJP & $\begin{array}{l}-30.35 \\
(-3.04)\end{array}$ & & & & & & & & $\begin{array}{l}-33.59 \\
(-1.87)\end{array}$ & & & & & & & \\
\hline RVJN & $\begin{array}{l}28.77 \\
(3.26)\end{array}$ & & & & & & & & $\begin{array}{l}50.58 \\
(3.48)\end{array}$ & & & & & & & \\
\hline RVLJP & & $\begin{array}{l}-27.56 \\
(-4.46)\end{array}$ & & $\begin{array}{l}-27.07 \\
(-2.81)\end{array}$ & & & & & & $\begin{array}{l}11.55 \\
(1.20)\end{array}$ & & $\begin{array}{l}-28.36 \\
(-1.53)\end{array}$ & & & & \\
\hline RVLJN & & $\begin{array}{l}16.42 \\
(2.67)\end{array}$ & & $\begin{array}{l}23.05 \\
(2.61)\end{array}$ & & & & & & $\begin{array}{c}-0.32 \\
(-0.03)\end{array}$ & & $\begin{array}{l}48.59 \\
(2.89)\end{array}$ & & & & \\
\hline RVSJP & & & $\begin{array}{l}-26.94 \\
(-2.78)\end{array}$ & $\begin{array}{l}-34.15 \\
(-2.51)\end{array}$ & & & & & & & $\begin{array}{l}-24.07 \\
(-2.20)\end{array}$ & $\begin{array}{l}-38.20 \\
(-2.04)\end{array}$ & & & & \\
\hline RVSJN & & & $\begin{array}{l}44.62 \\
(4.25)\end{array}$ & $\begin{array}{l}45.83 \\
(3.81)\end{array}$ & & & & & & & $\begin{array}{l}26.48 \\
(2.67)\end{array}$ & $\begin{array}{l}52.34 \\
(3.42)\end{array}$ & & & & \\
\hline SRVLJ & & & & & $\begin{array}{l}-22.63 \\
(-5.18)\end{array}$ & & $\begin{array}{l}-25.76 \\
(-5.67)\end{array}$ & & & & & & $\begin{array}{c}9.90 \\
(1.10)\end{array}$ & & $\begin{array}{l}-31.02 \\
(-1.93)\end{array}$ & \\
\hline SRVSJ & & & & & & $\begin{array}{l}-33.16 \\
(-3.92)\end{array}$ & $\begin{array}{l}-38.71 \\
(-4.45)\end{array}$ & & & & & & & $\begin{array}{l}-23.75 \\
(-2.74)\end{array}$ & $\begin{array}{l}-41.74 \\
(-2.83)\end{array}$ & \\
\hline SRVJ & & & & & & & & $\begin{array}{l}-28.64 \\
(-6.26)\end{array}$ & & & & & & & & $\begin{array}{l}-39.38 \\
(-2.69)\end{array}$ \\
\hline RVOL & & & & & & & & & $\begin{array}{c}4.79 \\
(0.79)\end{array}$ & $\begin{array}{c}5.07 \\
(0.84)\end{array}$ & $\begin{array}{c}4.59 \\
(0.76)\end{array}$ & $\begin{array}{c}4.68 \\
(0.77)\end{array}$ & $\begin{array}{c}4.94 \\
(0.82)\end{array}$ & $\begin{array}{c}4.87 \\
(0.80)\end{array}$ & $\begin{array}{c}4.86 \\
(0.80)\end{array}$ & $\begin{array}{c}4.84 \\
(0.79)\end{array}$ \\
\hline RSK & & & & & & & & & $\begin{array}{c}3.02 \\
(1.23)\end{array}$ & $\begin{array}{c}-4.90 \\
(-3.01)\end{array}$ & $\begin{array}{c}-3.58 \\
(-4.69)\end{array}$ & $\begin{array}{c}2.67 \\
(0.98)\end{array}$ & $\begin{array}{c}-5.53 \\
(-3.45)\end{array}$ & $\begin{array}{c}-3.67 \\
(-4.79)\end{array}$ & $\begin{array}{c}1.42 \\
(0.50)\end{array}$ & $\begin{array}{c}2.46 \\
(0.95)\end{array}$ \\
\hline RKT & & & & & & & & & $\begin{array}{c}-0.53 \\
(-2.00)\end{array}$ & $\begin{array}{c}-0.61 \\
(-2.27)\end{array}$ & $\begin{array}{c}-0.38 \\
(-1.08)\end{array}$ & $\begin{array}{c}-0.56 \\
(-1.87)\end{array}$ & $\begin{array}{c}-0.49 \\
(-1.78)\end{array}$ & $\begin{array}{c}-0.46 \\
(-1.63)\end{array}$ & $\begin{array}{c}-0.44 \\
(-1.49)\end{array}$ & $\begin{array}{r}-0.44 \\
(-1.46)\end{array}$ \\
\hline Beta & $\begin{array}{c}-8.28 \\
(-1.46)\end{array}$ & $\begin{array}{c}-8.11 \\
(-1.40)\end{array}$ & $\begin{array}{c}-8.09 \\
(-1.38)\end{array}$ & $\begin{array}{c}-8.27 \\
(-1.46)\end{array}$ & $\begin{array}{c}-8.07 \\
(-1.37)\end{array}$ & $\begin{array}{c}-8.26 \\
(-1.41)\end{array}$ & $\begin{array}{c}-8.29 \\
(-1.41)\end{array}$ & $\begin{array}{c}-8.16 \\
(-1.39)\end{array}$ & $\begin{array}{l}-7.71 \\
(-1.36)\end{array}$ & $\begin{array}{l}-7.75 \\
(-1.35)\end{array}$ & $\begin{array}{l}-8.18 \\
(-1.42)\end{array}$ & $\begin{array}{l}-7.72 \\
(-1.37)\end{array}$ & $\begin{array}{l}-8.10 \\
(-1.40)\end{array}$ & $\begin{array}{l}-8.18 \\
(-1.42)\end{array}$ & $\begin{array}{l}-8.22 \\
(-1.43)\end{array}$ & $\begin{array}{c}-8.14 \\
(-1.41)\end{array}$ \\
\hline $\log ($ Size $)$ & $\begin{array}{r}-14.93 \\
(-5.24)\end{array}$ & $\begin{array}{l}-14.77 \\
(-5.25)\end{array}$ & $\begin{array}{r}-14.73 \\
(-5.21)\end{array}$ & $\begin{array}{l}-14.94 \\
(-5.32)\end{array}$ & $\begin{array}{l}-14.76 \\
(-5.15)\end{array}$ & $\begin{array}{l}-14.76 \\
(-5.14)\end{array}$ & $\begin{array}{l}-14.67 \\
(-5.13)\end{array}$ & $\begin{array}{l}-14.67 \\
(-5.13)\end{array}$ & $\begin{array}{r}-14.36 \\
(-5.19)\end{array}$ & $\begin{array}{l}1.25 \\
-14.25 \\
(-5.15)\end{array}$ & $\begin{array}{r}-14.29 \\
(-5.16)\end{array}$ & $\begin{array}{l}-14.48 \\
(-5.26)\end{array}$ & $\begin{array}{r}-14.13 \\
(-5.08)\end{array}$ & $\begin{array}{l}-14.11 \\
(-5.07)\end{array}$ & $\begin{array}{l}-14.04 \\
(-5.06)\end{array}$ & $\begin{array}{l}-14.05 \\
(-5.07)\end{array}$ \\
\hline $\mathrm{BE} / \mathrm{ME}$ & $\begin{array}{l}-0.76 \\
(-0.37)\end{array}$ & $\begin{array}{c}-0.75 \\
(-0.36)\end{array}$ & $\begin{array}{c}-0.65 \\
(-0.32)\end{array}$ & $\begin{array}{l}-0.76 \\
(-0.37)\end{array}$ & $\begin{array}{c}-0.67 \\
(-0.33)\end{array}$ & $\begin{array}{l}-0.61 \\
(-0.30)\end{array}$ & $\begin{array}{c}-0.59 \\
(-0.29)\end{array}$ & $\begin{array}{c}-0.62 \\
(-0.30)\end{array}$ & $\begin{array}{c}-0.72 \\
(-0.34)\end{array}$ & $\begin{array}{l}-0.57 \\
(-0.27)\end{array}$ & $\begin{array}{c}-0.57 \\
(-0.27)\end{array}$ & $\begin{array}{c}-0.69 \\
(-0.33)\end{array}$ & $\begin{array}{l}-0.56 \\
(-0.27)\end{array}$ & $\begin{array}{c}-0.54 \\
(-0.26)\end{array}$ & $\begin{array}{l}-0.56 \\
(-0.27)\end{array}$ & $\begin{array}{c}-0.58 \\
(-0.28)\end{array}$ \\
\hline MOM & $\begin{array}{c}0.00 \\
(0.96)\end{array}$ & $\begin{array}{c}0.00 \\
(0.97)\end{array}$ & $\begin{array}{c}0.00 \\
(0.94)\end{array}$ & $\begin{array}{c}0.00 \\
(0.98)\end{array}$ & $\begin{array}{c}0.00 \\
(0.93)\end{array}$ & $\begin{array}{c}0.00 \\
(0.93)\end{array}$ & $\begin{array}{c}0.00 \\
(0.93)\end{array}$ & $\begin{array}{c}0.00 \\
(0.93)\end{array}$ & $\begin{array}{c}0.00 \\
(1.26)\end{array}$ & $\begin{array}{c}0.00 \\
(1.19)\end{array}$ & $\begin{array}{c}0.00 \\
(1.18)\end{array}$ & $\begin{array}{c}0.00 \\
(1.22)\end{array}$ & $\begin{array}{c}0.00 \\
(1.19)\end{array}$ & $\begin{array}{c}0.00 \\
(1.21)\end{array}$ & $\begin{array}{c}0.00 \\
(1.20)\end{array}$ & $\begin{array}{c}0.00 \\
(1.21)\end{array}$ \\
\hline REV & $\begin{array}{c}-0.01 \\
(-5.63)\end{array}$ & $\begin{array}{c}-0.01 \\
(-5.83)\end{array}$ & $\begin{array}{c}-0.01 \\
(-5.63)\end{array}$ & $\begin{array}{c}-0.01 \\
(-5.53)\end{array}$ & $\begin{array}{c}-0.01 \\
(-5.84)\end{array}$ & $\begin{array}{c}-0.01 \\
(-5.66)\end{array}$ & $\begin{array}{c}-0.01 \\
(-5.55)\end{array}$ & $\begin{array}{c}-0.01 \\
(-5.64)\end{array}$ & $\begin{array}{c}-0.01 \\
(-5.74)\end{array}$ & $\begin{array}{l}-0.01 \\
(-5.97)\end{array}$ & $\begin{array}{c}-0.01 \\
(-5.82)\end{array}$ & $\begin{array}{c}-0.01 \\
(-5.70)\end{array}$ & $\begin{array}{c}-0.01 \\
(-5.99)\end{array}$ & $\begin{array}{c}-0.01 \\
(-5.83)\end{array}$ & $\begin{array}{c}-0.01 \\
(-5.77)\end{array}$ & $\begin{array}{l}-0.01 \\
(-5.80)\end{array}$ \\
\hline IVOL & $\begin{array}{l}-301.46 \\
(-2.18)\end{array}$ & $\begin{array}{l}-293.89 \\
(-2.15)\end{array}$ & $\begin{array}{l}-297.02 \\
(-2.17)\end{array}$ & $\begin{array}{l}-304.39 \\
(-2.21)\end{array}$ & $\begin{array}{l}-291.92 \\
(-2.13)\end{array}$ & $\begin{array}{l}-292.47 \\
(-2.12)\end{array}$ & $\begin{array}{l}-298.32 \\
(-2.16)\end{array}$ & $\begin{array}{l}-298.14 \\
(-2.17)\end{array}$ & $\begin{array}{l}-425.97 \\
(-4.69)\end{array}$ & $\begin{array}{l}-416.77 \\
(-4.57)\end{array}$ & $\begin{array}{l}-422.83 \\
(-4.63)\end{array}$ & $\begin{array}{l}-424.54 \\
(-4.67)\end{array}$ & $\begin{array}{l}-419.05 \\
(-4.59)\end{array}$ & $\begin{array}{l}-421.52 \\
(-4.62)\end{array}$ & $\begin{array}{l}-424.24 \\
(-4.65)\end{array}$ & $\begin{array}{l}-425.20 \\
(-4.67)\end{array}$ \\
\hline CSK & $\begin{array}{c}-7.52 \\
(-1.75)\end{array}$ & $\begin{array}{c}-8.67 \\
(-2.01)\end{array}$ & $\begin{array}{c}-8.13 \\
(-1.89)\end{array}$ & $\begin{array}{c}-7.29 \\
(-1.70)\end{array}$ & $\begin{array}{c}-8.69 \\
(-2.01)\end{array}$ & $\begin{array}{c}-8.23 \\
(-1.91)\end{array}$ & $\begin{array}{c}-7.62 \\
(-1.77)\end{array}$ & $\begin{array}{c}-7.87 \\
(-1.82)\end{array}$ & $\begin{array}{c}-7.00 \\
(-1.66)\end{array}$ & $\begin{array}{c}-7.84 \\
(-1.86)\end{array}$ & $\begin{array}{c}-7.44 \\
(-1.77)\end{array}$ & $\begin{array}{c}-6.89 \\
(-1.64)\end{array}$ & $\begin{array}{c}-7.91 \\
(-1.88)\end{array}$ & $\begin{array}{c}-7.45 \\
(-1.77)\end{array}$ & $\begin{array}{c}-7.35 \\
(-1.74)\end{array}$ & $\begin{array}{c}-7.52 \\
(-1.78)\end{array}$ \\
\hline CKT & 2.34 & 2.29 & 2.24 & 2.36 & 2.32 & 2.32 & 2.43 & 2.38 & 1.74 & 1.72 & 1.69 & 1.74 & 1.71 & 1.77 & 1.83 & 1.81 \\
\hline
\end{tabular}


Table 14. Cont.

\begin{tabular}{|c|c|c|c|c|c|c|c|c|c|c|c|c|c|c|c|c|}
\hline \multicolumn{17}{|c|}{ Panel B: Regressions with Control Variables } \\
\hline & I & II & III & IV & $\mathbf{v}$ & VI & VII & VIII & IX & $x$ & XI & XII & XIII & XIV & $\mathbf{X V}$ & XVI \\
\hline & (1.19) & $(1.15)$ & (1.13) & $(1.20)$ & (1.15) & (1.16) & $(1.21)$ & (1.18) & $(0.91)$ & $(0.89)$ & $(0.87)$ & $(0.91)$ & $(0.88)$ & $(0.91)$ & $(0.94)$ & $(0.93)$ \\
\hline MAX & $\begin{array}{c}-0.03 \\
(-5.33)\end{array}$ & $\begin{array}{c}-0.03 \\
(-5.66)\end{array}$ & $\begin{array}{c}-0.03 \\
(-5.57)\end{array}$ & $\begin{array}{c}-0.03 \\
(-5.31)\end{array}$ & $\begin{array}{c}-0.03 \\
(-5.64)\end{array}$ & $\begin{array}{c}-0.03 \\
(-5.71)\end{array}$ & $\begin{array}{c}-0.03 \\
(-5.55)\end{array}$ & $\begin{array}{c}-0.03 \\
(-5.54)\end{array}$ & $\begin{array}{c}-0.03 \\
(-7.37)\end{array}$ & $\begin{array}{c}-0.03 \\
(-7.55)\end{array}$ & $\begin{array}{c}-0.03 \\
(-7.45)\end{array}$ & $\begin{array}{c}-0.03 \\
(-7.35)\end{array}$ & $\begin{array}{c}-0.03 \\
(-7.59)\end{array}$ & $\begin{array}{c}-0.03 \\
(-7.55)\end{array}$ & $\begin{array}{c}-0.03 \\
(-7.57)\end{array}$ & $\begin{array}{c}-0.03 \\
(-7.55)\end{array}$ \\
\hline MIN & $\begin{array}{l}-0.02 \\
(-2.79)\end{array}$ & $\begin{array}{l}-0.02 \\
(-3.11)\end{array}$ & $\begin{array}{l}-0.02 \\
(-3.14)\end{array}$ & $\begin{array}{l}-0.02 \\
(-2.79)\end{array}$ & $\begin{array}{l}-0.02 \\
(-3.11)\end{array}$ & $\begin{array}{l}-0.02 \\
(-3.12)\end{array}$ & $\begin{array}{l}-0.02 \\
(-2.83)\end{array}$ & $\begin{array}{l}-0.02 \\
(-2.85)\end{array}$ & $\begin{array}{l}-0.01 \\
(-2.81)\end{array}$ & $\begin{array}{c}-0.02 \\
(-2.94)\end{array}$ & $\begin{array}{l}-0.01 \\
(-2.80)\end{array}$ & $\begin{array}{c}-0.01 \\
(-2.81)\end{array}$ & $\begin{array}{l}-0.01 \\
(-2.88)\end{array}$ & $\begin{array}{l}-0.01 \\
(-2.80)\end{array}$ & $\begin{array}{l}-0.01 \\
(-2.73)\end{array}$ & $\begin{array}{l}-0.01 \\
(-2.75)\end{array}$ \\
\hline ILLIQ & $\begin{array}{c}-7.84 \\
(-5.24)\end{array}$ & $\begin{array}{c}-7.68 \\
(-5.12)\end{array}$ & $\begin{array}{c}-8.08 \\
(-5.22)\end{array}$ & $\begin{array}{l}-7.86 \\
(-5.26)\end{array}$ & $\begin{array}{c}-7.99 \\
(-5.15)\end{array}$ & $\begin{array}{c}-8.03 \\
(-5.16)\end{array}$ & $\begin{array}{c}-7.87 \\
(-5.08)\end{array}$ & $\begin{array}{c}-7.88 \\
(-5.10)\end{array}$ & $\begin{array}{l}-8.94 \\
(-4.79)\end{array}$ & $\begin{array}{l}-8.87 \\
(-5.12)\end{array}$ & $\begin{array}{l}-8.68 \\
(-4.96)\end{array}$ & $\begin{array}{c}-9.10 \\
(-4.87)\end{array}$ & $\begin{array}{l}-8.54 \\
(-4.97)\end{array}$ & $\begin{array}{l}-8.51 \\
(-4.95)\end{array}$ & $\begin{array}{l}-8.41 \\
(-4.86)\end{array}$ & $\begin{array}{c}-8.43 \\
(-4.88)\end{array}$ \\
\hline Adjusted $R^{2}$ & 0.0602 & 0.0597 & 0.0597 & 0.0609 & 0.0590 & 0.0592 & 0.0594 & 0.0592 & 0.0647 & 0.0641 & 0.0642 & 0.0652 & 0.0636 & 0.0637 & 0.0639 & 0.0638 \\
\hline
\end{tabular}

Notes: See notes to Tables 1 and 5. This table reports results for cross-sectional Fama-MacBeth regressions, based on the regression model depicted as Equation (15) in Section 4.5. In these regression models, future weekly returns are regressed on various realized measures and control variates. The two panels use jump truncation level $\gamma^{2}$, as discussed in the footnote to Table 2. The regressions that are reported on are of the form: $r_{i, t+1}=\gamma_{0, t}+\sum_{j=1}^{K_{1}} \gamma_{j, t} X_{i, j, t}+\sum_{s=1}^{K_{2}} \phi_{s, t} Z_{i, s, t}+\epsilon_{i, t+1}, t=1, \ldots, T$, where $r_{i, t+1}$ denotes the stock return for firm $i$ in week $t+1, K_{1}$ is the number of potential variation measures, and $X_{i, j, t}$ denotes a relevant realized measure at the end of week $t$. In addition, there are $K_{2}$ variables measuring firm characteristics, which are denoted by $Z_{i, j, t}$ (see Section 3 for details). In the table, time series averages of the coefficient estimates $\left(\frac{1}{T} \sum_{t=1}^{T} \widehat{\gamma}_{j, t}\right.$ and $\left.\frac{1}{T} \sum_{t=1}^{T} \widehat{\phi}_{j, t}\right)$ are reported, along with Newey-West $t$-statistics (in parentheses). For complete details, see Section 4. 


\subsection{Single (Univariate) Portfolio Sorts Based on Realized Measures}

In this section, we first discuss the results contained in Table 4. Recall that the Mean Return in this table is an average taken over our entire time series of equal-weighted and value-weighted portfolio returns, for single-sorted portfolios based on positive jump variation (RVJP), negative jump variation (RVJN) and signed jump variation (SRVJ). Values in parentheses are Newey-West $t$-statistics (see Bollerslev et al. (2015) and Petersen (2009) for further discussion). Panel A provides results for portfolios sorted by RVJP. Inspection of the entries in this panel indicates that mean returns and alphas of high-low portfolios (i.e., the difference in returns (alphas) between the fifth and first quintiles) are all negative, indicating a negative association between RVJP and subsequent stock returns. Interestingly, the alpha of -7.71 basis points (bps) is insignificant for the high-low spread for the equal-weighted portfolio, while the alpha of -5.63 bps is only significant at a $10 \%$ level for the high-low spread for the value weighted portfolio.

The lack of statistical significance for some of the mean return values reported in Panel A does not characterize our findings when negative and signed jump variation measures are used for sorting. Moreover, the magnitudes of the mean returns and alphas are usually three or more times larger when sorting on negative and signed jump variation (to see this, turn to Panels B and C of Table 4). In Panel B, the high-low spread of mean returns equals 36.06 bps, with a $t$-statistic of 6.47 for the equal-weighted portfolio, and $15.13 \mathrm{bps}$ with a $t$-statistic of 3.75 for the value-weighted portfolio. Moreover, both equal-weighted and value-weighted portfolios generate significant positive abnormal future returns measured by the alphas. These results clearly point to a statistically significant positive association between negative jump variation and the following week's returns.

Panel $C$ in Table 4 contains results for portfolios sorted by signed jump variation. The negative high-low spreads indicate a statistically significant negative association between signed jump variation and future returns. In particular, a strategy buying stocks in the lowest signed jump variation quintile and selling stocks in the highest signed jump variation quintile earns a mean return of 40.82 bps with a $t$-statistic of 9.85 each week for the equal-weighted portfolio and $25.02 \mathrm{bps}$ with a $t$-statistic of 5.78 for the value-weighted portfolio. These results are consistent with the results reported in Bollerslev et al. (2020). Interestingly, almost all of the mean returns listed in Table 4 are "alpha" (see tabulated average alphas in the table), and cannot be explained by standard portfolio risk factors using regressions of the type given above as Equation (14).

A key question that we provide evidence on in this paper is whether the results summarized in Table 4 carry over to the case where small and large jump variation is separately sorted on. First, consider large jumps. Table 5 reports the results for portfolios sorted by positive, negative and signed large jump variation, respectively. Similar to positive jump variation, positive large jumps negatively predict subsequent returns, but the predictability is not significant, regardless of the truncation level $(\gamma)$ used to separate small and large jumps, and regardless of portfolio weighting used. This is demonstrated by the fact that the $t$-statistics for mean returns and alphas of high-low portfolios all indicate insignificance, at a 5\% testing level, regardless of truncation level. Thus, there is no ambiguity, as in Panel A of Table 4. Positive jump variation is not a significant predictor, under our large jump scenario. On the other hand, we shall see that sorting on small and large negative variation measures yields significant excess returns, as does sorting on small positive jump variation, under both equal and value weighting schemes.

As just noted, equal-weighted high-low portfolios sorted on large negative jump variation generate significant positive returns and alphas (see Panel B of Table 5). However, analogous returns and alphas under value weighting are not significant. Signed large jump variation is sorted on in Panel C of Table 5. Signed large jump variation is useful for undertaking a long-short trading strategy based on the difference between large upside and downside jump variation measures. Inspection of the results in this panel of the table reveals that the high-low spread for the equal-weighted portfolio generates an average risk-adjusted weekly return of -28.36 bps (with a $t$-statistics of -9.39 ) and -9.25 bps (with a $t$-statistics of -2.87 ) for the value-weighted portfolio, for truncation level equal to $\gamma^{1}$. 
Results based on $\gamma^{2}$ (i.e., our larger truncation level) are also significant, although magnitudes are lesser and only for our equal-weighted portfolio. ${ }^{16}$ In particular, observe that when large jump variation is constructed using $\gamma^{2}$, the high-low spreads for value-weighted portfolios sorted by downside or signed large jump variation measures are insignificant, suggesting that small firms have stronger relationships (than larger firms) between signed (or negative) large jump variation and subsequent returns. This may be because smaller firms are in some ways more susceptible to changing market conditions than larger firms.

Table 6 summarizes results analogous to those reported in Table 5, but for positive, negative and signed small jump variation measures. Similar to large jump measures, positive and signed small jump variation measures negatively predict future returns, and negative small jump variation measures positively predict returns in the following week. By contrast, the differences in average (risk-adjusted) returns between equal-weighted and value-weighted long-short portfolios based on RVSJP and RVSJN are smaller than those for portfolios based on large jumps (compare the entries for the high-low quintiles under the two weighting schemes in Panels A and B of Table 6 with like entries in Panels A and B of Table 5). These results indicate that big firms have a stronger relationship between small jump variation and future returns than that between large jumps and subsequent weekly returns. Since stocks for big firms are more liquid and price discovery is more rapid, predictability associated with large jumps is much weaker or insignificant for big firms. This finding is in line with Bollerslev et al. (2020), who document that the predictability of signed jump variation is stronger for small and illiquid firms and is driven by investor overreaction. In addition, when using our larger truncation level, $\gamma^{2}$, value-weighted high-low spreads based on signed small jump variation are larger than those based on signed total jump variation and signed large jump variation. This result implies that a long-short strategy associated with signed small jump variation generates the highest value-weighted risk-adjusted returns, given the use of an appropriate truncation level to separate small and large jumps.

As discussed above, in order to understand why small jumps matter in our analysis, note that Scaillet et al. (2018) document that jumps are frequent events and that jump dynamics are not consistent with the compound Poisson processes with constant intensity. In addition, many recent studies find that infinite-activity Levy jump specifications (e.g., Variance Gamma and Normal Inverse Gaussian return jumps) can improve the goodness of fit and option pricing performance of models (for example, see Yang and Kanniainen (2016)). These results point to the possible importance of small jumps, which may have infinite numbers in a finite time interval. Though more frequent than large jumps, note that we estimate daily jump variation measures and aggregate them into weekly measures for forecasting future weekly returns. Thus, it is not individual small jumps that necessarily matter, but the overall contribution of small jumps to total variation, in the cross-section. Additionally, we show (see below discussion) that small jump variation is more closely associated with idiosyncratic risk than are large jump variation. If idiosyncratic volatility matters (see Ang et al. $(2006,2009)$ ), so do small jumps, at least to some extent. Indeed, our results partially help explain the idiosyncratic volatility puzzle (investors prefer positive skewness/asymmetry, so they will accept lower returns for stocks with a higher probability of having positive jumps).

Table 7 reports results for portfolios sorted by realized volatility, realized skewness, realized kurtosis, and continuous variance. Consistent with the results in Amaya et al. (2015) and Bollerslev et al. (2020), there is a significant negative relationship between realized skewness and future returns, while the association is not significant between either realized volatility or realized kurtosis and returns in the following week, regardless of portfolio weighting scheme. In addition,

16 Empirical findings based on $\gamma^{3}$ are similar to those discussed above, and hence are not reported. This robustness of our findings to the choice of $\gamma$ also characterizes the other empirical findings discussed in the sequel. 
continuous variance significantly and negatively predicts one-week-ahead returns for equal-weighted portfolios, but this negative association is not significant for value-weighted portfolios.

\subsection{Cumulative Returns and Sharpe Ratios}

Not surprisingly, our findings based on univariate portfolio sorts suggest that strategies that use different realized measures deliver different risk-adjusted average returns. In order to investigate this result further, we calculate cumulative returns and Sharpe ratios for short-long portfolios, sorted on various risk measures that are described in Table 1, including SRVJ, RSJ, SRVLJ, SRVSJ, and RSK. In addition, for comparison purposes, we also carry out our analysis using the relative signed jump variation measure (called RSJ) that is examined by Bollerslev et al. (2020). Our experiments are carried out as follows. Beginning in January 1993, various short-long portfolios are constructed, with an initial investment of $\$ 1$. These portfolios are re-balanced and accumulated at a weekly frequency, until the end of 2016. ${ }^{17}$ Figure 3 plots portfolio values over time. Consistent with our results based on single portfolio sorts, inspection of the plots in this figure indicates that for equal-weighted portfolios sorting on signed jump variation (SRVJ) yields the largest portfolio accumulations; and for value-weighted portfolios, sorting on signed small jump variation (SRVSJ) yields the largest portfolio accumulations. ${ }^{18}$
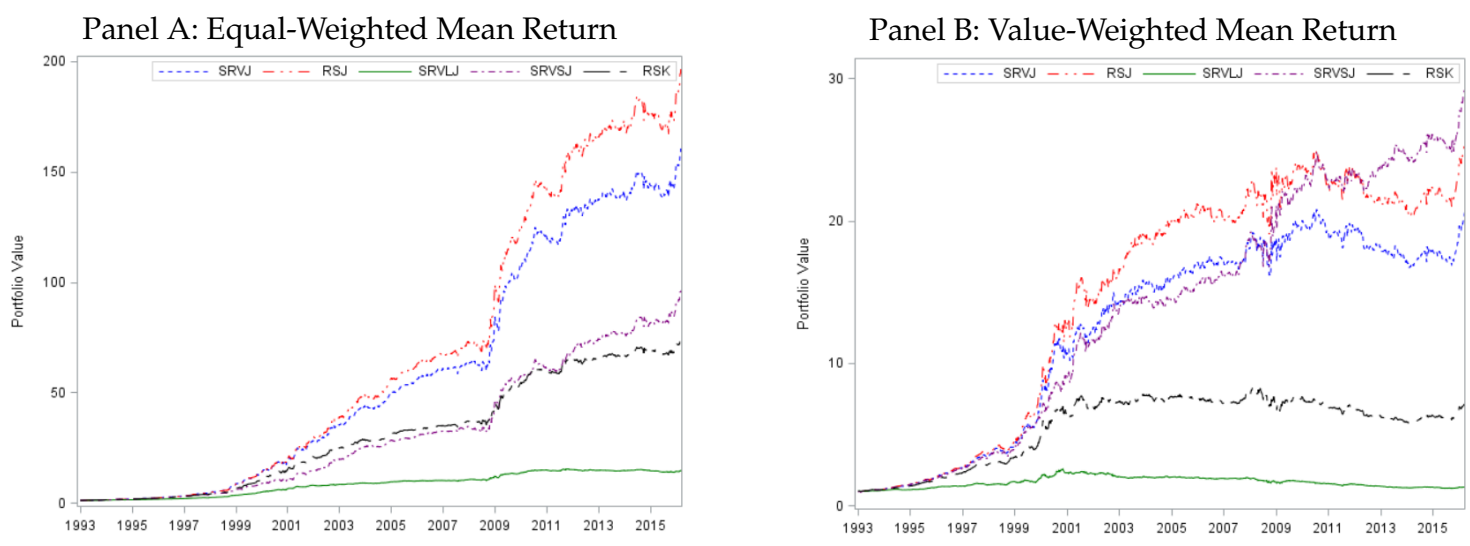

Figure 3. Cumulative Gains of Short-Long Portfolios. Panels A-B display cumulative gains of equal-weighted and value-weighted short-long portfolios constructed using SRVJ, SRVLJ, SRVSJ, and RSK (see Table 1 and Section 2 for a discussion of these measures). RSJ is the relative signed jump variation measure defined and analyzed in Bollerslev et al. (2020), who include the risk-free rate in all of their calculations, while we do not (refer to Bollerslev et al. (2020) for complete details). In all experiments, the initial investment, made on January 1993, is $\$ 1$. Each portfolio is re-balanced and accumulated on a weekly basis, through 2016. Signed small and large jump variation measures used in the experiment reported on in this figure are constructed based on truncation level $\gamma^{2}=5 \sqrt{\frac{1}{t} \widehat{I V}_{t}^{(i)}} \Delta_{n}^{0.49}$. See Section 4.2 for further discussion.

Now, consider the Sharpe ratios reported below, which are reported for various jump measures, and are constructed based on truncation level $\gamma^{2}=5 \sqrt{\frac{1}{t} \widehat{I V}_{t}^{(i)}} \Delta_{n}^{0.49}$.

17 Cumulative returns calculations do not include the risk-free rate. For a definition of cumulative returns both with and without the weekly risk-free rate, see Bollerslev et al. (2020).

18 Please note that RSJ, which measures the same signed jump variation as SRVI, although using different estimation methodology, generates the highest cumulative return for equal-weighted portfolios, but is dominated by SRVSJ for value-weighted portfolios. 
Table 15. Sharpe Ratios.

\begin{tabular}{lccccc}
\hline & SRVJ & RSJ & SRVLJ & SRVSJ & RSK \\
\hline Equal-Weighted & 2.1342 & 2.1363 & 1.8556 & 1.8161 & 2.2234 \\
Value-Weighted & 1.1322 & 1.1310 & 0.1611 & 1.2755 & 0.8665 \\
\hline
\end{tabular}

The entries in Table 15 are Sharpe ratios for equal- and value-weighted short-long portfolios constructed using SRVJ, RSJ, SRVLJ, SRVSJ, and RSK. Recall that RSK is realized skewness (see Table 1 for definitions of these measures). The sample of stocks used for Sharpe ratio calculations includes all NYSE, NASDAQ and AMEX listed stocks for the period January 1993 to December 2016. At the end of each Tuesday, all the stocks in the sample are sorted into quintile portfolios based on ascending values of various realized risk measures. A high-low spread portfolio is then formed as the difference between portfolio 1 and portfolio 5, and held for one week, where 1 and 5 refer to quintiles, as in Tables 3-7. The Sharpe ratio is calculated with the one-week-ahead returns.

Interestingly, for equal-weighted portfolios, the RSK-based short-long strategy yields the highest Sharpe ratio (i.e., 2.2234), although the ratio of 2.1342 for SRVJ is approximately the same. Still, the success of the RSK measure is likely due to its relatively stable performance, compared with other jump-based strategies. This finding is similar to the findings discussed in Xiong et al. (2016), who show that tail risks can be substantially reduced by forecasting skewness. Note also that the signed small jump variation (SRVSJ)-based portfolio has the highest Sharpe ratio, among all value-weighted portfolios. However, it is clear that all equal-weighted portfolios outperform their corresponding value-weighted counterparts. This result is consistent with the finding discussed above that small and illiquid firms tend to react more strongly to realized risk measures.

\subsection{Double Portfolio Sorts Based on Realized Measures}

To further investigate whether small and large jumps are priced differently, we use double portfolio sorts. In particular, we carry out double-sorts in order to examine the robustness of our findings based on single sorts, after controlling for other realized measures. Table 8 reports returns and alphas from various of these sorts in which we alternate the sorting order among SRVJ, SRVLJ and SRVSJ. When we first sort by total jump variation, and then sort stocks based on SRVLJ or SRVSJ, a negative relation only exists between SRVSJ and subsequent weekly returns (see Panels A and B of the table). This result indicates that there is no marginal predictive content associated with large jumps, when conditioning on the predictive content associated with total jump variation, while small jumps have unique information for predicting future returns, even compared to total jumps.

Panel C reports returns and alphas based on sorting on SRVSJ after controlling for SRVLJ. Both the equal- and value-weighted high-low spreads and alphas are statistically significant in this case, while this is not the case if stocks are first sorted by SRVSJ and then by SRVLJ, as shown in Panel D. More specifically, the high-low return is -25.38 bps (with a $t$-statistic of -6.61 ), for the value-weighted portfolio in Panel C, and is -3.77 bps with a $t$-statistics of -1.49 in Panel $\mathrm{D}$, for the value-weighted portfolio. This indicates that the predictable content in large jumps becomes negligible after controlling for small jumps.

Bollerslev et al. (2020) document that the negative association between realized skewness and one-week-ahead returns is reversed when controlling for the signed jump variation. To further investigate the relationship between skewness and different jump variation measures, we use double portfolio sorts to control for different effects that are associated with cross-sectional variation in future returns.

Panel A of Table 9 reports average returns and corresponding $t$-statistics for 25 portfolios sorted by SRVJ (signed jump variation), controlling for realized skewness (RSK). Inspection of the results in this table indicates that the negative association between SRVJ and future returns still exists, after controlling for RSK, indicating that there is unique predictive information contained in signed 
jump variation. Panel B in this table reports results for portfolios sorted first by SRVJ and then by RSK. The high-low spreads of the averaged portfolios are positive after controlling for SRVJ, confirming the results reported in Bollerslev et al. (2020).

Panel A of Table 10 contains results for portfolios sorted by SRVLJ (signed large jump variation) after controlling for RSK. As noted above, the negative association between SRVLJ and future returns is reversed after controlling for skewness. By contrast, this issue does not exist for portfolios sorted by SRVSJ (signed small jump variation) when controlling for skewness, as shown in Panel B of Table 10, indicating that signed small jump variation has unique information about future return premia. However, first accounting for skewness negates the usefulness that signed large jump variation has for predicting future returns. This finding serves as an important distinction between the predictive content of small and large jumps.

Finally, Table 11 contains results for portfolios sorted on RSK, after controlling for SRVLJ and SRVSJ, respectively. Inspection of the entries in this table indicates that the high-low spreads are negative, except in select value-weighted portfolio cases, when controlling for SRVSJ. This is not surprising since skewness captures information from both SRVLJ and SRVSJ, while the negative association between realized skewness and subsequent returns remains, when controlling for either SRVLJ or SRVSJ, in most cases. Of note is that this negative association disappears for some value-weighted portfolios, when controlling for SRVSJ, suggesting that signed small jump variation (especially for big firms) is the main driver of the signed total jump variation. These findings are consistent with the findings documented by Bollerslev and Todorov (2011) that S\&P 500 market portfolios tend to have symmetric jump tails (large jumps).

\subsection{Using Double Portfolio Sorts to Examine Stock-Level versus Industry-Level Predictability}

In this section, we carry out an additional set of double portfolio sort experiments, in which industry-based investing is compared with individual stock-based investing. Our earlier findings indicate that low signed jump variation investing (buying stocks with low signed jump variation and shorting stocks with high signed jump variation) can deliver significant risk-adjusted returns (this is a result also found by Bollerslev et al. (2020), for example). In order to examine whether this investment strategy relies on industry betting or stock selection within industries (or both), we form double-sorted portfolios based on industry-level and stock-level signed jump risk variation. In particular, each Tuesday we group stocks into 49 industries based on SIC codes. Industry-level signed jump risk is calculated as the value-weighted average of signed (large/small) jump variation measures for stocks within each industry. Thus, stocks in the same industry have the same industry signed (large/small) jump variation during a given week. Stock-level signed jump risk is calculated as outlined in the above. Double sorts are then used to investigate the selection effects at industry- and stock-level. Specifically, stocks are sorted into 25 portfolios based on industry- and stock-level signed (large/small) jump variation quintiles. With this particular variety of sorting, results are independent of the order in which stocks are sorted.

Figure 4 depicts the percentage of stocks in each portfolio (see Panel A), and the market capitalization in these portfolios (see Panel B). If industry-level selection and stock-level selection lead to different quintile portfolios (i.e., off-diagonal portfolios in the figures have non-zero membership), it is possible to separate these two effects using double-sorts. Specifically, there are different industryand stock-level effects. Both panels indicate this to be the case. 
Panel A: Average Distribution of Stocks Across Double-Sorted Portfolios

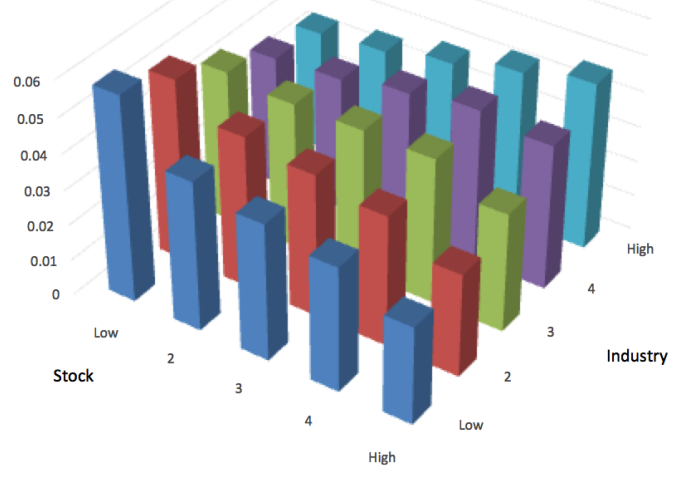

Panel B: Average Distribution of Market Capitalization Across Double-Sorted Portfolios

Figure 4. Distribution of Stocks in Portfolios Formed Based on Stocks' Signed Jump Variation (SRVJ) and Industry Signed Jump Variation. The vertical axis in Panels A and B measures time series average proportions of stocks and market capitalizations, across double-sorted portfolios.

Tables 12 and 13 report our empirical findings based on our double portfolio sort experiments. In particular, Table 12 reports results for sorting done on signed jump variation (SRVJ), while Table 13 reports results for sorting done on signed large jump variation (SRVLJ) and signed small jump variation (SRVSJ), respectively. Entries in the tables are mean returns and alphas, as in previous tables. However, in these tables we also report industry-level effects and stock-level effects. These are reported in the last two rows of entries in each panel of the tables. The first of these two rows, called "Industry-Level Effect" reports average high-low returns and alphas by averaging across quintiles in the high-low and alpha columns of the table (these are industry-level results). The second of these two rows, called "Stock-Level Effect" reports average high-low returns and alphas by averaging across quintiles in the high-low and alpha rows of the table (these are stock-level results). Summarizing, rows in these tables display portfolios formed by stocks in the same stock-level SRVJ, SRVLJ, or SRVSJ quintiles, while columns report results for portfolios formed by stocks in the same industry-level SRVJ, SRVLJ, or SRVSJ quintiles.

Turning to Table 12, notice, for example, that a strategy of buying stocks in the highest industry SRVJ quintile and selling stocks in the lowest industry SRVJ quintile generates an equal-weighted average return of 29.63 bps with a $t$-statistic of 5.66, and the corresponding value-weighted average return is 11.48 bps with a $t$-statistics of 2.23 (see Table 12). This finding is interesting, as it suggests that the negative association between SRVJ and future returns is reversed at the industry level. The equal-weighted average of the high-low row (i.e., the average stock-level effect) is $-45.28 \mathrm{bps}$ with a $t$-statistic of -11.39 and the alpha is -44.70 bps with a $t$-statistic of -11.50 , indicating that the stock-level effect is economically significant. At the stock-level, investors prefer stocks with high SRVJ, requiring lower returns under higher SRVJ, given that there is a large probability of extremely large positive jumps. By contrast, when sorting at the industry-level, investors are more interested in industry exposure with lower SRVJ, or in return distributions concentrated to the right. Lottery-like payoff exposure comes from individual stocks, not from industry bets. These results are mirrored in Table 13, where SRVLJ and SRVSJ are the sorting measures. However, average stock- and industry-level returns and alphas are much higher under SRVSJ sorting than under SRVLJ sorting. For example, buying stocks in the highest industry SRVLJ quintile and selling stocks in the lowest industry SRVLJ quintile generates an equal-weighted average return of 14.83 bps with a $t$-statistic of 3.77 under SRVLJ 
sorting (see Panel A of Table 13), versus an equal-weighted average return of $26.69 \mathrm{bps}$ with a $t$-statistic of 5.02 under SRVSJ sorting (see Panel B of Table 13).

\subsection{Firm-Level Fama-MacBeth Regressions}

Table 14 gathers results based on firm-level Fama-MacBeth regressions, which we run in order to investigate the return predictability associated with variation measures, when controlling for multiple firm specific characteristics. Regressions are carried out as follows. At the end of each Tuesday, we run the cross-sectional regression,

$$
r_{i, t+1}=\gamma_{0, t}+\sum_{j=1}^{K_{1}} \gamma_{j, t} X_{i, j, t}+\sum_{s=1}^{K_{2}} \phi_{s, t} Z_{i, s, t}+\epsilon_{i, t+1}, \quad t=1, \ldots, T,
$$

where $r_{i, t+1}$ denotes the stock return for firm $i$ in week $t+1, K_{1}$ is the number of potential variation measures, and $X_{i, j, t}$ denotes a relevant realized measure at the end of week $t$. In addition, there are $K_{2}$ variables measuring firm characteristics, which are denoted by $Z_{i, j, t}$ (see Section 3 for details). After estimating the cross-sectional regression coefficients on a weekly basis, we form the time series average of the resulting $T$ weekly $\widehat{\gamma}_{j, t}$ and $\widehat{\phi}_{s, t}$ values, in order to estimate the average risk premium associated with each risk measure. Specifically, we construct

$$
\widehat{\gamma}_{j}=\frac{1}{T} \sum_{t=1}^{T} \widehat{\gamma}_{j, t} \text { and } \widehat{\phi}_{s}=\frac{1}{T} \sum_{t=1}^{T} \widehat{\phi}_{s, t}, \text { for } j=1, \ldots, K_{1}, s=1, \ldots, K_{2} .
$$

Panel A of Table 14 reports results for regressions on various realized variation measures, without controlling for firm specific characteristics. Consistent with our results based on univariate sorting, signed jump variation (SRVJ) significantly negatively predicts cross-sectional variation, in these weekly returns regressions. Additionally, both signed small and large jump variation measures negatively predict future weekly returns. Finally, both small and large upside (downside) jump variation measures negatively (positively) predict subsequent weekly returns. However, when including measures that contain information from both small and large jump variation measures, as well as realized skewness, the negative association between skewness and future returns is reversed (see the results for the regressions labeled IX, XII, XV, XVI). In particular, skewness drives out signed large jump variation in regression XIII by reverting the negative association between the latter and future returns. If only small jumps are considered to be control variables, skewness still negatively predicts future returns. This again indicates that signed small jump variation has unique and significant information about future returns.

Panel B of Table 14 reports regression results for the same set of regressions in Panel A, but controlling for various firm specific characteristics, ranging from BETA to ILLIQ (see Table 1 for details). In these regressions, signed (small) jump variation is always significant. Additionally, skewness significantly negatively predicts future returns in regressions that only include small jump variation. This provides yet further evidence that signed small jump variation has unique and significant information about future returns, while large jumps have information in common with realized skewness.

\subsection{Pricing Distinctions between Small and Large Jumps}

The results in previous sections show that small and large jump variation measures contain different information, and thus have different predictive content. To further investigate whether the differences are driven by distinct economic factors, we provide empirical evidence on the inter-relationship between jumps and news. 


\subsubsection{Jumps and News Announcement}

We begin by examining the relationship between jumps and firm-level news announcements. In order to do this, we construct event windows using the approach of Bernard and Thomas (1989). We then plot the dynamics of SRVJ, SRVLJ, and SRVSJ around earnings announcements. In particular, following Livnat and Mendenhall (2006), the earning surprise (SUE) for each stock is defined as

$$
\operatorname{SUE} E_{j, t}=\frac{\left(X_{j, t}-E_{j, t}\right)}{P_{j, t}},
$$

where $E_{j, t}$ and $X_{j, t}$ denote the analysts' expectations and reported actual earnings per share, respectively. Here, $P_{j, t}$ is the price per share for stock $j$ at the end of quarter $t$. In a $[-12,12]$ week event window, where week zero denotes the earning announcement week, stocks are sorted into tertile portfolios by the value of SUE at the end of week zero. We then calculate the equal-weighted and value-weighted average of jump measures for each tertile portfolio at each week. Figure 5 displays various jump variation measures of portfolios with the most negative, median, and positive earning surprises. It turns out that large (both positive and negative) jump variation measures are higher during announcement weeks, regardless of news sentiment (i.e., regardless of whether SUE is positive or negative). However, positive large jump variation (RVLJP) is higher on days with the most positive earning surprises, and negative large jump variation (RVLJN) reaches its peak on days with the most negative earning surprises. In contrast, both small positive and negative jump variation measures (RVSJP and RVSJN) have lower magnitudes during announcement weeks. The size of the reduction associated with small positive jump variation (RVSJP) is larger on days with the most negative earning surprises, while small negative jump variation (RVSJN) decreases the most on days with the most positive surprises. For signed jump variation, jump magnitudes increase (relative to non-earnings-surprise weeks) on positive surprise days and decrease on negative surprising days. These results indicate that big news, regardless of sentiment, simultaneously leads to increases in the magnitude of large jump variation, and reductions in the level of small jump variation.

The other direction in which we investigate the linkage between news announcements and jump variation is based on an exploration of whether news announcements affect the frequency of occurrence of either small or large jumps. Table 16 reports the average percentage of firms exhibiting particular types of jumps on days with and without earning surprises. Specifically, on each announcement date, all stocks exhibiting earnings are sorted into tertile portfolios based on the absolute value of the earning surprise (SUE). The categories sorted on are denoted as "small", "medium", and "large", with tertiles calculated by appropriate sorting of the firms based on the absolute values of the firms' earnings surprise magnitudes. Then, within each tertile, the percentage of firms exhibiting a particular type of jump (averaged across all earnings surprise days) is calculated and reported. For these calculations, only days in which at least 3 firms report earning surprises and included in our sample. ${ }^{19}$ Turning to the results in the table, note, for example, that the entry 0.3042 in the sixth column of Panel A indicates that $30.42 \%$ of firms in the "small surprise" tertile portfolio recorded a large jump (measured by SRVLJ) on small surprise days, on average, across the entire daily sample. By contrast, 89.83\% of firms exhibit small jumps (measured by SRVSJ) on days with small surprises.

Two clear conclusions emerge upon examination of the results in this table. First, when the magnitude of earning surprises increases, the average percentage of firms with large jumps (SRVLJ) increases from $30.42 \%$ to $37.37 \%$. In particular, in Panel A, note that for the small tertile, the percentage of firms exhibiting large jumps (SRVLJ) is 30.42\%, while for the large tertile, the percentage is $37.37 \%$. By contrast, the percentage of firms with small jumps decreases as the relative magnitude of earnings surprises increases (i.e., the percentage of firms associated with SRVSJ decreases from $89.83 \%$ to

19 Results are virtually identical if we only include days in which at least 12 or 24 firms report earnings surprises. 
$88.29 \%$ ). This result indicates that "big news" is associated with an increase in the prevalence of large jumps. Second, the prevalence of jumps differs depending upon whether one tabulates results on earnings surprise days (Panel A) or on non-earnings surprise days (Panel B). For example, large news surprises are associated with large jumps for $31.07 \%$ of firms on non-announcement days (see Panel B) and $37.37 \%$ of firms on announcement days (Panel A). This result is consistent with event study finding that jump magnitudes are larger on announcement days than non-announcement days.

Panel A: Value-Weighted RVJP

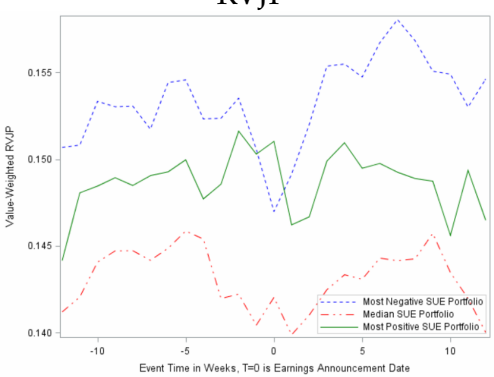

Panel D: Value-Weighted RVLJP

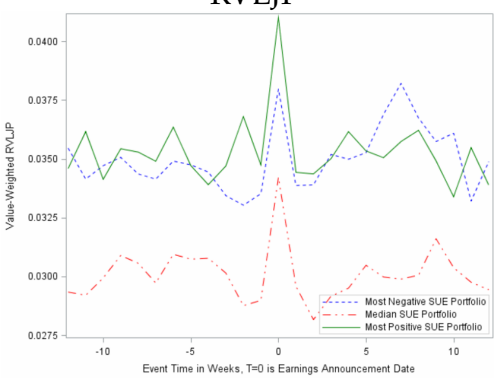

Panel G: Value-Weighted RVSJP

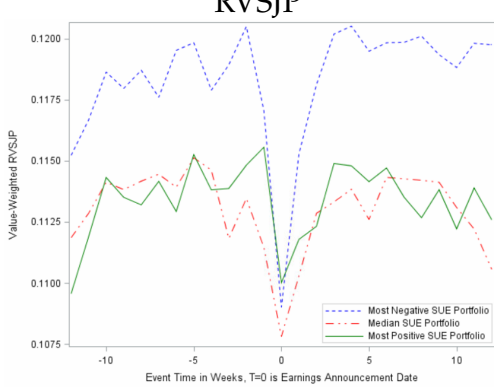

Panel B: Value-Weighted RVJN

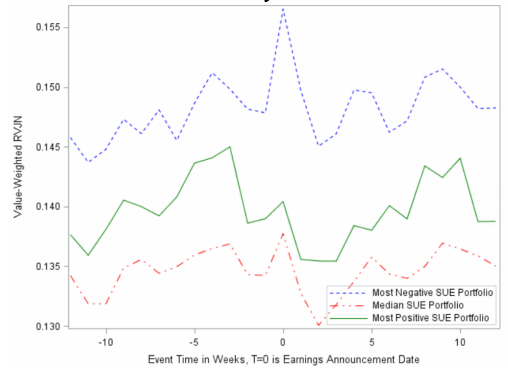

Panel E: Value-Weighted RVLJN

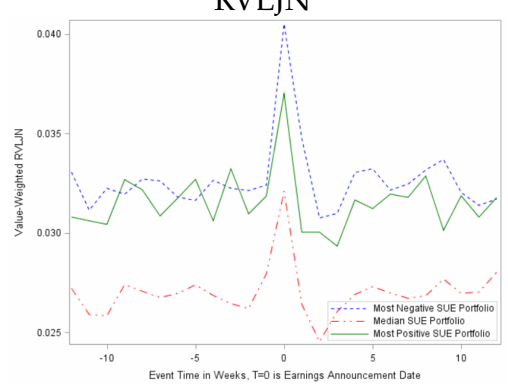

Panel H: Value-Weighted RVSJN

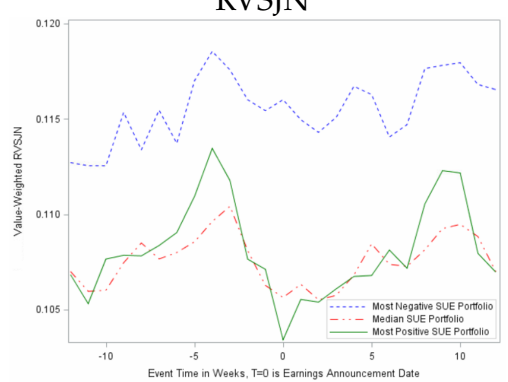

Panel C: Value-Weighted SRVJ

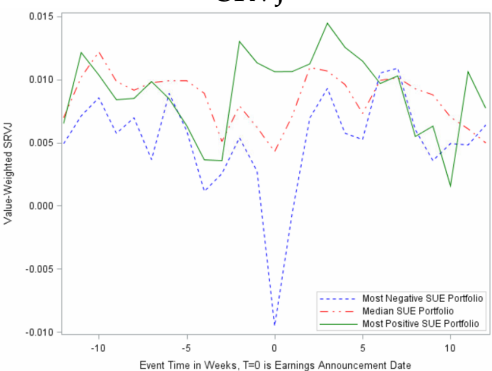

Panel F: Value-Weighted SRVLJ

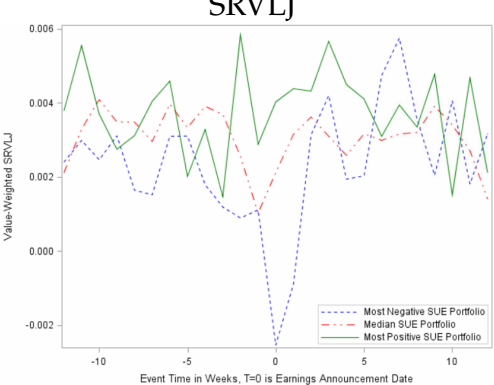

Panel I: Value-Weighted SRVSJ

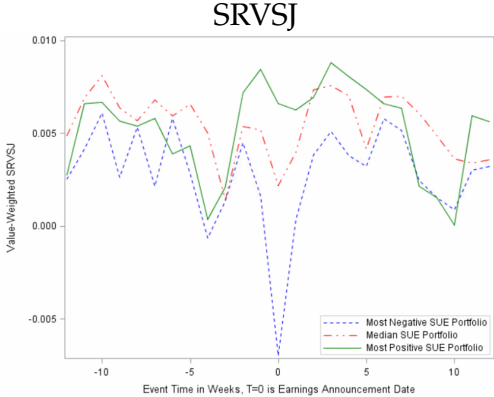

Figure 5. Jump Variation Measures Around Earnings Announcement. Panels A-I display value-weighted averages of various weekly jump variation measures in a $[-12,12]$ week window around earnings announcement. 
Table 16. Jumps Associated with (Absolute) Magnitude of Earning Surprises.

\begin{tabular}{lccccccccc}
\hline \multicolumn{7}{l}{ Panel A: Daily Average Percentage of Firms Exhibiting Various Types of Jumps, on Days Characterized by Earnings Surprises } \\
\hline A-SUE & RVJP & RVJN & SRVJ & RVLJP & RVLJN & SRVLJ & RVSJP & RVSJN & SRVSJ \\
\hline Small & 0.8099 & 0.8180 & 0.9849 & 0.1951 & 0.2004 & 0.3042 & 0.7233 & 0.7258 & 0.8983 \\
Medium & 0.8310 & 0.8232 & 0.9841 & 0.2216 & 0.2173 & 0.3289 & 0.7319 & 0.7232 & 0.8928 \\
Large & 0.8605 & 0.8621 & 0.9909 & 0.2618 & 0.2572 & 0.3737 & 0.7455 & 0.7488 & 0.8829 \\
\hline
\end{tabular}

Panel B: Daily Average Percentage of Firms Exhibiting Various Types of Jumps, on Days Characterized by No Earnings Surprises

\begin{tabular}{|c|c|c|c|c|c|c|c|c|}
\hline RVJP & RVJN & SRVJ & RVLJP & RVLJN & SRVLJ & RVSJP & RVSJN & SRVSJ \\
\hline 0.8836 & 0.8786 & 0.9884 & 0.2252 & 0.2220 & 0.3107 & 0.7941 & 0.7900 & 0.9095 \\
\hline \multicolumn{9}{|c|}{ Panel C: $t$-Statistics Associated with the Difference in Jump Size Percentages Between Portfolios } \\
\hline & & Difference & SRVJ & SRVLJ & SRVSJ & & & \\
\hline & & $\begin{array}{l}\text { Medium-Small } \\
\text { Large-Medium } \\
\text { Large-None }\end{array}$ & $\begin{array}{c}-0.55 \\
5.76 \\
3.54\end{array}$ & $\begin{array}{c}4.98 \\
9.02 \\
16.85\end{array}$ & $\begin{array}{l}-1.68 \\
-3.06 \\
-10.85\end{array}$ & & & \\
\hline
\end{tabular}

Notes: See notes to Table 1. Panels A and B of this table report daily average percentages of firms exhibiting various types of jumps, on days with (Panel A) and without (Panel B) earnings surprises. On earning announcement dates for which at least 3 stocks report earning, the "reporting" stocks are sorted into tertile portfolios (called "Small", "Medium", and "Large"), based on the absolute value of earning surprise (A-SUE), where SUE is defined in Equation (16). Thus, small, medium and large portfolios are only constructed on days for which at least 3 firms are characterized by an earnings surprise. Then, the percentage of firms exhibiting jumps in each of the three earnings surprise size categories is calculated, for various jump types (i.e., RVJP, RVJN, etc.) Finally, percentages are averages over all reporting days in the sample. Finally, various Newey-West $t$-statistics measuring the significance of the differences in jump size percentages for SRVJ, SRVLJ, and SRVSJ type jumps are reported in Panel C of the table. 
It is also worth noting that Panel $\mathrm{C}$ of Table 16 reports $t$-statistics that test whether the differences in percentages of jumps in different portfolios are significant. In this table, "None" refers to the case where percentages are calculated on non-earnings-announcement days. Thus, the fact that the "Large-None" $t$-statistic associated with SRVLJ is 16.85, indicates that the percentage of large jumps on "large-surprise" earnings announcement days is significantly greater than the percentage of large jumps on non-earnings-announcement days. This in turn implies that large jumps tend to occur on large-surprise earnings announcement days. On the other hand, the reverse is true in the case of small jumps. In particular, the "Large-None" $t$-statistic associated with SRVSJ is -10.85 , indicating that small jumps tend to occur on non-earnings-announcement days.

As discussed above, it is worth closing this section by stressing that the magnitudes of price movements depend on the magnitude of news (if they are related to news), and on the underlying process describing price evolution, and thus movements may manifest in the form of large/small jumps and/or price drifts. Clearly, the process associated with how firms and markets digest information is critical to understanding and quantifying what sorts of price movements ensue. For any given news, responses may differ in terms of upside or downside price drifts, or large/small jumps). In this sense, both small and large jumps may matter, as shown in our empirical results, in the cross-section.

\subsubsection{Systematic versus Idiosyncratic Risks}

To further explore the unique information embedded in either large or small jump variation measures, and examine their association with systematic and idiosyncratic risks, we identify the effect of diversification on both small and large jumps. In order to do this, we construct two alternative measures of SRVLJ and SRVSL. The ratio of these is plotted in Figure 6.

Method 1: For jump measures using this method, we simply construct SRVLJ and SRVSJ as done earlier in the paper. Specifically, we sort stocks into quintiles based on either weekly SRVLJ or SRVSJ. Then, we construct daily ratios of SRVLJ to SRVSJ for each individual stock in a given quintile. Finally, these ratios are aggregated, forming weekly measures of SRVLJ/SRVSJ. These measures are then used to form equal- or value-weighted ratios of SRVLJ to SRVSJ. These values are depicted in red (solid line) in Figure 6.

Method 2: For jump measures using this method, we start by constructing the same quintiles (based on weekly SRVLJ and SRVSJ) as done above. Then, we use the 5-min returns for each stock in a given quintile to construct 5-min aggregate portfolio returns for that quintile. We then construct daily jump measures using these portfolio returns (called SRVLJ and SRVSJ, and SRVLJ/SRVSJ), which are portfolio versions of the similar measures constructed using Method 1. Finally, daily measures are aggregated into weekly measures. These values are depicted in blue (dotted line) in Figure 6.

Comparing jump variation ratios constructed in these two different ways allows us to explore the importance of diversification when measuring jump variation. Turning to our findings, Figure 6 shows the time series of aggregated (Method 2) and weighted average (Method 1) jump variation measures for the first quintile portfolios. The fact that Method 1 (red line) is much smoother than Method 2 (blue line) means that the small jump component in the ratio of SRVLJ/SRVSJ remains much larger than in the other case. Thus, the obvious difference between aggregated and weighted averages of SRVLJ/SRVSJ indicates that small jump variation is more likely to be diversified away than large jump variation. This can be immediately seen upon examination of the plots in any of the four panels in the figure. Small jump variation is therefore more closely related to firm specific or idiosyncratic risks, while large jump variation is more likely to be systematic risks. ${ }^{20}$

20 See the Supplementary Appendix for plots of jump variation measures for the other quintile portfolios. 
Panel A: Equal-Weighted SRVLJ/SRVSJ (Stocks Sorted by SRVLJ)

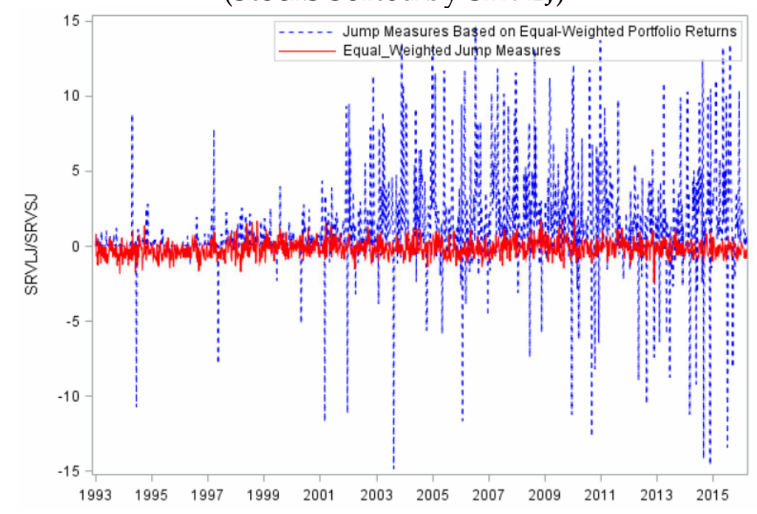

Panel C: Equal-Weighted SRVLJ/SRVSJ (Stocks Sorted by SRVSJ)

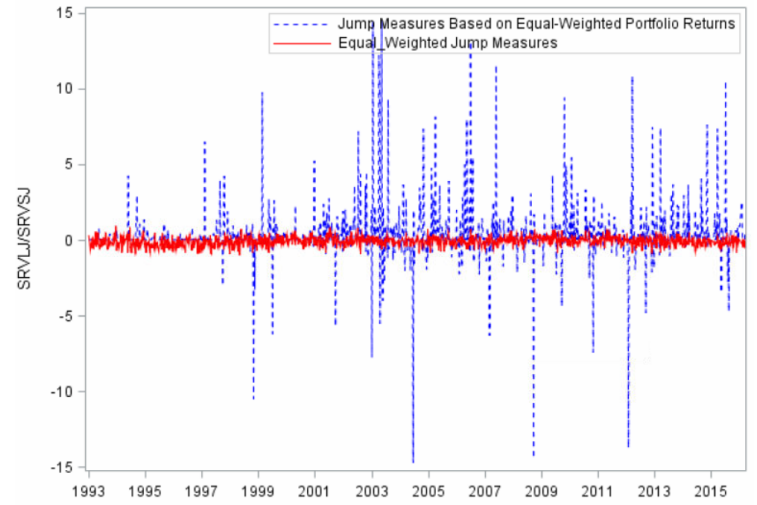

Panel B: Value-Weighted SRVLJ/SRVSJ (Stocks Sorted by SRVLJ)

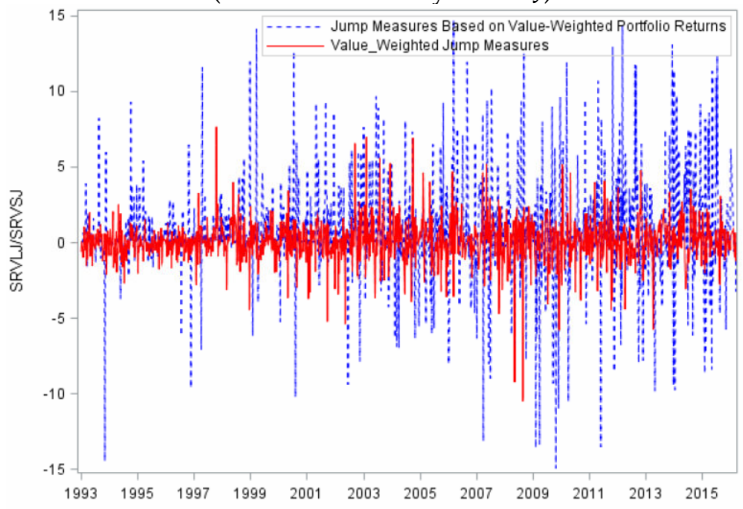

Panel D: Value-Weighted SRVLJ/SRVSJ (Stocks Sorted by SRVSJ)

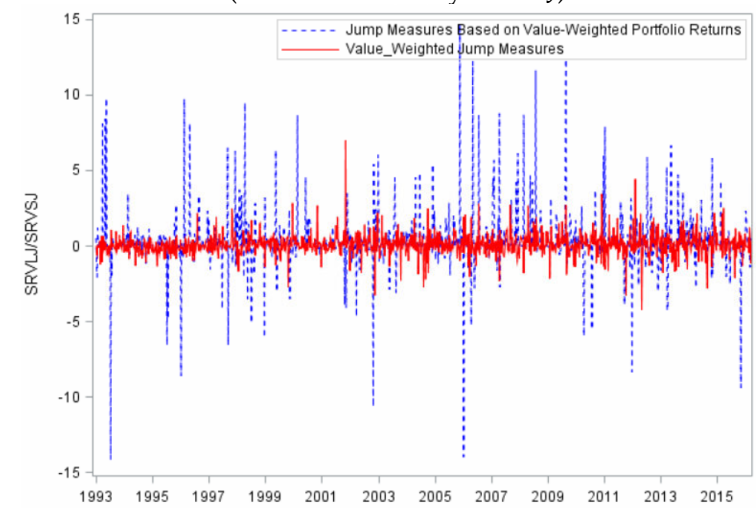

Figure 6. Aggregated and Weighted Average of Jump Variation Measures. Panels A-D display weekly aggregated and weighted averages of the ratio of SRVLJ to SRVSJ for 1st quintile stocks, sorted on SRVLJ and SRVSJ. Aggregated jump measures are depicted in blue (dotted line), and are constructed using 5-min portfolio returns. Weighted average jump measures are depicted in red (solid line) and are constructed using individual daily jump measures, and then aggregating to weekly. All calculation use jump truncation level $\gamma^{1}=4 \sqrt{\frac{1}{t} \widehat{I V}_{t}^{(i)}} \Delta_{n}^{0.49}$. For complete details, refer to Section 4.6.2.

Another way to explore the relationship between systematic and idiosyncratic risks is to carry out Fama-MacBeth type regressions where the dependent variable is one of our jump variation measures and the independent variables are firm characteristics. ${ }^{21}$ The results from several these sorts of regressions are reported in Table 17. Evidently, the firm characteristics always explain more of the dynamics associated with small jumps than with large jumps. This finding is supported by the fact that the adjusted $R^{2}$ is higher when the dependent variable is a small jump variation measure (compare the results of regressions I and II with III and IV). This again suggests that small jump variation is more likely to be associated with idiosyncratic risks. ${ }^{22}$

21 Specifically, our objective in this section is to discuss regressions of the form given in Equation (15), with the dependent variable replaced by various realized variables.

22 See the Supplementary Appendix for results from double-sorted portfolios that condition on various control variables. In these tables, it is noteworthy that when stocks are first sorted by a control variable (e.g., illiquidity, volatility, firm size and reversal), the SRVJ (SRVLJ and SRVSJ) effect is much higher within quintile portfolios with high illiquidity, high volatility, small firm size, and low reversal. This result suggests that all these control variables significantly contribute to the predictability of jump variation measures. This result provides additional confirmation to earlier findings reported in Bollerslev et al. (2020). 
Table 17. Fama-MacBeth Type Regressions Using Various Jump Variation Measures as Dependent Variable.

\begin{tabular}{|c|c|c|c|c|c|c|}
\hline & SRVLJ & SRVLJ & SRVSJ & SRVSJ & SRVJ & SRVJ \\
\hline & I & II & III & IV & $\mathbf{V}$ & VI \\
\hline Intercept & $\begin{array}{r}0.0080 \\
(5.81)\end{array}$ & $\begin{array}{r}0.0176 \\
(10.78)\end{array}$ & $\begin{array}{r}0.0034 \\
(2.59)\end{array}$ & $\begin{array}{r}0.0159 \\
(11.64)\end{array}$ & $\begin{array}{r}0.0115 \\
(4.63)\end{array}$ & $\begin{array}{l}0.0335 \\
(12.31)\end{array}$ \\
\hline RVOL & $\begin{array}{l}-0.0050 \\
(-11.02)\end{array}$ & $\begin{array}{r}0.0025 \\
(5.36)\end{array}$ & $\begin{array}{l}-0.0065 \\
(-15.37)\end{array}$ & $\begin{array}{r}0.0006 \\
(1.88)\end{array}$ & $\begin{array}{l}-0.0115 \\
(-14.81)\end{array}$ & $\begin{array}{r}0.0032 \\
(4.71)\end{array}$ \\
\hline Beta & & $\begin{array}{r}0.0014 \\
(4.40)\end{array}$ & & $\begin{array}{r}-0.0011 \\
(-3.76)\end{array}$ & & $\begin{array}{r}0.0003 \\
(0.79)\end{array}$ \\
\hline $\log ($ Size $)$ & $\begin{array}{r}0.0013 \\
(5.79)\end{array}$ & $\begin{array}{r}0.0003 \\
(1.62)\end{array}$ & $\begin{array}{r}0.0030 \\
(15.19)\end{array}$ & $\begin{array}{r}0.0017 \\
(9.21)\end{array}$ & $\begin{array}{r}0.0043 \\
(12.71)\end{array}$ & $\begin{array}{r}0.0020 \\
(6.67)\end{array}$ \\
\hline $\mathrm{BE} / \mathrm{ME}$ & & $\begin{array}{r}0.0007 \\
(4.11)\end{array}$ & & $\begin{array}{r}0.0003 \\
(2.72)\end{array}$ & & $\begin{array}{r}0.0010 \\
(4.58)\end{array}$ \\
\hline MOM & & $\begin{array}{r}0.0007 \\
(3.54)\end{array}$ & & $\begin{array}{r}0.0011 \\
(5.94)\end{array}$ & & $\begin{array}{r}0.0018 \\
(6.13)\end{array}$ \\
\hline REV & $\begin{array}{r}0.25166 \\
(61.05)\end{array}$ & $\begin{array}{r}0.1176 \\
(31.43)\end{array}$ & $\begin{array}{r}0.3172 \\
(65.55)\end{array}$ & $\begin{array}{c}0.1882 \\
(41.52)\end{array}$ & $\begin{array}{c}0.5688 \\
(69.66)\end{array}$ & $\begin{array}{r}0.3058 \\
(39.62)\end{array}$ \\
\hline IVOL & & $\begin{array}{l}-0.1424 \\
(-17.05)\end{array}$ & & $\begin{array}{l}-0.1880 \\
(-23.23)\end{array}$ & & $\begin{array}{l}-0.3303 \\
(-24.43)\end{array}$ \\
\hline CSK & & $\begin{array}{r}0.0142 \\
(19.14)\end{array}$ & & $\begin{array}{r}0.0206 \\
(28.33)\end{array}$ & & $\begin{array}{r}0.0349 \\
(26.38)\end{array}$ \\
\hline CKT & & $\begin{array}{r}-0.0008 \\
(-2.28)\end{array}$ & & $\begin{array}{r}-0.0008 \\
(-1.96)\end{array}$ & & $\begin{array}{r}-0.0015 \\
(-2.31)\end{array}$ \\
\hline MAX & & $\begin{array}{r}0.2456 \\
(43.77)\end{array}$ & & $\begin{array}{l}0.2248 \\
(25.28)\end{array}$ & & $\begin{array}{r}0.4704 \\
(35.84)\end{array}$ \\
\hline MIN & & $\begin{array}{l}0.5115 \\
(54.63)\end{array}$ & & $\begin{array}{l}0.4365 \\
(48.47)\end{array}$ & & $\begin{array}{l}0.9480 \\
(58.64)\end{array}$ \\
\hline ILLIQ & $\begin{array}{r}0.0020 \\
(6.33)\end{array}$ & $\begin{array}{r}0.0016 \\
(5.32)\end{array}$ & $\begin{array}{r}0.0032 \\
(18.60)\end{array}$ & $\begin{array}{l}0.0023 \\
(16.36)\end{array}$ & $\begin{array}{c}0.0052 \\
(12.20)\end{array}$ & $\begin{array}{c}0.0039 \\
(10.28)\end{array}$ \\
\hline Adjusted $R^{2}$ & 0.0322 & 0.0492 & 0.1070 & 0.1473 & 0.1049 & 0.1517 \\
\hline
\end{tabular}

Notes: See notes to Tables 1,5 and 14. This table reports results for cross-sectional Fama-MacBeth type regressions using various jump variation measures (listed across the first row of entries in the table) as dependent variables, and for various control variables (listed in the first column of the table). Thus, the regressions in this table mirror those reported in Table 14, with one difference. Specifically, the dependent variable in the regressions is either SRVLJ, SRVSJ, or SRVJ. Here, SRVLJ and SRVSJ are contructed using jump truncation level $\gamma^{2}=5 \sqrt{\frac{1}{t} \widehat{I V}_{t}^{(i)}} \Delta_{n}^{0.49}$.

\section{Concluding Remarks}

In this paper, we add to the literature that explores the relationship between equity returns and volatility. In particular, we focus on the strand of this literature that explores the data for evidence of asymmetry (non-linearity) in the return volatility trade-off. Following Bollerslev et al. (2020), we decompose realized variation into upside and downside semi-variances (good and bad volatilities). We then take the additional step of partitioning the semi-variances into small and large components. Within this context, we examine the marginal predictive content of small and large jump variation measures. We also examine the importance of earnings announcements for examining the linkages between small and large jumps and news.

We find that sorting on signed small jump variation leads to value-weighted high-low portfolios with greater average returns and alphas than when either signed total jump or signed large jump variation is sorted on. We also find that there is a threshold, beyond which large jump variation contains no marginal predictive ability, relative to that contained in small jump variation. Indeed, including large jump variation can actually be detrimental to predictive accuracy, as average returns and alphas for high-low portfolios actually decline when total variation is instead used in some of our prediction experiments. Analysis of returns and alphas based on industry double-sorts indicates that the benefit of small signed jump variation investing is driven by stock selection within an industry, 
rather than industry bets. Investors prefer stocks with a high probability of having positive jumps, but they also tend to overweight safer industries. Additionally, we find that the content of signed large jump variation is negligible when controlling for either signed total jump variation or realized skewness. By contrast, signed small jump variation has unique information for predicting future returns, even when controlling for total jump variation or realized skewness. Finally, we find that large jumps are closely associated with "big" news, or large earnings surprises, as might be expected. We also find that small jumps are more likely to be diversified away than large jumps, thus tend to be more closely associated with idiosyncratic risks, and are therefore more likely to be driven by liquidity conditions and trading activity. One reason for this is that while news-related information is embedded in large jump variation, the information is generally short-lived, and dissipates too quickly to provide marginal predictive content for subsequent weekly returns, in the particular cross-sectional setup that we use in our analysis.

Supplementary Materials: The Supplementary Materials available at http:/ /www.mdpi.com/2225-1146/8/2/19/s1.

Author Contributions: The authors contributed equally to this work. All authors have read and agreed to the published version of the manuscript.

Funding: This research received no external funding.

Acknowledgments: The authors are grateful to Mingmian Cheng, Yuan Liao, Xiye Yang and seminar participants at the 2019 SoFiE Conference, the 2019 Asia Meeting of the Econometric Society, and the 2019 Midwest Economics Association Conference for numerous useful comments. The authors are also grateful to the editor, Marc Paolella, and 3 referees for the many useful comments and suggestions that they made on earlier versions of this paper.

Conflicts of Interest: The authors declare no conflict of interest.

\section{References}

Ait-Sahalia, Yacine, and Jean Jacod. 2012. Analyzing the spectrum of asset returns: Jump and volatility components in high frequency data. Journal of Economic Literature 50: 1007-50. [CrossRef]

Amaya, Diego, Peter Christoffersen, Kris Jacobs, and Aurelio Vasquez. 2015. Does realized skewness predict the cross-section of equity returns? Journal of Financial Economics 118: 135-67. [CrossRef]

Amihud, Yakov. 2002. Illiquidity and stock returns: Cross-section and time-series effects. Journal of Financial Markets 5: 31-56. [CrossRef]

Andersen, Torben G., Tim Bollerslev, and Francis X. Diebold. 2007. Roughing it up: Including jump components in the measurement, modeling, and forecasting of return volatility. The Review of Economics and Statistics 89: 701-20. [CrossRef]

Andersen, Torben G., Tim Bollerslev, Francis X. Diebold, and Paul Labys. 2003. Modeling and forecasting realized volatility. Econometrica 71: 579-625. [CrossRef]

Ang, Andrew, Robert J. Hodrick, Yuhang Xing, and Xiaoyan Zhang. 2006. The cross-section of volatility and expected returns. The Journal of Finance 61: 259-99. [CrossRef]

Ang, Andrew, Robert J. Hodrick, Yuhang Xing, and Xiaoyan Zhang. 2009. High idiosyncratic volatility and low returns: International and further u.s. evidence. Journal of Financial Economics 91: 1-23. [CrossRef]

Bajgrowicz, Pierre, and Olivier Scaillet. 2012. Technical trading revisited: False discoveries, persistence tests, and transaction costs. Journal of Financial Economics 106: 473-91. [CrossRef]

Bajgrowicz, Pierre, Olivier Scaillet, and Adrien Treccani. 2016. Jumps in high-frequency data: Spurious detections, dynamics, and news. Management Science 62: 2198-217. [CrossRef]

Barndorff-Nielsen, Ole E., Svend Erik Graversen, Jean Jacod, and Neil Shephard. 2006. Limit theorems for bipower variation in financial econometrics. Econometric Theory 22: 677-719. [CrossRef]

Barndorff-Nielsen, Ole E., Silvia Kinnebrouk, and Neil Shephard. 2010. Measuring downside risk: Realised semivariance. In Volatility and Time Series Econometrics: Essays in Honor of Robert F. Engle. Edited by Tim Bollerslev, Jeffrey Russell and Mark Watson. Oxford: Oxford University Press, pp. 117-36.

Barndorff-Nielsen, Ole E., and Neil Shephard. 2004. Power and bipower variation with stochastic volatility and jumps. Journal of Financial Econometrics 2: 1-37. [CrossRef]

Barras, Laurent, Olivier Scaillet, and Russ Wermers. 2010. False discoveries in mutual fund performance: Measuring luck in estimated alphas. The Journal of Finance 65: 179-216. [CrossRef] 
Bernard, Victor L., and Jacob K. Thomas. 1989. Post-earnings-announcement drift: Delayed price response or risk premium? Journal of Accounting Research 27: 1-36. [CrossRef]

Bollerslev, Tim, Tzuo Hann Law, and George Tauchen. 2008. Risk, jumps, and diversification. Journal of Econometrics 144: 234-56. [CrossRef]

Bollerslev, Tim, Sophia Zhengzi Li, and Bingzhi Zhao. 2020. Good volatility, bad volatility, and the cross-section of stock returns. Journal of Financial and Quantitative Analysis 55: 751-81. [CrossRef]

Bollerslev, Tim, and Viktor Todorov. 2011. Estimation of jump tails. Econometrica 79: 1727-83.

Bollerslev, Tim, Viktor Todorov, and Lai Xu. 2015. Tail risk premia and return predictability. Journal of Financial Economics 118: 113-34. [CrossRef]

Campbell, John Y., and Tuomo Vuolteenaho. 2004. Bad beta, good beta. American Economic Review 94: 1249-75. [CrossRef]

Christensen, Kim, Roel C. A. Oomen, and Mark Podolskij. 2014. Fact or friction: Jumps at ultra high frequency. Journal of Financial Economics 114: 576-99. [CrossRef]

Duong, Diep, and Norman R. Swanson. 2011. Volatility in discrete and continuous-time models: A survey with new evidence on large and small jumps. In Missing Data Methods: Time-Series Methods and Applications. Edited by David M. Drukker. Advances in Econometrics, vol. 27B. Bingley: Emerald Press, pp. 179-233.

Duong, Diep, and Norman R. Swanson. 2015. Empirical evidence on the importance of aggregation, asymmetry, and jumps for volatility prediction. Journal of Econometrics 187: 606-21. [CrossRef]

Fang, Nengsheng, Wen Jiang, and Ronghua Luo. 2017. Realized semivariances and the variation of signed jumps in china's stock market. Emerging Markets Finance and Trade 53: 563-86. [CrossRef]

Feunou, Bruno, Mohammad R. Jahan-Parvar, and Romeo Tedongap. 2013. Modeling market downside volatility. Review of Finance 17: 443-81. [CrossRef]

Feunou, Bruno, Mohammad R. Jahan-Pravar, and Cédric Okou. 2018. Downside variance risk premium. Journal of Financial Econometrics 16: 341-83. [CrossRef]

Gu, Shihao, Bryan Kelly, and Dacheng Xiu. 2019. Empirical Asset Pricing via Machine Learning. Working Paper. Chicago: University of Chicago.

Guo, Hui, Kent Wang, and Hao Zhou. 2015. Good Jumps, Bad Jumps, and Conditional Equity Premium. Working Paper. Cincinnati: University of Cincinnati.

Jacod, Jean. 2008. Asymptotic properties of realized power variations and related functionals of semimartingales. Stochastic Processes and their Applications 118: 517-59. [CrossRef]

Jiang, George J., and Tong Yao. 2013. Stock price jumps and cross-sectional return predictability. Journal of Financial and Quantitative Analysis 48: 1519-44. [CrossRef]

Lee, Suzanne S., and Per A. Mykland. 2008. Jumps in financial markets: A new nonparametric test and jump dynamics. Review of Financial Studies 21: 2535-63. [CrossRef]

Lee, Suzanne S., and Per A. Mykland. 2012. Jumps in equilibrium prices and market microstructure noise. Journal of Econometrics 168: 396-406. [CrossRef]

Li, Jia, Viktor Todorov, George Tauchen, and Rui Chen. 2017. Mixed-scale jump regressions with bootstrap inference. Journal of Econometrics 201: 417-32. [CrossRef]

Livnat, Joshua, and Richard R. Mendenhall. 2006. Comparing the post-earnings announcement drift for surprises calculated from analyst and time series forecasts. Journal of Accounting Research 44: 177-205. [CrossRef]

Maheu, John M., and Thomas H. McCurdy. 2004. News arrival, jump dynamics, and volatility components for individual stock returns. Journal of Finance 59: 755-93. [CrossRef]

Mancini, Cecilia. 2009. Non-parametric threshold estimation for models with stochastic diffusion coefficient and jumps. Scandinavian Journal of Statistics 36: 270-96. [CrossRef]

Patton, Andrew J., and Kevin Sheppard. 2015. Good volatility, bad volatility: Signed jumps and the persistence of volatility. Review of Economics and Statistics 97: 683-97. [CrossRef]

Petersen, Mitchell A. 2009. Estimating standard errors in finance panel data sets: Comparing approaches. Review of Financial Studies 22: 435-80. [CrossRef]

Rossi, Alberto G., and Allan Timmermann. 2015. Modeling covariance risk in merton's icapm. Review of Financial Studies 28: 1428-61. [CrossRef]

Scaillet, Olivier, Adrien Treccani, and Christopher Trevisan. 2018. High-frequency jump analysis of the bitcoin market. Journal of Financial Econometrics. [CrossRef] 
Todorov, Viktor, and George Tauchen. 2010. Activity signature functions for high-frequency data analysis. Journal of Econometrics 154: 125-38. [CrossRef]

Woodward, George, and Heather M. Anderson. 2009. Does beta react to market conditions? Estimates of 'bull' and 'bear' betas using a nonlinear market model with an endogenous threshold parameter. Quantitative Finance 9: 913-24. [CrossRef]

Xiong, James X., Thomas M. Idzorek, and Roger G. Ibbotson. 2016. The economic value of forecasting left-tail risk. Journal of Portfolio Management 42: 114-23. [CrossRef]

Yan, Shu. 2011. Jump risk, stock returns, and slope of implied volatility smile. Journal of Financial Economics 99: 216-33. [CrossRef]

Yang, Hanxue and Juho Kanniainen. 2016. Jump and volatility dynamics for the s\&p 500: Evidence for infinite-activity jumps with non-affine volatility dynamics from stock and option markets. Review of Finance 21: 811-44.

(C) 2020 by the authors. Licensee MDPI, Basel, Switzerland. This article is an open access article distributed under the terms and conditions of the Creative Commons Attribution (CC BY) license (http://creativecommons.org/licenses/by/4.0/). 